\title{
Computational Study Investigating the Atmospheric Oxidation Mechanism and Kinetics of Dipropyl Thiosulfinate Initiated by OH Radicals, and the Fate of Propanethiyl Radical
}

\author{
Parandaman Arathala and Rabi A. Musah*
}

University at Albany-State University of New York, Department of Chemistry, 1400 Washington Avenue, Albany, NY 12222, USA

* Address correspondence to: rmusah@albany.edu

\section{Supporting Information}

This section contains: Tables S1-S10 display the calculated total electronic energies including zero-point energy corrections, optimized geometries in terms of their Z-matrices, their vibrational frequencies and rotational constants, relative energies, and imaginary frequencies of various TSs at the M06-2X levels of theory, rate constants, tunneling contributions, and branching ratios; Figure S1, S2 and S3 show optimized geometries of RCs, PCs, and products for DPTS $+\cdot \mathrm{OH}$, rate coefficient plot for the DPTS $+\cdot \mathrm{OH}$ association step to form pre-reactive complex (RC5), and PTR $+{ }^{3} \mathrm{O}_{2}$ reactions obtained at the M06-2X/6-311++G(3df,3pd) theory level. 
Table S1: Calculated total electronic energies for monomers, their complexes, and transition states (TSs) at the M06-2X, and CCSD(T) levels of theory. Zero point energy (ZPE) corrections are given at the M06-2X level.

\begin{tabular}{|c|c|c|c|}
\hline Species & M06-2X/6-311++G(3df,3pd) & ZPE (M06-2X) & CCSD(T)/cc-pVTZ \\
\hline DPTS & -1108.617450 & 0.196390 & -1107.207042 \\
\hline $\mathrm{OH}$ & -75.730338 & 0.008607 & -75.637723 \\
\hline DPTS••OH (RC1) & -1184.364259 & 0.207887 & -1182.859670 \\
\hline DPTS••OH (TS1) & -1184.350231 & 0.203297 & -1182.846082 \\
\hline DPTS••OH (PC1) & -1184.394994 & 0.207348 & -1182.888080 \\
\hline $\mathrm{CH}_{3}-\mathrm{CH}_{2}-\mathrm{C} \cdot \mathrm{H}-\mathrm{S}(\mathrm{O})-\mathrm{S}-\mathrm{CH}_{2}-\mathrm{CH}_{2}-\mathrm{CH}_{3}$ & -1107.954535 & 0.182562 & -1106.541861 \\
\hline $\mathrm{H}_{2} \mathrm{O}$ & -76.427007 & 0.021661 & -76.332161 \\
\hline DPTS••OH (RC2) & -1184.363402 & 0.209045 & -1182.855787 \\
\hline DPTS・・OH (TS2) & -1184.345511 & 0.202874 & -1182.841071 \\
\hline DPTS••OH (PC2) & -1184.389115 & 0.206802 & -1182.881379 \\
\hline DPTS $\bullet O H(T S 3)$ & -1184.351257 & 0.203484 & -1182.846648 \\
\hline DPTS••OH (PC3) & -1184.397735 & 0.206839 & -1182.890705 \\
\hline $\mathrm{CH}_{3}-\mathrm{C} \cdot \mathrm{H}-\mathrm{CH}_{2}-\mathrm{S}(\mathrm{O})-\mathrm{S}-\mathrm{CH}_{2}-\mathrm{CH}_{2}-\mathrm{CH}_{3}$ & -1107.955337 & 0.182031 & -1106.542315 \\
\hline DPTS・・OH (TS4) & -1184.348315 & 0.203894 & -1182.843600 \\
\hline DPTS••OH (PC4) & -1184.395303 & 0.206570 & -1182.886672 \\
\hline DPTS $\bullet O H(T S 5)$ & -1184.342609 & 0.203178 & -1182.837923 \\
\hline DPTS••OH (PC5) & -1184.388829 & 0.206639 & -1182.882138 \\
\hline DPTS••OH (TS6) & -1184.343776 & 0.203009 & -1182.839184 \\
\hline DPTS••OH (PC6) & -1184.380790 & 0.205690 & -1182.874182 \\
\hline $\mathrm{C} \cdot \mathrm{H}_{2}-\mathrm{CH}_{2}-\mathrm{CH}_{2}-\mathrm{S}(\mathrm{O})-\mathrm{S}-\mathrm{CH}_{2}-\mathrm{CH}_{2}-\mathrm{CH}_{3}$ & -1107.946443 & 0.181971 & -1106.534204 \\
\hline DPTS $\bullet \bullet O H(R C 3)$ & -1184.364579 & 0.208488 & -1182.859427 \\
\hline DPTS••OH (TS7) & -1184.353766 & 0.203611 & -1182.849629 \\
\hline DPTS••OH (PC7) & -1184.399405 & 0.206935 & -1182.890962 \\
\hline $\mathrm{CH}_{3}-\mathrm{CH}_{2}-\mathrm{CH}_{2}-\mathrm{S}(\mathrm{O})-\mathrm{S}-\mathrm{C} \cdot \mathrm{H}-\mathrm{CH}_{2}-\mathrm{CH}_{3}$ & -1107.957631 & 0.182492 & -1106.544533 \\
\hline DPTS••OH (TS8) & -1184.344853 & 0.203042 & -1182.840813 \\
\hline DPTS $\bullet O H(P C 8)$ & -1184.390625 & 0.205870 & -1182.882233 \\
\hline
\end{tabular}




\begin{tabular}{|c|c|c|c|}
\hline DPTS••OH (TS9) & -1184.345830 & 0.203423 & -1182.841162 \\
\hline DPTS••OH (PC9) & -1184.391204 & 0.206485 & -1182.883413 \\
\hline $\mathrm{CH}_{3}-\mathrm{CH}_{2}-\mathrm{CH}_{2}-\mathrm{S}(\mathrm{O})-\mathrm{S}-\mathrm{CH}_{2}-\mathrm{C} \cdot \mathrm{H}-\mathrm{CH}_{3}$ & -1107.954861 & 0.182326 & -1106.541133 \\
\hline DPTS••OH $(\mathrm{TS} 10)$ & -1184.347588 & 0.203666 & -1182.842799 \\
\hline DPTS••OH (PC10) & -1184.386443 & 0.205678 & -1182.877482 \\
\hline DPTS $\bullet O H(T S 11)$ & -1184.342459 & 0.203256 & -1182.837722 \\
\hline DPTS••OH $(\mathrm{PC} 11)$ & -1184.378847 & 0.205582 & -1182.872192 \\
\hline $\mathrm{CH}_{3}-\mathrm{CH}_{2}-\mathrm{CH}_{2}-\mathrm{S}(\mathrm{O})-\mathrm{S}-\mathrm{CH}_{2}-\mathrm{CH}_{2}-\mathrm{C} \cdot \mathrm{H}_{2}$ & -1107.946822 & 0.181852 & -1106.534494 \\
\hline DPTS••OH (TS12) & -1184.348962 & 0.204019 & -1182.844127 \\
\hline DPTS••OH (PC12) & -1184.387822 & 0.206469 & -1182.880356 \\
\hline DPTS••OH (RC4) & -1184.364607 & 0.208434 & -1182.859064 \\
\hline DPTS••OH (TS13) & -1184.354156 & 0.209341 & -1182.846606 \\
\hline DPTS••OH (PC13) & -1184.412409 & 0.210331 & -1182.904914 \\
\hline $\mathrm{CH}_{3}-\mathrm{CH}_{2}-\mathrm{CH}_{2}-\mathrm{S}-\mathrm{OH}$ & -592.509864 & 0.110130 & -591.7580308 \\
\hline $\mathrm{CH}_{3}-\mathrm{S} \bullet(=\mathrm{O})$ & -591.888288 & 0.098146 & -591.1337754 \\
\hline $\mathrm{DPTS} \bullet \mathrm{OH}(\mathrm{TS} 14)$ & -1184.359601 & 0.208861 & -1182.851642 \\
\hline DPTS••OH (RC5) & -1184.368705 & 0.211161 & -1182.863606 \\
\hline DPTS••OH (TS15) & -1184.365566 & 0.210177 & -1182.858400 \\
\hline DPTS••OH (PC15) & -1184.400847 & 0.210364 & -1182.892982 \\
\hline $\mathrm{CH}_{3} \mathrm{CH}_{2} \mathrm{CH}_{2}-\mathrm{S}(\mathrm{O})-\mathrm{OH}$ & -667.732695 & 0.114657 & -666.8778912 \\
\hline $\mathrm{CH}_{3}-\mathrm{CH}_{2}-\mathrm{CH}_{2}-\mathrm{S} \bullet$ & -516.657700 & 0.093456 & -516.0067086 \\
\hline 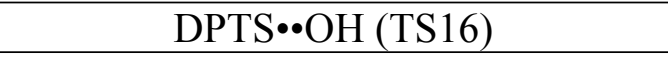 & -1184.361534 & 0.208392 & -1182.853072 \\
\hline DPTS••OH (PC16) & -1184.377455 & 0.206223 & -1182.867750 \\
\hline $\mathrm{CH}_{3} \mathrm{CH}_{2} \mathrm{CH}_{2} \mathrm{SS}(\mathrm{O}) \mathrm{OH}$ & -1065.931645 & 0.116304 & -1064.622253 \\
\hline $\mathrm{CH}_{3} \mathrm{CH}_{2} \mathrm{C} \cdot \mathrm{H}_{2}$ & -118.439332 & 0.088846 & -118.2412434 \\
\hline
\end{tabular}


Table S2. Relative energies $\left(\Delta \mathrm{E}_{0}{ }^{\ddagger}\right.$, $\left.\mathrm{kcal} \mathrm{mol}^{-1}\right)$ obtained for all the stationary points in the dipropyl thiosulfinate (DPTS) $+\cdot \cdot^{\mathrm{OH}}$ reactions at various levels of theory.

\begin{tabular}{|c|c|c|}
\hline Stationary point & M06-2X/6-311++G(3df,3pd) & $\begin{array}{c}\operatorname{CCSD}(T) / c c-p V T Z / / \\
\text { M06-2X/6-311++G(3df,3pd) }\end{array}$ \\
\hline $\mathrm{DPTS}+\cdot \mathrm{OH}$ & 0.0 & 0.0 \\
\hline $\mathrm{DPTS} \bullet \mathrm{OH}(\mathrm{RC} 1)$ & -8.5 & -7.5 \\
\hline DPTS••OH (TS1) & -2.6 & -1.9 \\
\hline DPTS••OH (PC1) & -28.1 & -25.7 \\
\hline $\mathrm{CH}_{3}-\mathrm{CH}_{2}-\mathrm{C} \cdot \mathrm{H}-\mathrm{S}(\mathrm{O})-\mathrm{S}-\mathrm{CH}_{2}-\mathrm{CH}_{2}-\mathrm{CH}_{3}+\mathrm{H}_{2} \mathrm{O}$ & -21.7 & -18.8 \\
\hline DPTS••OH (RC2) & -7.3 & -4.4 \\
\hline DPTS••OH (TS2) & 0.1 & 1.0 \\
\hline $\mathrm{DPTS} \bullet \mathrm{OH}(\mathrm{PC} 2)$ & -24.8 & -21.8 \\
\hline DPTS••OH (TS3) & -3.1 & -2.1 \\
\hline DPTS••OH (PC3) & -30.2 & -27.7 \\
\hline $\mathrm{CH}_{3}-\mathrm{C} \cdot \mathrm{H}-\mathrm{CH}_{2}-\mathrm{S}(\mathrm{O})-\mathrm{S}-\mathrm{CH}_{2}-\mathrm{CH}_{2}-\mathrm{CH}_{3}+\mathrm{H}_{2} \mathrm{O}$ & -22.5 & -19.5 \\
\hline DPTS••OH (TS4) & -1.0 & 0.0 \\
\hline $\mathrm{DPTS} \bullet \mathrm{OH}(\mathrm{PC} 4)$ & -28.8 & -25.3 \\
\hline DPTS••OH (TS5) & 2.1 & 3.2 \\
\hline DPTS $\bullet O H($ PC5) & -24.7 & -22.4 \\
\hline DPTS••OH (TS6) & 1.3 & 2.5 \\
\hline DPTS $\bullet$ OH (PC6) & -20.3 & -18.0 \\
\hline $\mathrm{C} \cdot \mathrm{H}_{2}-\mathrm{CH}_{2}-\mathrm{CH}_{2}-\mathrm{S}(\mathrm{O})-\mathrm{S}-\mathrm{CH}_{2}-\mathrm{CH}_{2}-\mathrm{CH}_{3}+\mathrm{H}_{2} \mathrm{O}$ & -17.0 & -14.4 \\
\hline DPTS••OH (RC3) & -8.3 & -7.0 \\
\hline DPTS••OH (TS7) & -4.6 & -3.9 \\
\hline DPTS••OH (PC7) & -31.2 & -27.8 \\
\hline $\mathrm{CH}_{3}-\mathrm{CH}_{2}-\mathrm{CH}_{2}-\mathrm{S}(\mathrm{O})-\mathrm{S}-\mathrm{C} \cdot \mathrm{H}-\mathrm{CH}_{2}-\mathrm{CH}_{3}+\mathrm{H}_{2} \mathrm{O}$ & -23.7 & -20.6 \\
\hline DPTS••OH (TS8) & 0.6 & 1.3 \\
\hline DPTS••OH (PC8) & -26.3 & -23.0 \\
\hline
\end{tabular}




\begin{tabular}{|c|c|c|}
\hline DPTS••OH (TS9) & 0.2 & 1.3 \\
\hline DPTS••OH (PC9) & -26.3 & -23.3 \\
\hline $\mathrm{CH}_{3}-\mathrm{CH}_{2}-\mathrm{CH}_{2}-\mathrm{S}(\mathrm{O})-\mathrm{S}-\mathrm{CH}_{2}-\mathrm{C} \cdot \mathrm{H}-\mathrm{CH}_{3}+\mathrm{H}_{2} \mathrm{O}$ & -22.0 & -18.5 \\
\hline DPTS $\bullet O H ~(T S 10)$ & -0.7 & 0.4 \\
\hline DPTS••OH (PC10) & -23.8 & -20.1 \\
\hline $\mathrm{DPTS} \bullet \bullet \mathrm{OH}(\mathrm{TS} 11)$ & 2.3 & 3.3 \\
\hline $\mathrm{DPTS} \bullet \bullet \mathrm{OH}(\mathrm{PC} 11)$ & -19.1 & -16.8 \\
\hline $\mathrm{CH}_{3}-\mathrm{CH}_{2}-\mathrm{CH}_{2}-\mathrm{S}(\mathrm{O})-\mathrm{S}-\mathrm{CH}_{2}-\mathrm{CH}_{2}-\mathrm{C} \cdot \mathrm{H}_{2}+\mathrm{H}_{2} \mathrm{O}$ & -17.3 & -14.7 \\
\hline DPTS••OH (TS12) & -8.3 & -0.2 \\
\hline DPTS••OH (PC12) & -24.2 & -21.4 \\
\hline DPTS••OH (RC4) & -8.4 & -6.8 \\
\hline $\mathrm{DPTS} \bullet \mathrm{OH}(\mathrm{TS} 13)$ & -1.3 & 1.6 \\
\hline DPTS • OH (PC13) & -37.2 & -34.4 \\
\hline $\mathrm{CH}_{3}-\mathrm{CH}_{2}-\mathrm{CH}_{2}-\mathrm{S}-\mathrm{OH}+\mathrm{CH}_{3}-\mathrm{S} \bullet(=\mathrm{O})$ & -29.5 & -27.5 \\
\hline DPTS••OH (TS14) & -5.0 & -1.9 \\
\hline DPTS••OH (RC5) & -9.3 & -8.0 \\
\hline $\mathrm{DPTS} \bullet \mathrm{OH}(\mathrm{TS} 15)$ & -7.9 & -5.4 \\
\hline DPTS••OH (PC15) & -29.8 & -26.9 \\
\hline $\mathrm{CH}_{3} \mathrm{CH}_{2} \mathrm{CH}_{2}-\mathrm{S}(\mathrm{O})-\mathrm{OH}+\mathrm{CH}_{3}-\mathrm{CH}_{2}-\mathrm{CH}_{2}-\mathrm{S} \bullet$ & -24.8 & -23.0 \\
\hline DPTS••OH (TS16) & -6.5 & -3.1 \\
\hline DPTS••OH (PC16) & -17.8 & -13.7 \\
\hline $\mathrm{CH}_{3} \mathrm{CH}_{2} \mathrm{CH}_{2} \mathrm{SS}(\mathrm{O}) \mathrm{OH}+\mathrm{CH}_{3} \mathrm{CH}_{2} \mathrm{C} \cdot \mathrm{H}_{2}$ & -14.5 & -11.7 \\
\hline
\end{tabular}


Table S3: Imaginary frequencies of all transition states at the M06-2X/6-311++G(3df,3pd) level of theory.

\begin{tabular}{|c|c|}
\hline Transition State & M06-2X/6-311++G(3df,3pd) \\
\hline DPTS••OH (TS1) & 1408 \\
\hline DPTS••OH (TS2) & 994 \\
\hline DPTS ••OH (TS3) & 1107 \\
\hline DPTS••OH (TS4) & 883 \\
\hline DPTS ••OH (TS5) & 828 \\
\hline DPTS••OH (TS6) & 757 \\
\hline DPTS ••OH (TS7) & 1084 \\
\hline DPTS••OH (TS8) & 641 \\
\hline DPTS••OH (TS9) & 796 \\
\hline DPTS••OH (TS10) & 709 \\
\hline DPTS••OH (TS11) & 777 \\
\hline 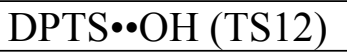 & 1219 \\
\hline DPTS••OH (TS13) & 560 \\
\hline DPTS••OH (TS14) & 499 \\
\hline DPTS••OH (TS15) & 117 \\
\hline DPTS••OH (TS16) & 224 \\
\hline
\end{tabular}

Table S4: Calculated rotational constants (GHz) for monomers, their complexes, and transition states (TS) at the M06-2X level of theory.

\begin{tabular}{|c|c|c|c|}
\hline Species & $\mathbf{A}$ & B & C \\
\hline DPTS & 3.01 & 0.47 & 0.45 \\
\hline $\mathrm{OH}$ & 565.85 & & \\
\hline $\mathrm{DPTS} \bullet \bullet \mathrm{OH}(\mathrm{RC} 1)$ & 1.96 & 0.42 & 0.40 \\
\hline DPTS••OH (TS1) & 1.91 & 0.41 & 0.39 \\
\hline DPTS $\bullet O H(\mathrm{PC} 1)$ & 1.81 & 0.44 & 0.42 \\
\hline $\mathrm{CH}_{3}-\mathrm{CH}_{2}-\mathrm{C} \cdot \mathrm{H}-\mathrm{S}(\mathrm{O})-\mathrm{S}-\mathrm{CH}_{2}-\mathrm{CH}_{2}-\mathrm{CH}_{3}$ & 2.73 & 0.56 & 0.52 \\
\hline $\mathrm{H}_{2} \mathrm{O}$ & 830.74 & 433.04 & 284.66 \\
\hline DPTS $\bullet \circ \mathrm{OH}(\mathrm{RC} 2)$ & 1.94 & 0.46 & 0.43 \\
\hline DPTS••OH (TS2) & 1.64 & 0.45 & 0.41 \\
\hline DPTS••OH (PC2) & 1.16 & 0.60 & 0.47 \\
\hline DPTS ••OH (TS3) & 2.00 & 0.40 & 0.37 \\
\hline DPTS••OH (PC3) & 1.74 & 0.45 & 0.42 \\
\hline $\mathrm{CH}_{3}-\mathrm{C} \cdot \mathrm{H}-\mathrm{CH}_{2}-\mathrm{S}(\mathrm{O})-\mathrm{S}-\mathrm{CH}_{2}-\mathrm{CH}_{2}-\mathrm{CH}_{3}$ & 2.85 & 0.52 & 0.49 \\
\hline DPTS $\bullet$ OH $(\mathrm{TS} 4)$ & 1.79 & 0.43 & 0.38 \\
\hline DPTS••OH (PC4) & 1.44 & 0.45 & 0.40 \\
\hline DPTS••OH (TS5) & 2.13 & 0.35 & 0.33 \\
\hline DPTS••OH (PC5) & 1.84 & 0.41 & 0.39 \\
\hline
\end{tabular}




\begin{tabular}{|c|c|c|c|}
\hline DPTS••OH (TS6) & 1.83 & 0.36 & 0.33 \\
\hline DPTS••OH (PC6) & 1.59 & 0.39 & 0.36 \\
\hline $\mathrm{C} \cdot \mathrm{H}_{2}-\mathrm{CH}_{2}-\mathrm{CH}_{2}-\mathrm{S}(\mathrm{O})-\mathrm{S}-\mathrm{CH}_{2}-\mathrm{CH}_{2}-\mathrm{CH}_{3}$ & 2.95 & 0.48 & 0.46 \\
\hline DPTS••OH (RC3) & 1.78 & 0.45 & 0.39 \\
\hline DPTS••OH (TS7) & 1.90 & 0.43 & 0.40 \\
\hline DPTS••OH (PC7) & 1.80 & 0.43 & 0.39 \\
\hline $\mathrm{CH}_{3}-\mathrm{CH}_{2}-\mathrm{CH}_{2}-\mathrm{S}(\mathrm{O})-\mathrm{S}-\mathrm{C} \cdot \mathrm{H}-\mathrm{CH}_{2}-\mathrm{CH}_{3}$ & 3.07 & 0.48 & 0.45 \\
\hline DPTS••OH (TS8) & 1.95 & 0.37 & 0.35 \\
\hline DPTS••OH (PC8) & 1.97 & 0.35 & 0.33 \\
\hline DPTS••OH (TS9) & 1.76 & 0.42 & 0.39 \\
\hline DPTS••OH (PC9) & 1.36 & 0.53 & 0.50 \\
\hline $\mathrm{CH}_{3}-\mathrm{CH}_{2}-\mathrm{CH}_{2}-\mathrm{S}(\mathrm{O})-\mathrm{S}-\mathrm{CH}_{2}-\mathrm{C} \cdot \mathrm{H}-\mathrm{CH}_{3}$ & 1.76 & 0.42 & 0.40 \\
\hline DPTS $\bullet$ OH $($ TS10) & 1.80 & 0.41 & 0.37 \\
\hline $\mathrm{DPTS} \bullet \mathrm{OH}(\mathrm{PC} 10)$ & 1.80 & 0.39 & 0.35 \\
\hline DPTS $\bullet O H(T S 11)$ & 1.80 & 0.33 & 0.31 \\
\hline $\mathrm{DPTS} \bullet \bullet \mathrm{OH}(\mathrm{PC} 11)$ & 1.50 & 0.35 & 0.32 \\
\hline $\mathrm{CH}_{3}-\mathrm{CH}_{2}-\mathrm{CH}_{2}-\mathrm{S}(\mathrm{O})-\mathrm{S}-\mathrm{CH}_{2}-\mathrm{CH}_{2}-\mathrm{C} \cdot \mathrm{H}_{2}$ & 3.01 & 0.49 & 0.46 \\
\hline DPTS $\bullet \cdot O H(T S 12)$ & 1.69 & 0.47 & 0.39 \\
\hline DPTS••OH (PC12) & 1.71 & 0.46 & 0.39 \\
\hline DPTS••OH (RC4) & 1.70 & 0.45 & 0.42 \\
\hline DPTS $\bullet O H(T S 13)$ & 1.91 & 0.43 & 0.41 \\
\hline DPTS••OH (PC13) & 1.69 & 0.41 & 0.37 \\
\hline $\mathrm{CH}_{3}-\mathrm{CH}_{2}-\mathrm{CH}_{2}-\mathrm{S}-\mathrm{OH}$ & 9.61 & 1.91 & 1.72 \\
\hline $\mathrm{CH}_{3}-\mathrm{S} \bullet(=\mathrm{O})$ & 11.28 & 1.95 & 1.78 \\
\hline DPTS ••OH (TS14) & 2.08 & 0.45 & 0.42 \\
\hline DPTS••OH (PC14) & 1.07 & 0.56 & 0.43 \\
\hline DPTS••OH (RC5) & 1.76 & 0.48 & 0.43 \\
\hline DPTS $\bullet \circ H(T S 15)$ & 2.07 & 0.45 & 0.44 \\
\hline DPTS••OH (PC15) & 1.01 & 0.56 & 0.48 \\
\hline $\mathrm{CH}_{3} \mathrm{CH}_{2} \mathrm{CH}_{2}-\mathrm{S}(\mathrm{O})-\mathrm{OH}$ & 5.65 & 1.69 & 1.55 \\
\hline $\mathrm{CH}_{3}-\mathrm{CH}_{2}-\mathrm{CH}_{2}-\mathrm{S} \bullet$ & 26.80 & 2.41 & 2.31 \\
\hline DPTS••OH (TS16) & 2.10 & 0.44 & 0.42 \\
\hline DPTS••OH (PC16) & 1.64 & 0.40 & 0.37 \\
\hline $\mathrm{CH}_{3} \mathrm{CH}_{2} \mathrm{CH}_{2} \mathrm{SS}(\mathrm{O}) \mathrm{OH}$ & 3.65 & 1.02 & 0.87 \\
\hline $\mathrm{CH}_{3} \mathrm{CH}_{2} \mathrm{C} \cdot \mathrm{H}_{2}$ & 31.17 & 8.99 & 8.05 \\
\hline
\end{tabular}


Table S5: Calculated positive frequencies $\left(\mathrm{cm}^{-1}\right)$ for monomers, their complexes, and transition states (TSs) at the M06-2X levels of theory.

\begin{tabular}{|c|c|c|c|c|c|}
\hline \multirow{2}{*}{$\begin{array}{l}\text { Species } \\
\text { DPTS }\end{array}$} & \multicolumn{5}{|c|}{ Frequencies } \\
\hline & 18 & 52 & 79 & 87 & 95 \\
\hline & 116 & 182 & 130 & 239 & 248 \\
\hline & 259 & 299 & 344 & 380 & 419 \\
\hline & 504 & 725 & 734 & 751 & 754 \\
\hline & 851 & 864 & 916 & 920 & 1059 \\
\hline & 1059 & 1074 & 1080 & 1115 & 1121 \\
\hline & 1163 & 1239 & 1251 & 1260 & 1271 \\
\hline & 1324 & 1331 & 1371 & 1377 & 1413 \\
\hline & 1416 & 1454 & 1472 & 1495 & 1500 \\
\hline & 1502 & 1503 & 1510 & 1513 & 3041 \\
\hline & 3044 & 3052 & 3060 & 3064 & 3071 \\
\hline & 3088 & 3101 & 3110 & 3121 & 3122 \\
\hline & 3123 & 3124 & 3143 & & \\
\hline •OH & \multicolumn{5}{|l|}{3778} \\
\hline \multirow[t]{14}{*}{ DPTS••OH (RC1) } & 21 & 26 & 53 & 63 & 70 \\
\hline & 99 & 116 & 148 & 179 & 205 \\
\hline & 234 & 246 & 250 & 267 & 311 \\
\hline & 344 & 382 & 427 & 492 & 521 \\
\hline & 639 & 716 & 728 & 752 & 754 \\
\hline & 852 & 868 & 918 & 924 & 1059 \\
\hline & 1060 & 1078 & 1080 & 1116 & 1122 \\
\hline & 1124 & 1238 & 1249 & 1265 & 1270 \\
\hline & 1326 & 1332 & 1372 & 1377 & 1415 \\
\hline & 1417 & 1453 & 1471 & 1494 & 1494 \\
\hline & 1503 & 1505 & 1509 & 1510 & 3044 \\
\hline & 3044 & 3054 & 3054 & 3064 & 3072 \\
\hline & 3089 & 3096 & 3111 & 3121 & 3123 \\
\hline & 3128 & 3129 & 3143 & 3537 & \\
\hline \multirow[t]{9}{*}{ DPTS••OH (TS1) } & -1408 & 19 & 47 & 52 & 75 \\
\hline & 89 & 118 & 125 & 178 & 185 \\
\hline & 231 & 246 & 255 & 266 & 308 \\
\hline & 349 & 383 & 432 & 462 & 516 \\
\hline & 642 & 719 & 729 & 752 & 805 \\
\hline & 849 & 907 & 918 & 924 & 956 \\
\hline & 1057 & 1060 & 1080 & 1106 & 1119 \\
\hline & 1122 & 1138 & 1245 & 1249 & 1271 \\
\hline & 1304 & 1325 & 1371 & 1374 & 1414 \\
\hline
\end{tabular}




\begin{tabular}{|c|c|c|c|c|c|}
\hline & $\begin{array}{l}1416 \\
1495 \\
3041 \\
3088 \\
3125\end{array}$ & $\begin{array}{l}1474 \\
1504 \\
3042 \\
3099 \\
3126\end{array}$ & $\begin{array}{l}1481 \\
1505 \\
3050 \\
3110 \\
3138\end{array}$ & $\begin{array}{c}1491 \\
1510 \\
3053 \\
3117 \\
3687\end{array}$ & $\begin{array}{l}1495 \\
1513 \\
3065 \\
3120\end{array}$ \\
\hline DPTS••OH (PC1) & $\begin{array}{l}23 \\
101 \\
230 \\
309 \\
476 \\
752 \\
1037 \\
1144 \\
1287 \\
1412 \\
1504 \\
3043 \\
3110 \\
3147\end{array}$ & $\begin{array}{l}29 \\
116 \\
241 \\
324 \\
542 \\
796 \\
1055 \\
1151 \\
1327 \\
1472 \\
1508 \\
3048 \\
3112 \\
3204\end{array}$ & $\begin{array}{l}64 \\
151 \\
255 \\
349 \\
572 \\
853 \\
1060 \\
1243 \\
1371 \\
1473 \\
1511 \\
3055 \\
3123 \\
3759\end{array}$ & $\begin{array}{l}81 \\
174 \\
262 \\
381 \\
691 \\
916 \\
1079 \\
1249 \\
1372 \\
1495 \\
1652 \\
3068 \\
3125 \\
3943\end{array}$ & $\begin{array}{l}85 \\
197 \\
275 \\
448 \\
729 \\
942 \\
1120 \\
1269 \\
1402 \\
1496 \\
3009 \\
3089 \\
3125\end{array}$ \\
\hline $\mathrm{CH}_{3} \mathrm{CH}_{2} \mathrm{C} \cdot \mathrm{H}-\mathrm{S}(\mathrm{O})-\mathrm{S}-\mathrm{C}_{3} \mathrm{H}_{7}$ & $\begin{array}{l}16 \\
159 \\
267 \\
478 \\
806 \\
1060 \\
1171 \\
1327 \\
1474 \\
1510 \\
3060 \\
3124\end{array}$ & $\begin{array}{l}48 \\
207 \\
302 \\
547 \\
853 \\
1061 \\
1249 \\
1337 \\
1478 \\
1511 \\
3066 \\
3125\end{array}$ & $\begin{array}{l}57 \\
226 \\
334 \\
663 \\
919 \\
1081 \\
1258 \\
1372 \\
1495 \\
3045 \\
3092 \\
3137\end{array}$ & $\begin{array}{l}72 \\
240 \\
379 \\
728 \\
923 \\
1122 \\
1268 \\
1404 \\
1497 \\
3049 \\
3108 \\
3145\end{array}$ & $\begin{array}{l}80 \\
252 \\
420 \\
749 \\
1031 \\
1125 \\
1315 \\
1416 \\
1506 \\
3054 \\
3112 \\
3220\end{array}$ \\
\hline $\mathrm{H}_{2} \mathrm{O}$ & 1619 & 3893 & 3996 & & \\
\hline DPTS••OH (RC2) & $\begin{array}{l}40 \\
117 \\
233 \\
295 \\
728 \\
855 \\
1061 \\
1169 \\
1329 \\
1417\end{array}$ & $\begin{array}{l}49 \\
135 \\
247 \\
336 \\
735 \\
870 \\
1073 \\
1239 \\
1332 \\
1454\end{array}$ & $\begin{array}{l}70 \\
152 \\
251 \\
380 \\
740 \\
918 \\
1082 \\
1244 \\
1374 \\
1470\end{array}$ & $\begin{array}{l}89 \\
188 \\
260 \\
416 \\
745 \\
921 \\
1113 \\
1253 \\
1375 \\
1488\end{array}$ & $\begin{array}{l}94 \\
218 \\
280 \\
498 \\
752 \\
1057 \\
1116 \\
1266 \\
1415 \\
1499\end{array}$ \\
\hline
\end{tabular}




\begin{tabular}{|c|c|c|c|c|c|}
\hline & $\begin{array}{l}1504 \\
3058 \\
3106 \\
3139\end{array}$ & $\begin{array}{l}1505 \\
3061 \\
3110 \\
3142\end{array}$ & $\begin{array}{l}1510 \\
3064 \\
3119 \\
3156\end{array}$ & $\begin{array}{l}1512 \\
3079 \\
3132 \\
3845\end{array}$ & $\begin{array}{l}3055 \\
3089 \\
3137\end{array}$ \\
\hline DPTS••OH (TS2) & $\begin{array}{l}14 \\
99 \\
248 \\
336 \\
730 \\
857 \\
1060 \\
1164 \\
1301 \\
1416 \\
1505 \\
3058 \\
3099 \\
3138\end{array}$ & $\begin{array}{l}41 \\
112 \\
252 \\
375 \\
736 \\
918 \\
1083 \\
1239 \\
1327 \\
1418 \\
1505 \\
3064 \\
3110 \\
3145\end{array}$ & $\begin{array}{c}62 \\
122 \\
259 \\
418 \\
749 \\
939 \\
1099 \\
1251 \\
1332 \\
1474 \\
1509 \\
3065 \\
3123 \\
3750\end{array}$ & $\begin{array}{l}79 \\
188 \\
279 \\
499 \\
760 \\
995 \\
1120 \\
1262 \\
1373 \\
1485 \\
1511 \\
3076 \\
3134\end{array}$ & $\begin{array}{c}85 \\
241 \\
310 \\
692 \\
853 \\
1059 \\
1123 \\
1271 \\
1377 \\
1495 \\
3055 \\
3094 \\
3136\end{array}$ \\
\hline DPTS••OH (PC2) & $\begin{array}{l}19 \\
99 \\
177 \\
291 \\
420 \\
750 \\
1035 \\
1151 \\
1299 \\
1417 \\
1505 \\
3052 \\
3111 \\
3149\end{array}$ & $\begin{array}{l}41 \\
117 \\
216 \\
306 \\
447 \\
827 \\
1054 \\
1171 \\
1327 \\
1473 \\
1505 \\
3059 \\
3120 \\
3202\end{array}$ & $\begin{array}{l}55 \\
132 \\
243 \\
327 \\
535 \\
858 \\
1060 \\
1250 \\
1369 \\
1486 \\
1512 \\
3066 \\
3131 \\
3786\end{array}$ & $\begin{array}{l}62 \\
140 \\
254 \\
377 \\
713 \\
917 \\
1079 \\
1253 \\
1372 \\
1497 \\
1627 \\
3071 \\
3138 \\
3950\end{array}$ & $\begin{array}{l}87 \\
167 \\
272 \\
407 \\
732 \\
939 \\
1121 \\
1268 \\
1410 \\
1498 \\
3045 \\
3090 \\
3142\end{array}$ \\
\hline DMTS••OH (TS3) & $\begin{array}{l}-1107 \\
113 \\
230 \\
343 \\
660 \\
856 \\
1059 \\
1133 \\
1272 \\
1396\end{array}$ & $\begin{array}{l}22 \\
125 \\
236 \\
378 \\
724 \\
876 \\
1081 \\
1143 \\
1327 \\
1413\end{array}$ & $\begin{array}{l}31 \\
139 \\
255 \\
421 \\
736 \\
918 \\
1086 \\
1230 \\
1337 \\
1417\end{array}$ & $\begin{array}{c}50 \\
160 \\
266 \\
504 \\
748 \\
933 \\
1102 \\
1250 \\
1347 \\
1443\end{array}$ & $\begin{array}{l}95 \\
205 \\
306 \\
509 \\
808 \\
1015 \\
1121 \\
1255 \\
1373 \\
1474\end{array}$ \\
\hline
\end{tabular}




\begin{tabular}{|c|c|c|c|c|c|}
\hline & $\begin{array}{l}1491 \\
3040 \\
3083 \\
3131\end{array}$ & $\begin{array}{l}1496 \\
3046 \\
3098 \\
3133\end{array}$ & $\begin{array}{l}1501 \\
3057 \\
3113 \\
3135\end{array}$ & $\begin{array}{l}1503 \\
3058 \\
3116 \\
3685\end{array}$ & $\begin{array}{l}1513 \\
3070 \\
3125\end{array}$ \\
\hline DMTS••OH (PC3) & $\begin{array}{l}18 \\
105 \\
196 \\
321 \\
493 \\
753 \\
996 \\
1122 \\
1269 \\
1418 \\
1497 \\
3046 \\
3094 \\
3136\end{array}$ & $\begin{array}{l}36 \\
124 \\
219 \\
352 \\
496 \\
858 \\
1061 \\
1164 \\
1329 \\
1459 \\
1505 \\
3056 \\
3113 \\
3190\end{array}$ & $\begin{array}{l}49 \\
141 \\
236 \\
368 \\
604 \\
877 \\
1068 \\
1205 \\
1374 \\
1476 \\
1512 \\
3061 \\
3122 \\
3704\end{array}$ & $\begin{array}{l}64 \\
169 \\
253 \\
394 \\
686 \\
912 \\
1082 \\
1241 \\
1387 \\
1482 \\
1647 \\
3066 \\
3125 \\
3958\end{array}$ & $\begin{array}{l}69 \\
190 \\
273 \\
430 \\
730 \\
918 \\
1121 \\
1250 \\
1417 \\
1491 \\
3006 \\
3079 \\
3128\end{array}$ \\
\hline $\mathrm{CH}_{3} \mathrm{C} \cdot \mathrm{HCH}_{2}-\mathrm{S}(\mathrm{O})-\mathrm{S}-\mathrm{C}_{3} \mathrm{H}_{7}$ & $\begin{array}{l}18 \\
136 \\
269 \\
486 \\
855 \\
1060 \\
1165 \\
1325 \\
1456 \\
1506 \\
3062 \\
3128\end{array}$ & $\begin{array}{l}46 \\
172 \\
323 \\
495 \\
869 \\
1065 \\
1194 \\
1372 \\
1473 \\
1510 \\
3071 \\
3130\end{array}$ & $\begin{array}{l}74 \\
195 \\
346 \\
612 \\
917 \\
1083 \\
1230 \\
1386 \\
1481 \\
3018 \\
3083 \\
3134\end{array}$ & $\begin{array}{l}86 \\
240 \\
389 \\
733 \\
920 \\
1121 \\
1251 \\
1418 \\
1488 \\
3048 \\
3093 \\
3135\end{array}$ & $\begin{array}{l}95 \\
252 \\
416 \\
748 \\
1000 \\
1160 \\
1268 \\
1421 \\
1493 \\
3059 \\
3116 \\
3208\end{array}$ \\
\hline DMTS••OH (TS4) & $\begin{array}{l}-883 \\
96 \\
239 \\
342 \\
614 \\
855 \\
1059 \\
1124 \\
1272 \\
1409 \\
1495 \\
3033\end{array}$ & $\begin{array}{l}27 \\
120 \\
249 \\
383 \\
708 \\
900 \\
1061 \\
1151 \\
1295 \\
1416 \\
1500 \\
3046\end{array}$ & $\begin{array}{l}44 \\
137 \\
254 \\
431 \\
732 \\
919 \\
1083 \\
1232 \\
1329 \\
1449 \\
1506 \\
3061\end{array}$ & $\begin{array}{l}74 \\
165 \\
260 \\
476 \\
756 \\
940 \\
1095 \\
1250 \\
1374 \\
1474 \\
1512 \\
3061\end{array}$ & $\begin{array}{c}89 \\
195 \\
312 \\
531 \\
810 \\
974 \\
1121 \\
1259 \\
1375 \\
1491 \\
1572 \\
3073\end{array}$ \\
\hline
\end{tabular}




\begin{tabular}{|c|c|c|c|c|c|}
\hline & $\begin{array}{l}3096 \\
3130\end{array}$ & $\begin{array}{l}3104 \\
3131\end{array}$ & $\begin{array}{l}3118 \\
3142\end{array}$ & $\begin{array}{l}3121 \\
3719\end{array}$ & 3125 \\
\hline \multirow[t]{14}{*}{ DMTS••OH (PC4) } & 19 & 30 & 53 & 68 & 79 \\
\hline & 83 & 101 & 123 & 133 & 139 \\
\hline & 166 & 207 & 239 & 250 & 258 \\
\hline & 345 & 355 & 378 & 394 & 420 \\
\hline & 495 & 529 & 573 & 621 & 730 \\
\hline & 748 & 856 & 868 & 906 & 918 \\
\hline & 1001 & 1059 & 1066 & 1084 & 1121 \\
\hline & 1122 & 1168 & 1196 & 1231 & 1251 \\
\hline & 1270 & 1328 & 1374 & 1387 & 1415 \\
\hline & 1416 & 1454 & 1473 & 1473 & 1495 \\
\hline & 1496 & 1503 & 1511 & 1644 & 3019 \\
\hline & 3058 & 3066 & 3067 & 3076 & 3087 \\
\hline & 3101 & 3122 & 3126 & 3134 & 3139 \\
\hline & 3143 & 3226 & 3736 & 3952 & \\
\hline \multirow[t]{14}{*}{ DMTS••OH (TS5) } & -828 & 17 & 31 & 45 & 63 \\
\hline & 93 & 103 & 124 & 146 & 185 \\
\hline & 190 & 237 & 252 & 260 & 300 \\
\hline & 334 & 344 & 379 & 423 & 505 \\
\hline & 715 & 726 & 737 & 751 & 790 \\
\hline & 857 & 877 & 917 & 920 & 1037 \\
\hline & 1060 & 1061 & 1083 & 1098 & 1120 \\
\hline & 1157 & 1169 & 1241 & 1251 & 1273 \\
\hline & 1277 & 1307 & 1327 & 1335 & 1372 \\
\hline & 1378 & 1416 & 1446 & 1459 & 1477 \\
\hline & 1479 & 1494 & 1496 & 1504 & 1513 \\
\hline & 3044 & 3044 & 3057 & 3070 & 3072 \\
\hline & 3077 & 3096 & 3110 & 3116 & 3125 \\
\hline & 3131 & 3146 & 3150 & 3795 & \\
\hline \multirow[t]{12}{*}{ DMTS••OH (PC5) } & 20 & 31 & 54 & 67 & 82 \\
\hline & 112 & 118 & 142 & 148 & 161 \\
\hline & 186 & 201 & 240 & 253 & 266 \\
\hline & 307 & 342 & 380 & 405 & 428 \\
\hline & 500 & 510 & 608 & 716 & 731 \\
\hline & 749 & 754 & 854 & 857 & 917 \\
\hline & 1003 & 1059 & 1065 & 1085 & 1092 \\
\hline & 1120 & 1121 & 1219 & 1249 & 1252 \\
\hline & 1270 & 1327 & 1329 & 1345 & 1373 \\
\hline & 1415 & 1445 & 1461 & 1473 & 1491 \\
\hline & 1496 & 1503 & 1510 & 1647 & 3057 \\
\hline & 3064 & 3067 & 3076 & 3085 & 3101 \\
\hline
\end{tabular}




\begin{tabular}{|c|c|c|c|c|c|}
\hline & $\begin{array}{l}3115 \\
3172\end{array}$ & $\begin{array}{l}3122 \\
3284\end{array}$ & $\begin{array}{l}3134 \\
3687\end{array}$ & $\begin{array}{l}3138 \\
3955\end{array}$ & 3156 \\
\hline \multirow[t]{12}{*}{$\mathrm{C} \cdot \mathrm{H}_{2} \mathrm{CH}_{2} \mathrm{CH}_{2}-\mathrm{S}(\mathrm{O})-\mathrm{S}-\mathrm{C}_{3} \mathrm{H}_{7}$} & 22 & 55 & 91 & 101 & 108 \\
\hline & 120 & 183 & 191 & 244 & 252 \\
\hline & 261 & 298 & 340 & 378 & 410 \\
\hline & 500 & 507 & 720 & 735 & 748 \\
\hline & 751 & 853 & 857 & 919 & 1002 \\
\hline & 1058 & 1060 & 1085 & 1091 & 1122 \\
\hline & 1167 & 1217 & 1235 & 1251 & 1270 \\
\hline & 1324 & 1328 & 1338 & 1374 & 1418 \\
\hline & 1445 & 1473 & 1474 & 1496 & 1501 \\
\hline & 1505 & 1512 & 3049 & 3050 & 3063 \\
\hline & 3070 & 3073 & 3098 & 3103 & 3119 \\
\hline & 3127 & 3132 & 3142 & 3174 & 3284 \\
\hline \multirow[t]{14}{*}{ DMTS••OH (TS6) } & -757 & 14 & 35 & 53 & 57 \\
\hline & 77 & 94 & 112 & 120 & 142 \\
\hline & 188 & 241 & 252 & 261 & 303 \\
\hline & 329 & 345 & 380 & 421 & 507 \\
\hline & 714 & 726 & 736 & 751 & 788 \\
\hline & 856 & 879 & 910 & 918 & 1035 \\
\hline & 1059 & 1072 & 1082 & 1100 & 1120 \\
\hline & 1154 & 1181 & 1251 & 1260 & 1271 \\
\hline & 1302 & 1328 & 1329 & 1337 & 1374 \\
\hline & 1384 & 1414 & 1446 & 1456 & 1473 \\
\hline & 1487 & 1493 & 1495 & 1504 & 1511 \\
\hline & 3057 & 3057 & 3066 & 3073 & 3076 \\
\hline & 3090 & 3100 & 3103 & 3121 & 3134 \\
\hline & 3138 & 3145 & 3162 & 3808 & \\
\hline \multirow{14}{*}{ DMTS••OH (PC6) } & 13 & 28 & 44 & 58 & 79 \\
\hline & 97 & 113 & 119 & 131 & 154 \\
\hline & 179 & 189 & 212 & 241 & 253 \\
\hline & 265 & 304 & 310 & 346 & 380 \\
\hline & 423 & 506 & 560 & 719 & 731 \\
\hline & 751 & 772 & 855 & 889 & 918 \\
\hline & 986 & 1059 & 1080 & 1095 & 1101 \\
\hline & 1121 & 1150 & 1168 & 1250 & 1258 \\
\hline & 1271 & 1306 & 1329 & 1374 & 1379 \\
\hline & 1414 & 1450 & 1467 & 1473 & 1473 \\
\hline & 1495 & 1504 & 1511 & 1611 & 3007 \\
\hline & 3057 & 3066 & 3073 & 3076 & 3080 \\
\hline & 3100 & 3121 & 3133 & 3137 & 3151 \\
\hline & 3160 & 3266 & 3831 & 3958 & \\
\hline
\end{tabular}




\begin{tabular}{|c|c|c|c|c|c|}
\hline DMTS••OH (RC3) & $\begin{array}{l}35 \\
99 \\
243 \\
348 \\
644 \\
858 \\
1060 \\
1123 \\
1329 \\
1418 \\
1504 \\
3060 \\
3103 \\
3141\end{array}$ & $\begin{array}{l}43 \\
118 \\
251 \\
384 \\
729 \\
868 \\
1073 \\
1242 \\
1331 \\
1452 \\
1506 \\
3064 \\
3109 \\
3142\end{array}$ & $\begin{array}{l}67 \\
133 \\
258 \\
423 \\
738 \\
920 \\
1082 \\
1250 \\
1374 \\
1474 \\
1510 \\
3065 \\
3120 \\
3144\end{array}$ & $\begin{array}{l}84 \\
182 \\
264 \\
496 \\
744 \\
923 \\
1111 \\
1259 \\
1377 \\
1494 \\
1512 \\
3075 \\
3132 \\
3529\end{array}$ & $\begin{array}{c}90 \\
209 \\
310 \\
515 \\
753 \\
1059 \\
1116 \\
1268 \\
1415 \\
1499 \\
3056 \\
3075 \\
3134\end{array}$ \\
\hline DMTS••OH (TS7) & $\begin{array}{l}-1084 \\
91 \\
236 \\
340 \\
686 \\
865 \\
1058 \\
1125 \\
1291 \\
1390 \\
1499 \\
3043 \\
3083 \\
3130\end{array}$ & $\begin{array}{c}18 \\
101 \\
252 \\
382 \\
732 \\
896 \\
1060 \\
1142 \\
1307 \\
1413 \\
1502 \\
3046 \\
3101 \\
3133\end{array}$ & $\begin{array}{c}54 \\
131 \\
262 \\
423 \\
749 \\
920 \\
1075 \\
1240 \\
1331 \\
1418 \\
1505 \\
3048 \\
3105 \\
3140\end{array}$ & $\begin{array}{c}62 \\
172 \\
265 \\
504 \\
751 \\
930 \\
1102 \\
1249 \\
1374 \\
1453 \\
1513 \\
3067 \\
3117 \\
3643\end{array}$ & $\begin{array}{r}88 \\
191 \\
312 \\
520 \\
814 \\
979 \\
1117 \\
1259 \\
1378 \\
1485 \\
1514 \\
3072 \\
3124\end{array}$ \\
\hline DMTS••OH (PC7) & $\begin{array}{l}11 \\
87 \\
184 \\
301 \\
450 \\
762 \\
1060 \\
1126 \\
1283 \\
1417 \\
1506 \\
3053 \\
3108 \\
3148\end{array}$ & $\begin{array}{l}37 \\
99 \\
236 \\
326 \\
478 \\
804 \\
1067 \\
1140 \\
1331 \\
1451 \\
1512 \\
3059 \\
3130 \\
3209\end{array}$ & $\begin{array}{l}58 \\
120 \\
251 \\
369 \\
557 \\
866 \\
1073 \\
1241 \\
1376 \\
1459 \\
1512 \\
3064 \\
3135 \\
3731\end{array}$ & $\begin{array}{l}77 \\
131 \\
254 \\
376 \\
730 \\
920 \\
1081 \\
1257 \\
1378 \\
1499 \\
1651 \\
3064 \\
3140 \\
3952\end{array}$ & $\begin{array}{l}80 \\
147 \\
262 \\
424 \\
749 \\
963 \\
1113 \\
1265 \\
1407 \\
1500 \\
3014 \\
3073 \\
3141\end{array}$ \\
\hline $\mathrm{H}_{7} \mathrm{C}_{3}-\mathrm{S}(\mathrm{O})-\mathrm{S}-\mathrm{C} \cdot \mathrm{HCH}_{2} \mathrm{CH}_{3}(\mathrm{P} 4)$ & 21 & 54 & 63 & 86 & 95 \\
\hline
\end{tabular}




\begin{tabular}{|c|c|c|c|c|c|}
\hline & $\begin{array}{l}108 \\
258 \\
427 \\
809 \\
1066 \\
1066 \\
1331 \\
1452 \\
1507 \\
3063 \\
3134\end{array}$ & $\begin{array}{l}156 \\
295 \\
482 \\
866 \\
1071 \\
1240 \\
1376 \\
1472 \\
1512 \\
3064 \\
3139\end{array}$ & $\begin{array}{c}239 \\
323 \\
728 \\
921 \\
1074 \\
1255 \\
1378 \\
1499 \\
3001 \\
3072 \\
3141\end{array}$ & $\begin{array}{c}243 \\
380 \\
749 \\
959 \\
1113 \\
1262 \\
1414 \\
1503 \\
3059 \\
3108 \\
3141\end{array}$ & $\begin{array}{l}252 \\
422 \\
760 \\
1060 \\
1140 \\
1283 \\
1417 \\
1505 \\
3062 \\
3130 \\
3200\end{array}$ \\
\hline DMTS••OH (TS8) & $\begin{array}{l}-641 \\
80 \\
202 \\
300 \\
645 \\
863 \\
1060 \\
1154 \\
1270 \\
1417 \\
1498 \\
3051 \\
3090 \\
3140\end{array}$ & $\begin{array}{l}21 \\
94 \\
229 \\
346 \\
728 \\
866 \\
1071 \\
1191 \\
1312 \\
1418 \\
1505 \\
3059 \\
3106 \\
3141\end{array}$ & $\begin{array}{l}22 \\
99 \\
249 \\
380 \\
748 \\
920 \\
1075 \\
1240 \\
1330 \\
1450 \\
1506 \\
3061 \\
3108 \\
3142\end{array}$ & $\begin{array}{l}37 \\
110 \\
252 \\
415 \\
755 \\
931 \\
1114 \\
1253 \\
1371 \\
1465 \\
1510 \\
3063 \\
3131 \\
3792\end{array}$ & $\begin{array}{l}56 \\
137 \\
259 \\
498 \\
779 \\
1055 \\
1119 \\
1256 \\
1375 \\
1484 \\
1512 \\
3073 \\
3134\end{array}$ \\
\hline DMTS••OH (PC8) & $\begin{array}{l}16 \\
85 \\
157 \\
256 \\
406 \\
756 \\
1060 \\
1142 \\
1278 \\
1417 \\
1505 \\
3059 \\
3106 \\
3145\end{array}$ & $\begin{array}{l}22 \\
92 \\
175 \\
300 \\
453 \\
813 \\
1062 \\
1163 \\
1331 \\
1450 \\
1508 \\
3061 \\
3130 \\
3182\end{array}$ & $\begin{array}{c}35 \\
95 \\
228 \\
308 \\
472 \\
866 \\
1072 \\
1241 \\
1376 \\
1470 \\
1512 \\
3062 \\
3134 \\
3847\end{array}$ & $\begin{array}{c}64 \\
129 \\
244 \\
355 \\
729 \\
921 \\
1077 \\
1255 \\
1379 \\
1499 \\
1616 \\
3071 \\
3139 \\
3960\end{array}$ & $\begin{array}{l}79 \\
153 \\
254 \\
381 \\
750 \\
956 \\
1112 \\
1258 \\
1414 \\
1501 \\
3000 \\
3079 \\
3140\end{array}$ \\
\hline DMTS••OH (TS9) & $\begin{array}{l}-796 \\
104 \\
210 \\
300\end{array}$ & $\begin{array}{l}28 \\
113 \\
248 \\
342\end{array}$ & $\begin{array}{l}38 \\
123 \\
250 \\
383\end{array}$ & $\begin{array}{l}63 \\
127 \\
260 \\
428\end{array}$ & $\begin{array}{l}90 \\
194 \\
287 \\
507\end{array}$ \\
\hline
\end{tabular}




\begin{tabular}{|c|c|c|c|c|c|}
\hline & $\begin{array}{l}667 \\
855 \\
1062 \\
1168 \\
1278 \\
1406 \\
1489 \\
3038 \\
3097 \\
3130\end{array}$ & $\begin{array}{l}737 \\
872 \\
1076 \\
1179 \\
1331 \\
1417 \\
1499 \\
3051 \\
3104 \\
3136\end{array}$ & $\begin{array}{l}751 \\
920 \\
1088 \\
1242 \\
1339 \\
1428 \\
1501 \\
3056 \\
3108 \\
3139\end{array}$ & $\begin{array}{c}754 \\
925 \\
1111 \\
1255 \\
1367 \\
1449 \\
1505 \\
3058 \\
3116 \\
3753\end{array}$ & $\begin{array}{l}772 \\
1054 \\
1120 \\
1257 \\
1377 \\
1464 \\
1513 \\
3067 \\
3124\end{array}$ \\
\hline DMTS••OH (PC9) & $\begin{array}{l}30 \\
115 \\
229 \\
349 \\
645 \\
855 \\
1063 \\
1122 \\
1324 \\
1417 \\
1503 \\
3042 \\
3092 \\
3122\end{array}$ & $\begin{array}{l}37 \\
121 \\
241 \\
385 \\
729 \\
866 \\
1076 \\
1242 \\
1329 \\
1451 \\
1503 \\
3051 \\
3095 \\
3128\end{array}$ & $\begin{array}{l}62 \\
136 \\
255 \\
422 \\
737 \\
919 \\
1078 \\
1249 \\
1371 \\
1471 \\
1511 \\
3053 \\
3109 \\
3136\end{array}$ & $\begin{array}{l}76 \\
174 \\
262 \\
497 \\
747 \\
922 \\
1116 \\
1260 \\
1374 \\
1495 \\
1512 \\
3062 \\
3119 \\
3528\end{array}$ & $\begin{array}{c}84 \\
204 \\
312 \\
519 \\
755 \\
1058 \\
1119 \\
1266 \\
1416 \\
1498 \\
3040 \\
3067 \\
3119\end{array}$ \\
\hline $\mathrm{H}_{7} \mathrm{C}_{3}-\mathrm{S}(\mathrm{O})-\mathrm{S}-\mathrm{CH}_{2} \mathrm{C} \cdot \mathrm{HCH}_{3}(\mathrm{P} 5)$ & $\begin{array}{l}40 \\
125 \\
300 \\
508 \\
851 \\
1059 \\
1166 \\
1328 \\
1450 \\
1506 \\
3060 \\
3122\end{array}$ & $\begin{array}{l}51 \\
167 \\
316 \\
550 \\
865 \\
1068 \\
1220 \\
1376 \\
1473 \\
1514 \\
3068 \\
3129\end{array}$ & $\begin{array}{l}80 \\
177 \\
345 \\
608 \\
896 \\
1080 \\
1239 \\
1391 \\
1481 \\
1516 \\
3079 \\
3137\end{array}$ & $\begin{array}{l}91 \\
256 \\
401 \\
727 \\
919 \\
1114 \\
1244 \\
1418 \\
1501 \\
3049 \\
3102 \\
3139\end{array}$ & $\begin{array}{l}98 \\
257 \\
419 \\
750 \\
991 \\
1158 \\
1250 \\
1418 \\
1501 \\
3058 \\
3117 \\
3204\end{array}$ \\
\hline DMTS••OH (TS10) & $\begin{array}{l}-709 \\
93 \\
232 \\
301 \\
677 \\
866 \\
1061\end{array}$ & $\begin{array}{l}17 \\
112 \\
251 \\
344 \\
721 \\
879 \\
1072\end{array}$ & $\begin{array}{l}31 \\
118 \\
254 \\
379 \\
730 \\
921 \\
1075\end{array}$ & $\begin{array}{l}55 \\
124 \\
257 \\
416 \\
746 \\
926 \\
1113\end{array}$ & $\begin{array}{c}85 \\
193 \\
299 \\
502 \\
751 \\
1043 \\
1122\end{array}$ \\
\hline
\end{tabular}




\begin{tabular}{|c|c|c|c|c|c|}
\hline & $\begin{array}{l}1161 \\
1271 \\
1411 \\
1495 \\
3052 \\
3097 \\
3140\end{array}$ & $\begin{array}{l}1185 \\
1331 \\
1417 \\
1499 \\
3059 \\
3108 \\
3142\end{array}$ & $\begin{array}{l}1241 \\
1337 \\
1452 \\
1506 \\
3064 \\
3119 \\
3145\end{array}$ & $\begin{array}{l}1244 \\
1371 \\
1460 \\
1512 \\
3065 \\
3125 \\
3768\end{array}$ & $\begin{array}{l}1256 \\
1376 \\
1492 \\
1527 \\
3073 \\
3131\end{array}$ \\
\hline DMTS••OH (PC10) & $\begin{array}{l}22 \\
93 \\
140 \\
291 \\
416 \\
750 \\
994 \\
1154 \\
1259 \\
1420 \\
1499 \\
3037 \\
3108 \\
3142\end{array}$ & $\begin{array}{l}26 \\
96 \\
165 \\
300 \\
477 \\
836 \\
1061 \\
1159 \\
1331 \\
1453 \\
1506 \\
3059 \\
3123 \\
3201\end{array}$ & $\begin{array}{l}48 \\
111 \\
184 \\
340 \\
507 \\
866 \\
1072 \\
1205 \\
1376 \\
1461 \\
1512 \\
3063 \\
3130 \\
3845\end{array}$ & $\begin{array}{c}70 \\
119 \\
252 \\
370 \\
704 \\
920 \\
1097 \\
1240 \\
1386 \\
1482 \\
1619 \\
3073 \\
3138 \\
3956\end{array}$ & \begin{tabular}{r}
\multicolumn{1}{c}{86} \\
127 \\
257 \\
393 \\
730 \\
926 \\
1116 \\
1255 \\
1417 \\
1490 \\
3002 \\
3084 \\
3139
\end{tabular} \\
\hline DMTS••OH (TS11) & $\begin{array}{l}-777 \\
89 \\
191 \\
335 \\
724 \\
865 \\
1059 \\
1168 \\
1267 \\
1377 \\
1488 \\
3048 \\
3084 \\
3130\end{array}$ & $\begin{array}{l}22 \\
95 \\
238 \\
346 \\
733 \\
867 \\
1061 \\
1194 \\
1319 \\
1418 \\
1500 \\
3063 \\
3104 \\
3141\end{array}$ & $\begin{array}{l}32 \\
110 \\
252 \\
378 \\
750 \\
919 \\
1073 \\
1239 \\
1326 \\
1450 \\
1501 \\
3064 \\
3105 \\
3152\end{array}$ & $\begin{array}{c}58 \\
121 \\
257 \\
418 \\
752 \\
945 \\
1085 \\
1250 \\
1329 \\
1453 \\
1505 \\
3072 \\
3123 \\
3806\end{array}$ & $\begin{array}{l}68 \\
149 \\
299 \\
504 \\
777 \\
1045 \\
1116 \\
1257 \\
1367 \\
1469 \\
1515 \\
3073 \\
3126\end{array}$ \\
\hline DMTS••OH (PC11) & $\begin{array}{l}12 \\
87 \\
187 \\
266 \\
419 \\
751 \\
1004 \\
1115\end{array}$ & $\begin{array}{l}25 \\
100 \\
193 \\
298 \\
503 \\
758 \\
1061 \\
1161\end{array}$ & $\begin{array}{l}41 \\
102 \\
246 \\
337 \\
597 \\
839 \\
1068 \\
1239\end{array}$ & $\begin{array}{l}52 \\
116 \\
248 \\
343 \\
719 \\
870 \\
1077 \\
1242\end{array}$ & $\begin{array}{l}79 \\
122 \\
260 \\
373 \\
729 \\
920 \\
1079 \\
1254\end{array}$ \\
\hline
\end{tabular}




\begin{tabular}{|c|c|c|c|c|c|}
\hline & $\begin{array}{l}1255 \\
1418 \\
1500 \\
3055 \\
3104 \\
3161\end{array}$ & $\begin{array}{l}1324 \\
1449 \\
1504 \\
3058 \\
3125 \\
3267\end{array}$ & $\begin{array}{l}1329 \\
1463 \\
1513 \\
3067 \\
3130 \\
3848\end{array}$ & $\begin{array}{l}1336 \\
1477 \\
1623 \\
3076 \\
3133 \\
3971\end{array}$ & $\begin{array}{l}1375 \\
1494 \\
3051 \\
3103 \\
3137\end{array}$ \\
\hline $\mathrm{H}_{7} \mathrm{C}_{3}-\mathrm{S}(\mathrm{O})-\mathrm{S}_{-}-\mathrm{CH}_{2} \mathrm{CH}_{2} \mathrm{CH}_{2}(\mathrm{P} 6)$ & $\begin{array}{l}23 \\
121 \\
259 \\
505 \\
758 \\
1059 \\
1170 \\
1319 \\
1452 \\
1506 \\
3070 \\
3130\end{array}$ & $\begin{array}{l}58 \\
162 \\
300 \\
517 \\
846 \\
1070 \\
1233 \\
1329 \\
1464 \\
1514 \\
3081 \\
3137\end{array}$ & $\begin{array}{l}91 \\
191 \\
345 \\
724 \\
865 \\
1072 \\
1240 \\
1333 \\
1477 \\
3048 \\
3102 \\
3138\end{array}$ & $\begin{array}{l}95 \\
254 \\
372 \\
736 \\
919 \\
1085 \\
1251 \\
1377 \\
1494 \\
3055 \\
3103 \\
3174\end{array}$ & $\begin{array}{l}111 \\
257 \\
418 \\
750 \\
1004 \\
1115 \\
1254 \\
1417 \\
1500 \\
3061 \\
3122 \\
3281\end{array}$ \\
\hline DMTS••OH (TS12) & $\begin{array}{l}-1219 \\
103 \\
251 \\
381 \\
667 \\
865 \\
1060 \\
1125 \\
1268 \\
1378 \\
1481 \\
3058 \\
3088 \\
3141\end{array}$ & $\begin{array}{c}32 \\
131 \\
258 \\
398 \\
728 \\
905 \\
1065 \\
1137 \\
1293 \\
1418 \\
1489 \\
3059 \\
3099 \\
3141\end{array}$ & $\begin{array}{l}45 \\
148 \\
278 \\
414 \\
747 \\
921 \\
1071 \\
1241 \\
1321 \\
1428 \\
1499 \\
3064 \\
3107 \\
3169\end{array}$ & $\begin{array}{l}67 \\
173 \\
308 \\
505 \\
752 \\
936 \\
1108 \\
1242 \\
1331 \\
1445 \\
1506 \\
3071 \\
3131 \\
3663\end{array}$ & $\begin{array}{c}84 \\
218 \\
342 \\
518 \\
775 \\
957 \\
1116 \\
1252 \\
1377 \\
1466 \\
1512 \\
3086 \\
3139\end{array}$ \\
\hline DMTS••OH (PC12) & $\begin{array}{l}26 \\
102 \\
201 \\
305 \\
482 \\
745 \\
988 \\
1117 \\
1260 \\
1418 \\
1499\end{array}$ & $\begin{array}{l}41 \\
107 \\
210 \\
343 \\
510 \\
752 \\
1060 \\
1121 \\
1307 \\
1451 \\
1506\end{array}$ & $\begin{array}{l}64 \\
129 \\
250 \\
352 \\
592 \\
866 \\
1072 \\
1188 \\
1331 \\
1461 \\
1512\end{array}$ & $\begin{array}{l}79 \\
157 \\
258 \\
386 \\
719 \\
883 \\
1087 \\
1242 \\
1376 \\
1468 \\
1633\end{array}$ & $\begin{array}{l}87 \\
169 \\
263 \\
421 \\
732 \\
921 \\
1094 \\
1256 \\
1377 \\
1473 \\
3009\end{array}$ \\
\hline
\end{tabular}




\begin{tabular}{|c|c|c|c|c|c|}
\hline & $\begin{array}{l}3059 \\
3118 \\
3166\end{array}$ & $\begin{array}{l}3065 \\
3131 \\
3278\end{array}$ & $\begin{array}{l}3074 \\
3134 \\
3745\end{array}$ & $\begin{array}{l}3079 \\
3141 \\
3935\end{array}$ & $\begin{array}{l}3108 \\
3142\end{array}$ \\
\hline \multirow[t]{14}{*}{ DMTS••OH (RC4) } & 22 & 27 & 51 & 64 & 81 \\
\hline & 96 & 98 & 121 & 174 & 206 \\
\hline & 240 & 251 & 253 & 261 & 303 \\
\hline & 358 & 386 & 417 & 505 & 556 \\
\hline & 631 & 723 & 733 & 746 & 752 \\
\hline & 853 & 869 & 917 & 922 & 1058 \\
\hline & 1060 & 1076 & 1083 & 1117 & 1119 \\
\hline & 1121 & 1242 & 1249 & 1263 & 1273 \\
\hline & 1328 & 1331 & 1374 & 1377 & 1415 \\
\hline & 1418 & 1455 & 1468 & 1494 & 1499 \\
\hline & 1504 & 1506 & 1510 & 1512 & 3058 \\
\hline & 3059 & 3062 & 3065 & 3070 & 3078 \\
\hline & 3099 & 3107 & 3124 & 3130 & 3134 \\
\hline & 3139 & 3139 & 3141 & 3619 & \\
\hline \multirow[t]{14}{*}{ DMTS••OH (TS13) } & -561 & 20 & 33 & 78 & 94 \\
\hline & 99 & 113 & 133 & 204 & 234 \\
\hline & 249 & 255 & 258 & 252 & 310 \\
\hline & 372 & 393 & 418 & 444 & 489 \\
\hline & 731 & 740 & 751 & 769 & 857 \\
\hline & 865 & 920 & 923 & 1049 & 1059 \\
\hline & 1063 & 1072 & 1075 & 1115 & 1121 \\
\hline & 1143 & 1242 & 1243 & 1254 & 1265 \\
\hline & 1328 & 1330 & 1373 & 1378 & 1414 \\
\hline & 1421 & 1454 & 1471 & 1496 & 1498 \\
\hline & 1506 & 1508 & 1512 & 1514 & 3045 \\
\hline & 3052 & 3052 & 3060 & 3062 & 3069 \\
\hline & 3096 & 3098 & 3119 & 3121 & 3127 \\
\hline & 3130 & 3132 & 3141 & 3670 & \\
\hline \multirow[t]{12}{*}{ DMTS••OH (PC13) } & 11 & 31 & 38 & 64 & 73 \\
\hline & 89 & 106 & 123 & 157 & 163 \\
\hline & 206 & 237 & 248 & 253 & 315 \\
\hline & 338 & 356 & 424 & 662 & 725 \\
\hline & 741 & 752 & 801 & 830 & 855 \\
\hline & 880 & 915 & 919 & 1057 & 1063 \\
\hline & 1066 & 1067 & 1111 & 1115 & 1239 \\
\hline & 1241 & 1252 & 1257 & 1303 & 1324 \\
\hline & 1326 & 1371 & 1378 & 1414 & 1417 \\
\hline & 1417 & 1451 & 1489 & 1497 & 1498 \\
\hline & 1504 & 1505 & 1511 & 1512 & 3053 \\
\hline & 3058 & 3059 & 3062 & 3064 & 3071 \\
\hline
\end{tabular}




\begin{tabular}{|c|c|c|c|c|c|}
\hline & $\begin{array}{l}3089 \\
3135\end{array}$ & $\begin{array}{l}3105 \\
3196\end{array}$ & $\begin{array}{l}3118 \\
3138\end{array}$ & $\begin{array}{l}3123 \\
3610\end{array}$ & 3131 \\
\hline \multirow[t]{6}{*}{$\mathrm{CH}_{3}-\mathrm{CH}_{2}-\mathrm{CH}_{2}-\mathrm{S}(=\mathrm{O})$} & 78 & 100 & 206 & 241 & 310 \\
\hline & 421 & 722 & 737 & 850 & 911 \\
\hline & 1054 & 1059 & 1103 & 1126 & 1241 \\
\hline & 1248 & 1324 & 1370 & 1416 & 1449 \\
\hline & 1497 & 1504 & 1510 & 3043 & 3048 \\
\hline & 3059 & 3097 & 3110 & 3123 & 3126 \\
\hline \multirow[t]{7}{*}{$\mathrm{CH}_{3}-\mathrm{CH}_{2}-\mathrm{CH}_{2}-\mathrm{S}-\mathrm{OH}$} & 79 & 103 & 223 & 235 & 294 \\
\hline & 398 & 407 & 721 & 749 & 806 \\
\hline & 856 & 922 & 1061 & 1080 & 1119 \\
\hline & 1177 & 1251 & 1268 & 1326 & 1370 \\
\hline & 1414 & 1449 & 1495 & 1503 & 1510 \\
\hline & 3042 & 3048 & 3059 & 3086 & 3109 \\
\hline & 3119 & 3123 & 3873 & & \\
\hline \multirow{14}{*}{ DMTS••OH (TS14) } & -499 & 38 & 43 & 83 & 97 \\
\hline & 102 & 114 & 142 & 189 & 206 \\
\hline & 249 & 254 & 259 & 274 & 285 \\
\hline & 323 & 327 & 389 & 416 & 474 \\
\hline & 728 & 739 & 741 & 753 & 862 \\
\hline & 868 & 912 & 917 & 923 & 1058 \\
\hline & 1058 & 1071 & 1075 & 1109 & 1113 \\
\hline & 1161 & 1237 & 1241 & 1252 & 1258 \\
\hline & 1324 & 1329 & 1372 & 1374 & 1416 \\
\hline & 1417 & 1460 & 1468 & 1492 & 1500 \\
\hline & 1505 & 1506 & 1511 & 1515 & 3047 \\
\hline & 3048 & 3051 & 3055 & 3064 & 3071 \\
\hline & 3095 & 3103 & 3115 & 3116 & 3124 \\
\hline & 3129 & 3134 & 3140 & 3859 & \\
\hline \multirow[t]{12}{*}{ DMTS••OH (TS15) } & -117 & 21 & 54 & 82 & 85 \\
\hline & 101 & 125 & 191 & 216 & 241 \\
\hline & 249 & 255 & 260 & 285 & 316 \\
\hline & 345 & 364 & 390 & 433 & 513 \\
\hline & 723 & 743 & 754 & 768 & 851 \\
\hline & 874 & 918 & 919 & 934 & 1059 \\
\hline & 1060 & 1082 & 1092 & 1118 & 1120 \\
\hline & 1129 & 1249 & 1251 & 1272 & 1273 \\
\hline & 1329 & 1331 & 1371 & 1378 & 1415 \\
\hline & 1416 & 1451 & 1461 & 1491 & 1502 \\
\hline & 1504 & 1505 & 1508 & 1514 & 3057 \\
\hline & 3058 & 3062 & 3078 & 3082 & 3097 \\
\hline
\end{tabular}




\begin{tabular}{|c|c|c|c|c|c|}
\hline & $\begin{array}{l}3099 \\
3139\end{array}$ & $\begin{array}{l}3119 \\
3141\end{array}$ & $\begin{array}{l}3126 \\
3169\end{array}$ & $\begin{array}{l}3115 \\
3861\end{array}$ & 3119 \\
\hline \multirow[t]{14}{*}{ DMTS••OH (RC4) } & 32 & 58 & 83 & 94 & 98 \\
\hline & 125 & 185 & 225 & 241 & 251 \\
\hline & 260 & 273 & 286 & 331 & 345 \\
\hline & 360 & 374 & 408 & 422 & 542 \\
\hline & 716 & 746 & 756 & 770 & 848 \\
\hline & 877 & 918 & 918 & 946 & 1059 \\
\hline & 1059 & 1066 & 1085 & 1104 & 1115 \\
\hline & 1123 & 1249 & 1255 & 1266 & 1273 \\
\hline & 1329 & 1332 & 1375 & 1377 & 1414 \\
\hline & 1416 & 1448 & 1453 & 1493 & 1501 \\
\hline & 1504 & 1506 & 1509 & 1513 & 3055 \\
\hline & 3058 & 3069 & 3075 & 3075 & 3096 \\
\hline & 3112 & 3113 & 3126 & 3135 & 3137 \\
\hline & 3139 & 3140 & 3180 & 3865 & \\
\hline \multirow[t]{14}{*}{ DMTS••OH (PC15) } & 23 & 32 & 52 & 70 & 96 \\
\hline & 97 & 111 & 131 & 164 & 197 \\
\hline & 237 & 248 & 253 & 261 & 309 \\
\hline & 347 & 367 & 417 & 540 & 701 \\
\hline & 719 & 742 & 748 & 778 & 826 \\
\hline & 864 & 918 & 921 & 1036 & 1059 \\
\hline & 1061 & 1073 & 1105 & 1106 & 1160 \\
\hline & 1198 & 1227 & 1241 & 1265 & 1277 \\
\hline & 1326 & 1328 & 1371 & 1376 & 1411 \\
\hline & 1414 & 1418 & 1463 & 1494 & 1500 \\
\hline & 1504 & 1506 & 1508 & 1515 & 3028 \\
\hline & 3051 & 3055 & 3072 & 3075 & 3085 \\
\hline & 3095 & 3110 & 3112 & 3129 & 3132 \\
\hline & 3135 & 3137 & 3142 & 3865 & \\
\hline \multirow[t]{8}{*}{$\mathrm{CH}_{3}-\mathrm{CH}_{2}-\mathrm{CH}_{2}-\mathrm{S}(=\mathrm{O}) \mathrm{OH}$} & 70 & 104 & 193 & 250 & 260 \\
\hline & 308 & 337 & 412 & 541 & 722 \\
\hline & 736 & 777 & 861 & 922 & 1059 \\
\hline & 1069 & 1106 & 1161 & 1218 & 1241 \\
\hline & 1275 & 1328 & 1377 & 1416 & 1454 \\
\hline & 1500 & 1505 & 1514 & 3056 & 3072 \\
\hline & 3090 & 3115 & 3129 & 3136 & 3146 \\
\hline & 3867 & & & & \\
\hline \multirow[t]{2}{*}{$\mathrm{CH}_{3}-\mathrm{CH}_{2}-\mathrm{CH}_{2}-\mathrm{S} \bullet$} & 109 & 239 & 243 & 373 & 374 \\
\hline & 750 & 767 & 918 & 967 & 1058 \\
\hline
\end{tabular}




\begin{tabular}{|c|c|c|c|c|c|}
\hline & $\begin{array}{l}1119 \\
1415 \\
3036 \\
3128\end{array}$ & $\begin{array}{l}1238 \\
1433 \\
3050 \\
3135\end{array}$ & $\begin{array}{l}1254 \\
1493 \\
3054\end{array}$ & $\begin{array}{l}1316 \\
1504 \\
3071\end{array}$ & $\begin{array}{l}1365 \\
1409 \\
3105\end{array}$ \\
\hline DMTS••Cl (TS16) & $\begin{array}{l}-224 \\
92 \\
230 \\
336 \\
583 \\
850 \\
1054 \\
1124 \\
1319 \\
1414 \\
1502 \\
3057 \\
3105 \\
3136\end{array}$ & $\begin{array}{l}35 \\
105 \\
238 \\
390 \\
690 \\
917 \\
1059 \\
1193 \\
1333 \\
1462 \\
1504 \\
3060 \\
3111 \\
3153\end{array}$ & $\begin{array}{c}62 \\
165 \\
254 \\
407 \\
723 \\
918 \\
1069 \\
1218 \\
1345 \\
1463 \\
1509 \\
3070 \\
3131 \\
3197\end{array}$ & $\begin{array}{c}69 \\
187 \\
284 \\
457 \\
743 \\
931 \\
1084 \\
1250 \\
1378 \\
1494 \\
1511 \\
3079 \\
3133 \\
3830\end{array}$ & $\begin{array}{l}79 \\
209 \\
309 \\
503 \\
774 \\
990 \\
1121 \\
1271 \\
1407 \\
1496 \\
3054 \\
3102 \\
3135\end{array}$ \\
\hline DMTS••Cl (PC16) & $\begin{array}{l}28 \\
78 \\
223 \\
322 \\
496 \\
798 \\
1043 \\
1123 \\
1318 \\
1413 \\
1500 \\
3055 \\
3112 \\
3142\end{array}$ & $\begin{array}{l}32 \\
82 \\
228 \\
362 \\
596 \\
846 \\
1059 \\
1197 \\
1330 \\
1456 \\
1504 \\
3056 \\
3128 \\
3163\end{array}$ & $\begin{array}{l}48 \\
102 \\
242 \\
369 \\
722 \\
895 \\
1083 \\
1227 \\
1340 \\
1470 \\
1508 \\
3056 \\
3131 \\
3165\end{array}$ & $\begin{array}{c}69 \\
119 \\
257 \\
396 \\
742 \\
912 \\
1106 \\
1253 \\
1371 \\
1486 \\
1508 \\
3078 \\
3133 \\
3777\end{array}$ & $\begin{array}{c}71 \\
213 \\
265 \\
469 \\
744 \\
920 \\
1121 \\
1274 \\
1399 \\
1492 \\
3054 \\
3093 \\
3135\end{array}$ \\
\hline
\end{tabular}


Table S6: Z-matrices for the optimized structures of all species and complexes at the MO6$2 X / 6-311++g(3 d f, 3 p d)$ level of theory.

\section{$\underline{\operatorname{DPTS}}\left(\mathrm{C}_{3} \underline{\mathrm{H}_{7}} \underline{\mathrm{S}} \underline{\mathrm{S}=\mathbf{O}) \mathbf{S C}_{3}} \underline{\underline{H}}_{7}\right)$}

Final structure in terms of initial Z-matrix:

$\mathrm{O}$

$\mathrm{S}, 1, \mathrm{~B} 1$

$\mathrm{C}, 2, \mathrm{~B} 2,1, \mathrm{~A} 1$

$\mathrm{H}, 3, \mathrm{~B} 3,2, \mathrm{~A} 2,1, \mathrm{D} 1,0$

$\mathrm{H}, 3, \mathrm{~B} 4,2, \mathrm{~A} 3,1, \mathrm{D} 2,0$

$\mathrm{C}, 3, \mathrm{~B} 5,2, \mathrm{~A} 4,1, \mathrm{D} 3,0$

$\mathrm{H}, 6, \mathrm{~B} 6,3, \mathrm{~A} 5,2, \mathrm{D} 4,0$

$\mathrm{H}, 6, \mathrm{~B} 7,3, \mathrm{~A} 6,2, \mathrm{D} 5,0$

$\mathrm{C}, 6, \mathrm{~B} 8,3, \mathrm{~A} 7,2, \mathrm{D} 6,0$

$\mathrm{H}, 9, \mathrm{~B} 9,6, \mathrm{~A} 8,3, \mathrm{D} 7,0$

H,9,B10,6,A9,3,D8,0

$\mathrm{H}, 9, \mathrm{~B} 11,6, \mathrm{~A} 10,3, \mathrm{D} 9,0$

$\mathrm{C}, 2, \mathrm{~B} 12,1, \mathrm{~A} 11,3, \mathrm{D} 10,0$

H,13,B13,2,A12,1,D11,0

H,13,B14,2,A13,1,D12,0

$\mathrm{C}, 13, \mathrm{~B} 15,2, \mathrm{~A} 14,1, \mathrm{D} 13,0$

H,16,B16,13,A15,2,D14,0

H,16,B17,13,A16,2,D15,0

C,16,B18,13,A17,2,D16,0

H,19,B19,16,A18,13,D17,0

H,19,B20,16,A19,13,D18,0

H,19,B21,16,A20,13,D19,0

S, 13,B22,2,A21,1,D20,0

Variables:

B1 $=1.47371257$

$\mathrm{B} 2=1.81212401$

$\mathrm{B} 3=1.09061803$

$\mathrm{B} 4=1.09039916$

$\mathrm{B} 5=1.52236508$

B6 $=1.09003372$

B7 $=1.09155099$

$\mathrm{B} 8=1.52479954$

B9 $=1.09002241$

$\mathrm{B} 10=1.08832119$

$\mathrm{B} 11=1.08976251$

$\mathrm{B} 12=2.92532483$

$\mathrm{B} 13=1.09095232$

B14 $=1.09020243$

$\mathrm{B} 15=1.52103035$ 


$$
\begin{aligned}
& \mathrm{B} 16=1.09083295 \\
& \mathrm{~B} 17=1.09036928 \\
& \mathrm{~B} 18=1.52511152 \\
& \mathrm{~B} 19=1.08848446 \\
& \mathrm{~B} 20=1.0903179 \\
& \mathrm{~B} 21=1.08991354 \\
& \mathrm{~B} 22=1.81600325 \\
& \mathrm{~A} 1=106.64323695 \\
& \mathrm{~A} 2=107.18847746 \\
& \text { A } 3=106.70253272 \\
& \text { A4 }=109.27029734 \\
& \text { A5 }=108.65336579 \\
& \text { A } 6=109.52046077 \\
& \mathrm{~A} 7=111.06206465 \\
& \mathrm{~A} 8=111.06360817 \\
& \text { A9 }=110.92235876 \\
& \mathrm{~A} 10=110.73772472 \\
& \text { A11 }=91.91764466 \\
& \mathrm{~A} 12=75.78888403 \\
& \mathrm{~A} 13=146.8394379 \\
& \text { A14 }=97.60632498 \\
& \mathrm{~A} 15=109.34645645 \\
& \mathrm{~A} 16=109.07316705 \\
& \text { A17 }=111.08686607 \\
& \text { A18 }=111.1195593 \\
& \text { A19 }=110.89331949 \\
& \text { A20 }=110.88179435 \\
& \text { A21 }=45.86217175 \\
& \text { D1 }=172.83331578 \\
& \text { D2 }=55.2993629 \\
& \text { D3 }=-65.37350754 \\
& \text { D4 }=55.82661943 \\
& \text { D5 }=-60.92629281 \\
& \text { D6 }=177.33627707 \\
& \mathrm{D} 7=59.87084221 \\
& \text { D8 }=-179.94499454 \\
& \text { D9 }=-60.01809446 \\
& \text { D10 }=134.58319342 \\
& \text { D1 } 1=12.87026874 \\
& \text { D12 }=-86.99780154 \\
& \text { D13 }=123.4321284 \\
& \text { D14 }=-14.75328853 \\
& \text { D15 }=101.6998791 \\
& \text { D16 }=-136.29272684 \\
& \text { D17 }=-179.8310834 \\
& \text { D18 }=-59.73180755
\end{aligned}
$$


D19 $=60.06647024$

D20 $=-119.4127228$

\section{$\underline{\text { DPTS••OH (TS1) }}$}

Final structure in terms of initial Z-matrix:

$\mathrm{O}$

$\mathrm{S}, 1, \mathrm{~B} 1$

C,2,B2,1,A1

$\mathrm{H}, 3, \mathrm{~B} 3,2, \mathrm{~A} 2,1, \mathrm{D} 1,0$

$\mathrm{H}, 3, \mathrm{~B} 4,2, \mathrm{~A} 3,1, \mathrm{D} 2,0$

$\mathrm{C}, 3, \mathrm{~B} 5,2, \mathrm{~A} 4,1, \mathrm{D} 3,0$

H,6,B6,3,A5,2,D4,0

H,6,B7,3,A6,2,D5,0

$\mathrm{C}, 6, \mathrm{~B} 8,3, \mathrm{~A} 7,2, \mathrm{D} 6,0$

$\mathrm{H}, 9, \mathrm{~B} 9,6, \mathrm{~A} 8,3, \mathrm{D} 7,0$

H,9,B10,6,A9,3,D8,0

H,9,B11,6,A10,3,D9,0

C,2,B12,1,A11,3,D10,0

$\mathrm{H}, 13, \mathrm{~B} 13,2, \mathrm{~A} 12,1, \mathrm{D} 11,0$

$\mathrm{H}, 13, \mathrm{~B} 14,2, \mathrm{~A} 13,1, \mathrm{D} 12,0$

C,13,B15,2,A14,1,D13,0

H,16,B16,13,A15,2,D14,0

H,16,B17,13,A16,2,D15,0

C,16,B18,13,A17,2,D16,0

H,19,B19,16,A18,13,D17,0

H,19,B20,16,A19,13,D18,0

H,19,B21,16,A20,13,D19,0

S, 13,B22,2,A21,1,D20,0

O,1,B23,2,A22,3,D21,0

H,2,B24, 1,A23,24,D22,0

Variables:

B1 $=1.48044377$

$\mathrm{B} 2=1.79701097$

$\mathrm{B} 3=1.08936142$

$\mathrm{B} 4=1.20003862$

$\mathrm{B} 5=1.51394405$

$\mathrm{B} 6=1.09053277$

$\mathrm{B} 7=1.09304749$

$\mathrm{B} 8=1.52534119$

$\mathrm{B} 9=1.09001922$

$\mathrm{B} 10=1.08809304$

$\mathrm{B} 11=1.08888321$

$\mathrm{B} 12=2.92490773$

$\mathrm{B} 13=1.09074099$

B14=1.09034974 


$$
\begin{aligned}
& \mathrm{B} 15=1.52081167 \\
& \mathrm{~B} 16=1.09080227 \\
& \text { B17 }=1.0903909 \\
& \text { B18 }=1.5249944 \\
& \mathrm{~B} 19=1.08833253 \\
& \mathrm{~B} 20=1.09017851 \\
& \text { B21 }=1.08996845 \\
& \mathrm{~B} 22=1.81762126 \\
& \mathrm{~B} 23=2.91773298 \\
& \mathrm{~B} 24=2.81238476 \\
& \mathrm{~A} 1=105.59128591 \\
& \mathrm{~A} 2=110.72267997 \\
& \mathrm{~A} 3=103.07000926 \\
& \mathrm{~A} 4=111.51919791 \\
& \text { A } 5=108.6561844 \\
& \text { A } 6=109.31968365 \\
& \mathrm{~A} 7=110.8728472 \\
& \mathrm{~A} 8=111.03311747 \\
& \text { A9 }=110.64565429 \\
& \mathrm{~A} 10=110.07895541 \\
& \text { A11 }=97.5953958 \\
& \text { A12 }=76.48630828 \\
& \text { A13 }=146.06294505 \\
& \text { A14 }=98.02266638 \\
& \text { A15 }=109.3047758 \\
& \text { A16 }=109.01398449 \\
& \text { A17 }=111.14878768 \\
& \text { A18 }=111.03710209 \\
& \text { A19 }=110.91268069 \\
& \text { A20 }=110.94400918 \\
& \text { A21 }=45.43269852 \\
& \text { A22 }=87.97732033 \\
& \text { A23 }=48.19446728 \\
& \text { D1 }=161.67964606 \\
& \text { D2 }=45.98250409 \\
& \text { D3 }=-69.15800513 \\
& \text { D4 }=49.44490743 \\
& \text { D5 }=-67.55620882 \\
& \text { D6 }=170.54863338 \\
& \text { D7 }=59.82215195 \\
& \text { D8 }=179.88438149 \\
& \text { D9 }=-60.19255349 \\
& \mathrm{D} 10=138.45962363 \\
& \text { D11 }=20.26662392 \\
& \text { D12 }=-80.25037032 \\
& \text { D13 }=130.90377706
\end{aligned}
$$




$$
\begin{aligned}
& \mathrm{D} 14=-15.08085087 \\
& \text { D15 }=101.33119318 \\
& \text { D16 }=-136.72622345 \\
& \text { D17 }=-179.84329407 \\
& \text { D18 }=-59.81121723 \\
& \text { D19 }=60.04741401 \\
& \text { D20 }=-112.3139159 \\
& \text { D21 }=-29.71821858 \\
& \text { D22 }=4.28709873
\end{aligned}
$$

\section{$\underline{\text { DPTS} \bullet O H ~(R C 1) ~}$}

Final structure in terms of initial Z-matrix:

$\mathrm{O}$

$\mathrm{S}, 1, \mathrm{~B} 1$

$\mathrm{C}, 2, \mathrm{~B} 2,1, \mathrm{~A} 1$

$\mathrm{H}, 3, \mathrm{~B} 3,2, \mathrm{~A} 2,1, \mathrm{D} 1,0$

$\mathrm{H}, 3, \mathrm{~B} 4,2, \mathrm{~A} 3,1, \mathrm{D} 2,0$

$\mathrm{C}, 3, \mathrm{~B} 5,2, \mathrm{~A} 4,1, \mathrm{D} 3,0$

$\mathrm{H}, 6, \mathrm{~B} 6,3, \mathrm{~A} 5,2, \mathrm{D} 4,0$

$\mathrm{H}, 6, \mathrm{~B} 7,3, \mathrm{~A} 6,2, \mathrm{D} 5,0$

C,6,B8,3,A7,2,D6,0

H,9,B9, 6,A8,3,D7,0

$\mathrm{H}, 9, \mathrm{~B} 10,6, \mathrm{~A} 9,3, \mathrm{D} 8,0$

H,9,B11,6,A10,3,D9,0

C,2,B12,1,A11,3,D10,0

H,13,B13,2,A12,1,D11,0

H,13,B14,2,A13,1,D12,0

C,13,B15,2,A14,1,D13,0

$\mathrm{H}, 16, \mathrm{~B} 16,13, \mathrm{~A} 15,2, \mathrm{D} 14,0$

H,16,B17,13,A16,2,D15,0

C,16,B18,13,A17,2,D16,0

$\mathrm{H}, 19, \mathrm{~B} 19,16, \mathrm{~A} 18,13, \mathrm{D} 17,0$

H,19,B20,16,A19,13,D18,0

H,19,B21,16,A20,13,D19,0

$\mathrm{S}, 13, \mathrm{~B} 22,2, \mathrm{~A} 21,1, \mathrm{D} 20,0$

O,3,B23,2,A22,1,D21,0

H,24,B24,3,A23, 2,D22,0

Variables:

$\mathrm{B} 1=1.48597572$

$\mathrm{B} 2=1.81229725$

$\mathrm{B} 3=1.09001095$

B4 $=1.09036459$

$\mathrm{B} 5=1.52360298$

B6 $=1.0908054$ 


$$
\begin{aligned}
& B 7=1.09145043 \\
& B 8=1.52441498 \\
& B 9=1.09018129 \\
& B 10=1.08813895 \\
& B 11=1.08945854 \\
& B 12=2.93219413 \\
& B 13=1.09074178 \\
& B 14=1.09029545 \\
& B 15=1.52094719 \\
& B 16=1.09074178 \\
& B 17=1.09038457 \\
& B 18=1.52502036 \\
& B 19=1.08834732 \\
& B 20=1.09017785 \\
& B 21=1.08994193 \\
& B 22=1.81718322 \\
& B 23=3.11586854 \\
& B 24=0.98329211 \\
& A 1=106.38529239 \\
& A 2=106.65930542 \\
& A 3=107.08953524 \\
& A 4=109.52322815 \\
& A 5=109.24065445 \\
& A 6=109.61147545 \\
& A 7=110.65881136 \\
& A 8=111.25554688 \\
& A 9=110.71019346 \\
& A 10=110.12865966 \\
& A 11=96.16015424 \\
& A 12=76.31566969 \\
& A 13=145.81675846 \\
& A 14=98.30307463 \\
& A 15=109.31191066 \\
& A 16=109.01553571 \\
& A 17=111.09348478 \\
& A 18=111.07595718 \\
& A 19=110.94838398 \\
& A 20=110.87604471 \\
& A 21=45.18228893 \\
& A 22=88.89578648 \\
& A 23=66.86028426 \\
& D 1=176.40867669 \\
& D 2=58.67620729 \\
& D 3=-61.80864895 \\
& D 4=50.89953582 \\
& D 5=-66.17448444
\end{aligned}
$$




$$
\begin{aligned}
& \text { D6 }=171.52470607 \\
& \text { D7 }=58.89499487 \\
& \text { D8 }=179.14605275 \\
& D 9=-61.33097583 \\
& \text { D10 }=137.13606117 \\
& \text { D1 }=19.37847427 \\
& \text { D12 }=-80.93059262 \\
& \text { D13 }=129.95226354 \\
& \text { D14 }=-14.68722843 \\
& \text { D15 }=101.74566927 \\
& \text { D16 }=-136.29733926 \\
& \text { D17 }=-179.93387077 \\
& \text { D18 }=-59.84328991 \\
& \text { D19 }=60.01893084 \\
& \text { D20 }=-113.62087087 \\
& \text { D21 }=11.63763908 \\
& \text { D22 }=-0.81234833
\end{aligned}
$$

\section{DPTS••OH (PC1)}

Final structure in terms of initial Z-matrix:

$\mathrm{O}$

$\mathrm{S}, 1, \mathrm{~B} 1$

$\mathrm{C}, 2, \mathrm{~B} 2,1, \mathrm{~A} 1$

$\mathrm{H}, 3, \mathrm{~B} 3,2, \mathrm{~A} 2,1, \mathrm{D} 1,0$

$\mathrm{H}, 3, \mathrm{~B} 4,2, \mathrm{~A} 3,1, \mathrm{D} 2,0$

$\mathrm{C}, 3, \mathrm{~B} 5,2, \mathrm{~A} 4,1, \mathrm{D} 3,0$

$\mathrm{H}, 6, \mathrm{~B} 6,3, \mathrm{~A} 5,2, \mathrm{D} 4,0$

H,6,B7,3,A6,2,D5,0

C,6,B8,3,A7,2,D6,0

H,9,B9, 6,A8,3,D7,0

H,9,B10,6,A9,3,D8,0

H,9,B11,6,A10,3,D9,0

C,2,B12,1,A11,3,D10,0

$\mathrm{H}, 13, \mathrm{~B} 13,2, \mathrm{~A} 12,1, \mathrm{D} 11,0$

$\mathrm{H}, 13, \mathrm{~B} 14,2, \mathrm{~A} 13,1, \mathrm{D} 12,0$

C,13,B15,2,A14,1,D13,0

$\mathrm{H}, 16, \mathrm{~B} 16,13, \mathrm{~A} 15,2, \mathrm{D} 14,0$

H,16,B17,13,A16,2,D15,0

$\mathrm{C}, 16, \mathrm{~B} 18,13, \mathrm{~A} 17,2, \mathrm{D} 16,0$

H,19,B19,16,A18,13,D17,0

H,19,B20,16,A19,13,D18,0

H,19,B21,16,A20,13,D19,0

S, 13,B22,2,A21,1,D20,0

O,3,B23,2,A22,1,D21,0

H,24,B24,3,A23,2,D22,0

Variables: 


$$
\begin{aligned}
& B 1=1.47865283 \\
& B 2=1.72796618 \\
& B 3=1.08244021 \\
& B 4=3.62593708 \\
& B 5=1.48332292 \\
& B 6=1.08845255 \\
& B 7=1.09573845 \\
& B 8=1.53183557 \\
& B 9=1.08978626 \\
& B 10=1.08850386 \\
& B 11=1.08703468 \\
& B 12=2.95363815 \\
& B 13=1.09088444 \\
& B 14=1.09053332 \\
& B 15=1.52068807 \\
& B 16=1.09099831 \\
& B 17=1.09047232 \\
& B 18=1.52494276 \\
& B 19=1.08836834 \\
& B 20=1.0902217 \\
& B 21=1.08995519 \\
& B 22=1.81913566 \\
& B 23=3.36874155 \\
& B 24=0.96783659 \\
& A 1=108.84033439 \\
& A 2=114.3912943 \\
& A 3=71.68020065 \\
& A 4=122.61913822 \\
& A 5=109.34988264 \\
& A 6=109.08601737 \\
& A 7=111.34194132 \\
& A 8=111.00198687 \\
& A 9=110.08533534 \\
& A 10=109.51762853 \\
& A 11=102.56643712 \\
& A 12=74.93024634 \\
& A 13=145.74503508 \\
& A 14=99.06269244 \\
& A 15=109.11458913 \\
& A 16=109.00439995 \\
& A 17=111.28125727 \\
& A 18=111.02707755 \\
& A 19=110.97076327 \\
& A 20=110.88427167 \\
& A 21=45.79420144 \\
& A 22=76.73973223 \\
& \text { a }
\end{aligned}
$$



A23 $=63.16696213$
D1 $=175.92490815$
$\mathrm{D} 2=59.22434005$
D3 $=-13.43352498$
D4 $=13.78933063$
D5 $=-103.13107328$
D6 $=136.01901667$
D7 $=57.03370325$
D8 $=176.82500145$
D9 $=-63.77411227$
$\mathrm{D} 10=143.08911996$
D1 $1=27.65681195$
D12 $=-70.53347755$
D13 $=137.80214674$
D14 $=-12.95803264$
D15 $=103.36729411$
D16 $=-134.58763636$
D17 $=-179.97274444$
D18 $=-59.90676586$
D19 $=59.97450439$
D20 $=-107.29163735$
D2 $1=44.32314777$
D22 $=-33.05514014$

\section{$\underline{\mathbf{C}}_{3} \underline{\mathrm{H}_{6}} \underline{\mathrm{S}(=\mathbf{O}) \mathbf{S C}_{3}} \underline{\mathrm{H}}_{7}$}

Final structure in terms of initial Z-matrix:

$\mathrm{O}$

$\mathrm{S}, 1, \mathrm{~B} 1$

$\mathrm{C}, 2, \mathrm{~B} 2,1, \mathrm{~A} 1$

H,3,B3, 2,A2, 1,D1,0

C,3,B4,2,A3, 1,D2,0

$\mathrm{H}, 5, \mathrm{~B} 5,3, \mathrm{~A} 4,2, \mathrm{D} 3,0$

$\mathrm{H}, 5, \mathrm{~B} 6,3, \mathrm{~A} 5,2, \mathrm{D} 4,0$

$\mathrm{C}, 5, \mathrm{~B} 7,3, \mathrm{~A} 6,2, \mathrm{D} 5,0$

$\mathrm{H}, 8, \mathrm{~B} 8,5, \mathrm{~A} 7,3, \mathrm{D} 6,0$

$\mathrm{H}, 8, \mathrm{~B} 9,5, \mathrm{~A} 8,3, \mathrm{D} 7,0$

$\mathrm{H}, 8, \mathrm{~B} 10,5, \mathrm{~A} 9,3, \mathrm{D} 8,0$

C,2,B11,1,A10,3,D9,0

$\mathrm{H}, 12, \mathrm{~B} 12,2, \mathrm{~A} 11,1, \mathrm{D} 10,0$

H, 12,B13,2,A12, 1,D11,0

C,12,B14,2,A13,1,D12,0

$\mathrm{H}, 15, \mathrm{~B} 15,12, \mathrm{~A} 14,2, \mathrm{D} 13,0$

$\mathrm{H}, 15, \mathrm{~B} 16,12, \mathrm{~A} 15,2, \mathrm{D} 14,0$

$\mathrm{C}, 15, \mathrm{~B} 17,12, \mathrm{~A} 16,2, \mathrm{D} 15,0$

H,18,B18,15,A17,12,D16,0 
H, 18,B19, 15,A18, 12,D17,0

H,18,B20,15,A19, 12,D18,0

S, 12,B21,2,A20,1,D19,0

Variables:

B1 $=1.47127585$

$\mathrm{B} 2=1.73140167$

$\mathrm{B} 3=1.08061941$

B4 $=1.48491795$

$\mathrm{B} 5=1.09105879$

B6 $=1.08902534$

$\mathrm{B} 7=1.53798587$

$\mathrm{B} 8=1.08909709$

B9 $=1.08880628$

$\mathrm{B} 10=1.08856288$

$\mathrm{B} 11=2.96174858$

$\mathrm{B} 12=1.09091862$

$\mathrm{B} 13=1.09066536$

$\mathrm{B} 14=1.52087653$

B15 $=1.0909099$

$\mathrm{B} 16=1.09050393$

$\mathrm{B} 17=1.5249297$

$\mathrm{B} 18=1.08851959$

$\mathrm{B} 19=1.09027982$

$\mathrm{B} 20=1.09007605$

$\mathrm{B} 21=1.81837755$

$\mathrm{A} 1=108.01774223$

$\mathrm{A} 2=115.70162302$

$\mathrm{A} 3=119.83499551$

A4 $=109.13850192$

A5 $=109.11351495$

A6 $=111.51012368$

A7 $=110.93971602$

$\mathrm{A} 8=110.06119854$

A9 $=109.85691953$

A10 $=100.33452084$

A11 $=75.25601743$

A12 $=146.10234511$

A13 $=98.68106196$

A14 $=109.13173366$

A15 $=108.98265139$

A16 $=111.33883607$

A17 $=111.10009989$

A18 $=110.93244438$

A19 $=110.9014158$

A20 $=45.85589978$

D1 $=-176.74056158$ 


$$
\begin{aligned}
& \mathrm{D} 2=8.18254199 \\
& \text { D3 }=-44.74743784 \\
& \text { D4 }=-162.71683431 \\
& \text { D5 }=74.9597681 \\
& \text { D6 }=59.54049226 \\
& \text { D7 }=179.85090984 \\
& \text { D8 }=-60.55631352 \\
& \text { D9 }=140.63141104 \\
& \text { D10 }=25.04982366 \\
& \text { D11 }=-73.59948118 \\
& \text { D12 }=135.31452832 \\
& \text { D13 }=-13.67416679 \\
& \text { D14 }=102.57790237 \\
& \text { D15 }=-135.32429836 \\
& \text { D16 }=179.73695881 \\
& \text { D17 }=-60.16686261 \\
& \text { D18 }=59.64532315 \\
& \text { D19 }=-109.24854277
\end{aligned}
$$

\section{$\underline{\text { DPTS••OH (TS2) }}$}

Final structure in terms of initial Z-matrix:

$\mathrm{O}$

$\mathrm{S}, 1, \mathrm{~B} 1$

$\mathrm{C}, 2, \mathrm{~B} 2,1, \mathrm{~A} 1$

$\mathrm{H}, 3, \mathrm{~B} 3,2, \mathrm{~A} 2,1, \mathrm{D} 1,0$

$\mathrm{H}, 3, \mathrm{~B} 4,2, \mathrm{~A} 3,1, \mathrm{D} 2,0$

C,3,B5,2,A4, 1,D3,0

$\mathrm{H}, 6, \mathrm{~B} 6,3, \mathrm{~A} 5,2, \mathrm{D} 4,0$

$\mathrm{H}, 6, \mathrm{~B} 7,3, \mathrm{~A} 6,2, \mathrm{D} 5,0$

$\mathrm{C}, 6, \mathrm{~B} 8,3, \mathrm{~A} 7,2, \mathrm{D} 6,0$

H,9,B9,6,A8,3,D7,0

H,9,B10,6,A9,3,D8,0

H,9,B11,6,A10,3,D9,0

$\mathrm{C}, 2, \mathrm{~B} 12,1, \mathrm{~A} 11,3, \mathrm{D} 10,0$

$\mathrm{H}, 13, \mathrm{~B} 13,2, \mathrm{~A} 12,1, \mathrm{D} 11,0$

H,13,B14,2,A13,1,D12,0

C,13,B15,2,A14,1,D13,0

$\mathrm{H}, 16, \mathrm{~B} 16,13, \mathrm{~A} 15,2, \mathrm{D} 14,0$

H,16,B17,13,A16,2,D15,0

$\mathrm{C}, 16, \mathrm{~B} 18,13, \mathrm{~A} 17,2, \mathrm{D} 16,0$

H,19,B19,16,A18,13,D17,0

H,19,B20,16,A19,13,D18,0

H,19,B21,16,A20,13,D19,0

S, 13,B22,2,A21,1,D20,0

$\mathrm{O}, 1, \mathrm{~B} 23,2, \mathrm{~A} 22,3, \mathrm{D} 21,0$ 
H,2,B24, 1,A23,24,D22,0

Variables:

B1 $=1.47974104$

$\mathrm{B} 2=1.81419489$

$\mathrm{B} 3=1.09233803$

B4 $=1.09070666$

B5 $=1.51326882$

$\mathrm{B} 6=1.18458983$

B7 $=1.09068727$

$\mathrm{B} 8=1.51443217$

B9 $=1.09194592$

B10 $=1.08768369$

B11 $=1.08943616$

$\mathrm{B} 12=2.92457449$

B13 $=1.09082462$

B14 $=1.09027257$

B15 $=1.52102724$

B16 $=1.09089077$

$\mathrm{B} 17=1.09039133$

$\mathrm{B} 18=1.52497762$

$\mathrm{B} 19=1.0883677$

$\mathrm{B} 20=1.09025552$

B21 $=1.08992886$

B22 $=1.81637988$

$\mathrm{B} 23=2.85525977$

B24 $=2.97686306$

$\mathrm{A} 1=107.21696919$

$\mathrm{A} 2=106.0537921$

$\mathrm{A} 3=107.57212217$

$\mathrm{A} 4=110.359794$

A5 $=107.60377943$

$\mathrm{A} 6=112.24571573$

$\mathrm{A} 7=112.79298182$

$\mathrm{A} 8=110.91582395$

A9 $=110.72667578$

A $10=110.35420129$

A11 $=93.59654839$

$\mathrm{A} 12=76.21565591$

A13 $=146.3271932$

A14 $=97.79902787$

A15 $=109.33608318$

A16 $=109.04630197$

A17 $=111.09259873$

A18 $=111.05178341$

$\mathrm{A} 19=110.90880809$

A20 $=110.92993754$ 


$$
\begin{aligned}
& \text { A2 } 1=45.53167952 \\
& \text { A22 }=108.38475966 \\
& \text { A23 }=35.69327755 \\
& \text { D1 }=173.76671983 \\
& \text { D2 }=56.441209 \\
& \text { D3 }=-64.93852176 \\
& \text { D4 }=65.45579602 \\
& \text { D5 }=-49.95024469 \\
& \text { D6 }=-178.76115752 \\
& \text { D7 }=62.76659149 \\
& \text { D8 }=-176.95267638 \\
& \text { D9 }=-57.23887785 \\
& \text { D10 }=135.81759226 \\
& \text { D1 } 1=15.72414879 \\
& \text { D12 }=-84.60581716 \\
& \text { D13 }=126.35842474 \\
& \text { D14 }=-14.78801051 \\
& \text { D15 }=101.65115937 \\
& \text { D16 }=-136.37379043 \\
& \text { D17 }=-179.73528457 \\
& \text { D18 }=-59.70545806 \\
& \text { D19 }=60.1451287 \\
& \text { D20 }=-116.74154627 \\
& \text { D21 }=21.15331784 \\
& \text { D22 }=1.89596241
\end{aligned}
$$

\section{$\underline{\text { DPTS } \bullet O H ~(R C 2) ~}$}

Final structure in terms of initial Z-matrix:

$\mathrm{O}$

$\mathrm{S}, 1, \mathrm{~B} 1$

$\mathrm{C}, 2, \mathrm{~B} 2,1, \mathrm{~A} 1$

$\mathrm{H}, 3, \mathrm{~B} 3,2, \mathrm{~A} 2,1, \mathrm{D} 1,0$

$\mathrm{H}, 3, \mathrm{~B} 4,2, \mathrm{~A} 3,1, \mathrm{D} 2,0$

C,3,B5,2,A4,1,D3,0

$\mathrm{H}, 6, \mathrm{~B} 6,3, \mathrm{~A} 5,2, \mathrm{D} 4,0$

H,6,B7,3,A6,2,D5,0

$\mathrm{C}, 6, \mathrm{~B} 8,3, \mathrm{~A} 7,2, \mathrm{D} 6,0$

$\mathrm{H}, 9, \mathrm{~B} 9,6, \mathrm{~A} 8,3, \mathrm{D} 7,0$

$\mathrm{H}, 9, \mathrm{~B} 10,6, \mathrm{~A} 9,3, \mathrm{D} 8,0$

H,9,B11,6,A10,3,D9,0

C,2,B12,1,A11,3,D10,0

$\mathrm{H}, 13, \mathrm{~B} 13,2, \mathrm{~A} 12,1, \mathrm{D} 11,0$

$\mathrm{H}, 13, \mathrm{~B} 14,2, \mathrm{~A} 13,1, \mathrm{D} 12,0$

C,13,B15,2,A14,1,D13,0

H,16,B16,13,A15,2,D14,0 


$$
\begin{aligned}
& \text { H,16,B17,13,A16,2,D15,0 } \\
& \text { C,16,B18,13,A17,2,D16,0 } \\
& \text { H,19,B19,16,A18,13,D17,0 } \\
& \text { H,19,B20,16,A19,13,D18,0 } \\
& \text { H,19,B21,16,A20,13,D19,0 } \\
& \text { S, 13,B22,2,A21,1,D20,0 } \\
& \mathrm{O}, 6, \mathrm{~B} 23,3, \mathrm{~A} 22,2, \mathrm{D} 21,0 \\
& \text { H,24,B24,6,A23,3,D22,0 } \\
& \text { Variables: } \\
& \mathrm{B} 1=1.48622795 \\
& \mathrm{~B} 2=1.81186154 \\
& \mathrm{~B} 3=1.09014842 \\
& \mathrm{~B} 4=1.09040415 \\
& \mathrm{~B} 5=1.52371039 \\
& \text { B6 }=1.09108284 \\
& \mathrm{~B} 7=1.09132681 \\
& \mathrm{~B} 8=1.52468871 \\
& \text { B9 }=1.09019456 \\
& \mathrm{~B} 10=1.08819245 \\
& \text { B11 }=1.08948361 \\
& \mathrm{~B} 12=2.92991998 \\
& \mathrm{~B} 13=1.09070359 \\
& \mathrm{~B} 14=1.09039788 \\
& \mathrm{~B} 15=1.52098044 \\
& \mathrm{~B} 16=1.09078217 \\
& \text { B17 }=1.09030808 \\
& \mathrm{~B} 18=1.52501571 \\
& \mathrm{~B} 19=1.08833357 \\
& \mathrm{~B} 20=1.09021323 \\
& \mathrm{~B} 21=1.08989527 \\
& \mathrm{~B} 22=1.81661104 \\
& \mathrm{~B} 23=3.07797433 \\
& \text { B24 }=0.98347633 \\
& \mathrm{~A} 1=106.3987119 \\
& \mathrm{~A} 2=106.75623937 \\
& \text { A3 }=106.97233052 \\
& \text { A4 }=109.59639245 \\
& \text { A } 5=109.3576673 \\
& \text { A6 }=109.56958777 \\
& \mathrm{~A} 7=110.53046628 \\
& \text { A } 8=111.17393208 \\
& \text { A } 9=110.79702398 \\
& \text { A } 10=110.0556761 \\
& \text { A11 }=94.76639545 \\
& \text { A12 }=77.06418906 \\
& \text { A13 }=146.39605246
\end{aligned}
$$



A14 $=97.32262233$
A $15=109.32376679$
$\mathrm{A} 16=109.08469612$
A17 $=111.07152418$
$\mathrm{A} 18=111.07312153$
A19 $=110.92236485$
$\mathrm{A} 20=110.91232536$
$\mathrm{A} 21=45.25224177$
A $22=77.2748775$
A23 $=78.64453959$
D1 $=174.81776314$
D2 $=57.09483889$
D3 $=-63.38511479$
D4 $=49.86625237$
D5 $=-67.29800457$
D6 $=170.51179507$
$\mathrm{D} 7=58.18555896$
D8 $=178.42254319$
D9 $=-61.96945791$
$\mathrm{D} 10=136.4561019$
D1 $1=16.34718033$
$\mathrm{D} 12=-85.21538264$
D13 $=127.08764408$
D14 $=-15.43906748$
D15 $=101.00982726$
D16 $=-136.99236828$
D17 $=179.98942103$
D18 $=-59.95316733$
D19 $=59.87319197$
D20 $=-115.32051712$
D21 $=83.26621948$
D22 $=-60.53521857$

\section{DPTS••OH (PC2)}

Final structure in terms of initial Z-matrix:

$\mathrm{O}$

$\mathrm{S}, 1, \mathrm{~B} 1$

$\mathrm{C}, 2, \mathrm{~B} 2,1, \mathrm{~A} 1$

$\mathrm{H}, 3, \mathrm{~B} 3,2, \mathrm{~A} 2,1, \mathrm{D} 1,0$

$\mathrm{H}, 3, \mathrm{~B} 4,2, \mathrm{~A} 3,1, \mathrm{D} 2,0$

C,3,B5,2,A4, 1,D3,0

$\mathrm{H}, 6, \mathrm{~B} 6,3, \mathrm{~A} 5,2, \mathrm{D} 4,0$

$\mathrm{H}, 6, \mathrm{~B} 7,3, \mathrm{~A} 6,2, \mathrm{D} 5,0$

$\mathrm{C}, 6, \mathrm{~B} 8,3, \mathrm{~A} 7,2, \mathrm{D} 6,0$

H,9,B9, 6,A8,3,D7,0 


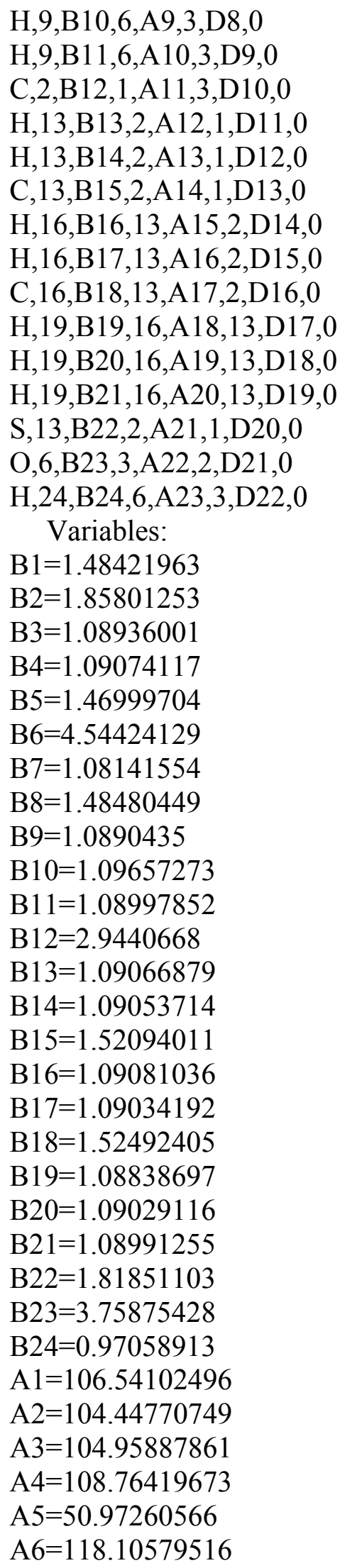



$\mathrm{A} 7=121.17291574$
A $8=111.2896475$
A $9=110.87758744$
$\mathrm{A} 10=111.04002842$
A11 $=97.64095184$
A12 $=76.97535927$
$\mathrm{A} 13=146.20252631$
A14 $=97.66661654$
$\mathrm{A} 15=109.22604383$
A16 $=109.03280227$
A $17=111.17598771$
A18 $=111.04911667$
A $19=110.95358356$
A $20=110.93809029$
A21 $=45.13059088$
$\mathrm{A} 22=58.56113545$
$\mathrm{A} 23=63.54018756$
$\mathrm{D} 1=177.33928217$
D2 $=59.82232305$
D3 $=-60.66174334$
D4 $=82.3226395$
D5 $=-85.02927584$
D6 $=83.36710697$
D7 $=156.12862463$
D8 $=-84.17386525$
D9 $=33.90239967$
D10 $=137.86467293$
D1 $1=20.84286911$
D12 $=-80.54539587$
D13 $=131.39868164$
D14 $=-15.49473879$
D15 $=100.8894646$
D16 $=-137.08662538$
$\mathrm{D} 17=179.73386325$
D18 $=-60.21377475$
D19 $=59.65909995$
D20 $=-111.54620933$
D21 $=83.23128365$
D22 $=-86.03058998$

\section{$\underline{\mathrm{CH}}_{3} \underline{\mathrm{CHCH}} \underline{2} \underline{\mathrm{S}} \underline{\mathrm{S}=\mathrm{O}) \mathrm{SC}_{3}} \underline{\mathrm{H}}_{7}$}

Final structure in terms of initial Z-matrix:

$\mathrm{O}$

$\mathrm{S}, 1, \mathrm{~B} 1$

C,2,B2,1,A1 
H,3,B3,2,A2,1,D1,0

$\mathrm{H}, 3, \mathrm{~B} 4,2, \mathrm{~A} 3,1, \mathrm{D} 2,0$

C,3,B5,2,A4,1,D3, 0

$\mathrm{H}, 6, \mathrm{~B} 6,3, \mathrm{~A} 5,2, \mathrm{D} 4,0$

$\mathrm{C}, 6, \mathrm{~B} 7,3, \mathrm{~A} 6,2, \mathrm{D} 5,0$

$\mathrm{H}, 8, \mathrm{~B} 8,6, \mathrm{~A} 7,3, \mathrm{D} 6,0$

$\mathrm{H}, 8, \mathrm{~B} 9,6, \mathrm{~A} 8,3, \mathrm{D} 7,0$

H,8,B10,6,A9,3,D8,0

$\mathrm{C}, 2, \mathrm{~B} 11,1, \mathrm{~A} 10,3, \mathrm{D} 9,0$

H,12,B12,2,A11,1,D10,0

$\mathrm{H}, 12, \mathrm{~B} 13,2, \mathrm{~A} 12,1, \mathrm{D} 11,0$

$\mathrm{C}, 12, \mathrm{~B} 14,2, \mathrm{~A} 13,1, \mathrm{D} 12,0$

H,15,B15,12,A14,2,D13,0

H,15,B16,12,A15,2,D14,0

C,15,B17,12,A16,2,D15,0

H,18,B18,15,A17,12,D16,0

H,18,B19, 15,A18,12,D17,0

H, 18,B20,15,A19, 12,D18,0

S, 12,B21,2,A20,1,D19,0

Variables:

$\mathrm{B} 1=1.47362036$

$\mathrm{B} 2=1.8549453$

B3 $=1.08987882$

B4 $=1.09047407$

B5 $=1.47016441$

B6 $=1.08095489$

$\mathrm{B} 7=1.48501503$

$\mathrm{B} 8=1.08813377$

B9 $=1.09597519$

$\mathrm{B} 10=1.0911982$

$\mathrm{B} 11=2.92776512$

B12 $=1.0908489$

$\mathrm{B} 13=1.09041286$

$\mathrm{B} 14=1.52112246$

$\mathrm{B} 15=1.09104393$

$\mathrm{B} 16=1.09034297$

$\mathrm{B} 17=1.52501399$

$\mathrm{B} 18=1.0885174$

$\mathrm{B} 19=1.09030534$

$\mathrm{B} 20=1.08991932$

$\mathrm{B} 21=1.81549529$

$\mathrm{A} 1=106.42558087$

A2 $=105.38981974$

$\mathrm{A} 3=104.40950642$

$\mathrm{A} 4=108.89950846$

A5 $=118.12850202$ 


$$
\begin{aligned}
& A 6=120.90225312 \\
& A 7=111.50945827 \\
& A 8=110.91470965 \\
& A 9=110.92366378 \\
& A 10=89.98947803 \\
& A 11=76.0874614 \\
& A 12=147.32132695 \\
& A 13=96.89425539 \\
& A 14=109.32608962 \\
& A 15=109.10313048 \\
& A 16=111.10878872 \\
& A 17=111.07773476 \\
& A 18=110.94400268 \\
& A 19=110.84298788 \\
& A 20=46.0159201 \\
& D 1=172.8180057 \\
& D 2=55.96459732 \\
& D 3=-64.66136227 \\
& D 4=-85.04591993 \\
& D 5=81.81328087 \\
& D 6=163.25833579 \\
& D 7=-76.10335333 \\
& D 8=41.71710397 \\
& D 9=133.42911067 \\
& D 10=9.95009599 \\
& D 11=-90.59791957 \\
& D 12=120.60829388 \\
& D 13=-14.68786038 \\
& D 14=101.75625355 \\
& D 15=-136.17676949 \\
& D 16=179.78032355 \\
& D 17=-60.09553786 \\
& D 18=59.7436613 \\
& D 19=-121.55558161 \\
& \text { D }
\end{aligned}
$$

\section{$\underline{\text { DPTS•・OH (TS3) }}$}

Final structure in terms of initial Z-matrix:

$\mathrm{O}$

$\mathrm{S}, 1, \mathrm{~B} 1$

$\mathrm{C}, 2, \mathrm{~B} 2,1, \mathrm{~A} 1$

H,3,B3, 2,A2, 1, D1,0

$\mathrm{H}, 3, \mathrm{~B} 4,2, \mathrm{~A} 3,1, \mathrm{D} 2,0$

$\mathrm{C}, 3, \mathrm{~B} 5,2, \mathrm{~A} 4,1, \mathrm{D} 3,0$

$\mathrm{H}, 6, \mathrm{~B} 6,3, \mathrm{~A} 5,2, \mathrm{D} 4,0$

H,6,B7,3,A6,2,D5,0 
C,6,B8,3,A7,2,D6,0

$\mathrm{H}, 9, \mathrm{~B} 9,6, \mathrm{~A} 8,3, \mathrm{D} 7,0$

H,9,B10,6,A9,3,D8,0

H,9,B11,6,A10,3,D9, 0

$\mathrm{C}, 2, \mathrm{~B} 12,1, \mathrm{~A} 11,3, \mathrm{D} 10,0$

H,13,B13,2,A12,1,D11,0

H,13,B14,2,A13,1,D12,0

C,13,B15,2,A14,1,D13,0

H,16,B16,13,A15,2,D14,0

H,16,B17,13,A16,2,D15,0

C,16,B18,13,A17,2,D16,0

H,19,B19,16,A 18, 13,D17,0

H, 19,B20,16,A19, 13,D18,0

H,19,B21,16,A20,13,D19,0

$\mathrm{S}, 13, \mathrm{~B} 22,2, \mathrm{~A} 21,1, \mathrm{D} 20,0$

$\mathrm{O}, 1, \mathrm{~B} 23,2, \mathrm{~A} 22,3, \mathrm{D} 21,0$

H, 2,B24, 1,A23, 24,D22,0

Variables:

$\mathrm{B} 1=1.47277346$

B2 $=1.8123987$

$\mathrm{B} 3=1.09068254$

B4 $=1.09007323$

$\mathrm{B} 5=1.5226263$

B6 $=1.08957509$

B7 $=1.09415438$

$\mathrm{B} 8=1.51424858$

B9 $=1.08884235$

$\mathrm{B} 10=1.08743028$

B11 $=1.1697888$

$\mathrm{B} 12=2.92830507$

$\mathrm{B} 13=1.09097965$

$\mathrm{B} 14=1.09025671$

$\mathrm{B} 15=1.5211654$

$\mathrm{B} 16=1.09084867$

B17 $=1.09046945$

$\mathrm{B} 18=1.52499315$

$\mathrm{B} 19=1.08849038$

B20 $=1.09031718$

$\mathrm{B} 21=1.08996213$

$\mathrm{B} 22=1.81592485$

$\mathrm{B} 23=5.10456466$

B24 $=5.42763981$

$\mathrm{A} 1=106.74180572$

$\mathrm{A} 2=106.86442224$

$\mathrm{A} 3=108.01110809$

$\mathrm{A} 4=108.85468263$ 


$$
\begin{aligned}
& \text { A5 }=108.85384902 \\
& \text { A6 }=109.89314874 \\
& \mathrm{~A} 7=111.24133519 \\
& \mathrm{~A} 8=113.14990824 \\
& \text { A } 9=113.5486431 \\
& \text { A10 }=107.49589936 \\
& \text { A11 }=94.99958503 \\
& \mathrm{~A} 12=75.89754095 \\
& \mathrm{~A} 13=146.51761714 \\
& \text { A14 }=97.89951432 \\
& \text { A15 }=109.26007967 \\
& \text { A16 }=109.05794971 \\
& \text { A17 }=111.16846328 \\
& \text { A } 18=111.12151262 \\
& \text { A19 }=110.90278401 \\
& \text { A20 }=110.89945426 \\
& \text { A21 }=45.66673854 \\
& \mathrm{~A} 22=76.62783932 \\
& \text { A23 }=95.9987334 \\
& \text { D1 }=176.41666732 \\
& \text { D2 }=58.12694331 \\
& \text { D3 }=-62.24117781 \\
& \text { D4 }=59.41968022 \\
& \text { D5 }=-57.57480396 \\
& \text { D6 }=-178.66894183 \\
& \mathrm{D} 7=58.43507987 \\
& \text { D8 }=-174.54590967 \\
& \text { D9 }=-58.86405398 \\
& \mathrm{D} 10=136.0810761 \\
& \text { D1 } 1=16.64338142 \\
& \text { D12 }=-83.16704096 \\
& \text { D13 }=127.1134749 \\
& \text { D14 }=-14.38373352 \\
& \text { D15 }=101.99355099 \\
& \text { D16 }=-135.99659889 \\
& \text { D17 }=-179.8872266 \\
& \text { D18 }=-59.82240162 \\
& \text { D19 }=59.95857846 \\
& \text { D20 }=-116.15525751 \\
& \text { D21 }=-10.12736221 \\
& \text { D22 }=1.33975838
\end{aligned}
$$

\section{$\underline{\text { DPTS••OH (RC3) }}$}

Final structure in terms of initial Z-matrix:

$\mathrm{O}$ 
$\mathrm{S}, 1, \mathrm{~B} 1$

C, 2,B2,1,A1

H,3,B3, 2,A2, 1,D1,0

H,3,B4, 2,A3,1,D2,0

C,3,B5,2,A4,1,D3,0

H,6,B6,3,A5,2,D4,0

H,6,B7,3,A6,2,D5,0

C,6,B8,3,A7,2,D6,0

H,9,B9,6,A8,3,D7,0

H,9,B10,6,A9,3,D8,0

H,9,B1 1,6,A10,3,D9,0

C,2,B12,1,A11,3,D10,0

H,13,B13,2,A12,1,D11,0

H,13,B14,2,A13,1,D12,0

C,13,B15,2,A14,1,D13,0

H,16,B16,13,A15,2,D14,0

H,16,B17,13,A 16,2,D15,0

C,16,B18,13,A17,2,D16,0

H,19,B19,16,A18,13,D17,0

H,19,B20,16,A19,13,D18,0

H,19,B21,16,A20,13,D19,0

S, 13,B22,2,A21,1,D20,0

O,3,B23,2,A22,1,D21,0

H,24,B24,3,A23, 2,D22,0

Variables:

$\mathrm{B} 1=1.48552401$

$\mathrm{B} 2=1.81083431$

$\mathrm{B} 3=1.09100954$

$\mathrm{B} 4=1.09124707$

$\mathrm{B} 5=1.52255458$

$\mathrm{B} 6=1.08999124$

B7 $=1.09145274$

$\mathrm{B} 8=1.52496024$

$\mathrm{B} 9=1.08992316$

$\mathrm{B} 10=1.08824485$

$\mathrm{B} 11=1.08955366$

$\mathrm{B} 12=2.9293575$

$\mathrm{B} 13=1.09076212$

B14 $=1.09019889$

B15 $=1.52094468$

$\mathrm{B} 16=1.09113956$

$\mathrm{B} 17=1.09035655$

$\mathrm{B} 18=1.52497275$

$\mathrm{B} 19=1.08838035$

$\mathrm{B} 20=1.09027017$

B21 $=1.08989244$ 


$$
\begin{aligned}
& \mathrm{B} 22=1.81512095 \\
& \mathrm{~B} 23=3.48665511 \\
& \text { B24 }=0.97941166 \\
& \mathrm{~A} 1=106.17640267 \\
& \text { A2 }=106.94632168 \\
& \mathrm{~A} 3=106.27675716 \\
& \mathrm{~A} 4=109.35237224 \\
& \text { A5 }=108.71517121 \\
& \mathrm{~A} 6=109.6622713 \\
& \mathrm{~A} 7=110.82435809 \\
& \text { A } 8=111.06641711 \\
& \text { A9 }=110.874331 \\
& \text { A } 10=110.70526392 \\
& \text { A11 }=88.41983622 \\
& \text { A12 }=76.99934092 \\
& \text { A13 }=146.79593742 \\
& \text { A14 }=96.56308378 \\
& \text { A15 }=109.39283324 \\
& \text { A16 }=109.1543873 \\
& \text { A17 }=111.02705581 \\
& \text { A18 }=111.06540542 \\
& \text { A19 }=110.9271828 \\
& \mathrm{~A} 20=110.83537072 \\
& \text { A21 }=45.60460483 \\
& \text { A22 }=69.69344977 \\
& \text { A23 }=62.43738857 \\
& \text { D1 }=172.65940731 \\
& \mathrm{D} 2=55.32736595 \\
& \text { D3 }=-65.63354292 \\
& \text { D4 }=55.91329198 \\
& \text { D5 }=-61.0372583 \\
& \text { D6 }=177.19534353 \\
& \text { D7 }=60.00684191 \\
& \text { D8 }=-179.84850772 \\
& \text { D9 }=-59.92397381 \\
& \text { D10 }=132.52869286 \\
& \text { D11 }=7.90063851 \\
& \text { D12 }=-93.67258119 \\
& \text { D13 }=118.92448302 \\
& \text { D14 }=-15.25212856 \\
& \text { D15 }=101.2675493 \\
& \text { D16 }=-136.67953619 \\
& \text { D17 }=-179.82689257 \\
& \text { D18 }=-59.717508 \\
& \text { D19 }=60.09314007 \\
& \text { D20 }=-122.62290262
\end{aligned}
$$


$\mathrm{D} 21=52.07359012$

D22 $=-45.94794941$

\section{$\underline{\text { DPTS・・OH (PC3) }}$}

Final structure in terms of initial Z-matrix:

$\mathrm{O}$

$\mathrm{S}, 1, \mathrm{~B} 1$

$\mathrm{C}, 2, \mathrm{~B} 2,1, \mathrm{~A} 1$

$\mathrm{H}, 3, \mathrm{~B} 3,2, \mathrm{~A} 2,1, \mathrm{D} 1,0$

$\mathrm{H}, 3, \mathrm{~B} 4,2, \mathrm{~A} 3,1, \mathrm{D} 2,0$

$\mathrm{C}, 3, \mathrm{~B} 5,2, \mathrm{~A} 4,1, \mathrm{D} 3,0$

$\mathrm{H}, 6, \mathrm{~B} 6,3, \mathrm{~A} 5,2, \mathrm{D} 4,0$

$\mathrm{H}, 6, \mathrm{~B} 7,3, \mathrm{~A} 6,2, \mathrm{D} 5,0$

$\mathrm{C}, 6, \mathrm{~B} 8,3, \mathrm{~A} 7,2, \mathrm{D} 6,0$

$\mathrm{H}, 9, \mathrm{~B} 9,6, \mathrm{~A} 8,3, \mathrm{D} 7,0$

$\mathrm{H}, 9, \mathrm{~B} 10,6, \mathrm{~A} 9,3, \mathrm{D} 8,0$

$\mathrm{H}, 9, \mathrm{~B} 11,6, \mathrm{~A} 10,3, \mathrm{D} 9,0$

$\mathrm{C}, 2, \mathrm{~B} 12,1, \mathrm{~A} 11,3, \mathrm{D} 10,0$

$\mathrm{H}, 13, \mathrm{~B} 13,2, \mathrm{~A} 12,1, \mathrm{D} 11,0$

$\mathrm{H}, 13, \mathrm{~B} 14,2, \mathrm{~A} 13,1, \mathrm{D} 12,0$

$\mathrm{C}, 13, \mathrm{~B} 15,2, \mathrm{~A} 14,1, \mathrm{D} 13,0$

$\mathrm{H}, 16, \mathrm{~B} 16,13, \mathrm{~A} 15,2, \mathrm{D} 14,0$

$\mathrm{H}, 16, \mathrm{~B} 17,13$,A16,2,D15,0

$\mathrm{C}, 16, \mathrm{~B} 18,13, \mathrm{~A} 17,2, \mathrm{D} 16,0$

H,19,B19,16,A18,13,D17,0

H,19,B20,16,A19,13,D18,0

H,19,B21,16,A20,13,D19,0

$\mathrm{S}, 13, \mathrm{~B} 22,2, \mathrm{~A} 21,1, \mathrm{D} 20,0$

$\mathrm{O}, 9, \mathrm{~B} 23,6, \mathrm{~A} 22,3, \mathrm{D} 21,0$

H,24,B24,9,A23,6,D22,0

Variables:

B1 $=1.48545713$

$\mathrm{B} 2=1.81469527$

$\mathrm{B} 3=1.08919579$

$\mathrm{B} 4=1.08942382$

B5 $=1.53666947$

B6 $=1.09077052$

B7 $=1.09083298$

$\mathrm{B} 8=1.4860742$

B9 $=1.07909861$

$\mathrm{B} 10=1.07884853$

B11 $=4.40586143$

$\mathrm{B} 12=2.92987542$

$\mathrm{B} 13=1.09078069$

B14=1.09032166 


$$
\begin{aligned}
& \mathrm{B} 15=1.52089139 \\
& \mathrm{~B} 16=1.09069171 \\
& \text { B17 }=1.09029311 \\
& \mathrm{~B} 18=1.52494144 \\
& \mathrm{~B} 19=1.08840371 \\
& \mathrm{~B} 20=1.09026536 \\
& \text { B21 }=1.08987465 \\
& \mathrm{~B} 22=1.8182668 \\
& \mathrm{~B} 23=3.5298693 \\
& \text { B24 }=0.97096225 \\
& \mathrm{~A} 1=106.43179887 \\
& \mathrm{~A} 2=106.93754704 \\
& \mathrm{~A} 3=107.21274528 \\
& \mathrm{~A} 4=109.13515214 \\
& \text { A5 }=107.90442887 \\
& \mathrm{~A} 6=109.38956211 \\
& \text { A7 }=110.11545716 \\
& \text { A8 }=118.95651468 \\
& \text { A9 }=121.27744191 \\
& \text { A } 10=68.4906215 \\
& \text { A11 }=96.16016291 \\
& \text { A12 }=76.57411026 \\
& \text { A13 }=146.26198968 \\
& \text { A14 }=97.72161944 \\
& \text { A15 }=109.24810758 \\
& \text { A16 }=109.01768396 \\
& \text { A17 }=111.17532547 \\
& \text { A18 }=111.04657154 \\
& \text { A19 }=110.99993292 \\
& \text { A20 }=110.86478035 \\
& \text { A21 }=45.2670002 \\
& \text { A22 }=63.10612856 \\
& \text { A23 }=100.01288748 \\
& \text { D1 }=176.02612269 \\
& \text { D2 }=57.35866108 \\
& \text { D3 }=-62.33613127 \\
& \mathrm{D} 4=51.44093647 \\
& \text { D5 }=-66.17800269 \\
& \text { D6 }=171.262599 \\
& \mathrm{D} 7=-75.82641008 \\
& \text { D8 }=94.04937064 \\
& \text { D9 }=-74.17451056 \\
& \text { D10 }=137.21872657 \\
& \text { D11 }=18.84754284 \\
& \text { D12 }=-81.96251416 \\
& \text { D13 }=129.47049992
\end{aligned}
$$




$$
\begin{aligned}
& \mathrm{D} 14=-13.65848899 \\
& \text { D15 }=102.71975716 \\
& \text { D16 }=-135.23817604 \\
& \text { D17 }=-179.53291951 \\
& \text { D18 }=-59.44474885 \\
& \text { D19 }=60.42308418 \\
& \text { D20 }=-113.60336099 \\
& \text { D21 }=-72.37325492 \\
& \text { D22 }=1.64210805
\end{aligned}
$$

\section{$\underline{\mathrm{CH}}_{2} \underline{\mathrm{CH}}_{2} \underline{\mathrm{CH}} 2 \underline{2} \underline{\mathrm{S}(=\mathrm{O}) \mathrm{SC}_{3}} \underline{\mathrm{H}}_{7}$}

Final structure in terms of initial Z-matrix:

$\mathrm{O}$

$\mathrm{S}, 1, \mathrm{~B} 1$

$\mathrm{C}, 2, \mathrm{~B} 2,1, \mathrm{~A} 1$

$\mathrm{H}, 3, \mathrm{~B} 3,2, \mathrm{~A} 2,1, \mathrm{D} 1,0$

$\mathrm{H}, 3, \mathrm{~B} 4,2, \mathrm{~A} 3,1, \mathrm{D} 2,0$

$\mathrm{C}, 3, \mathrm{~B} 5,2, \mathrm{~A} 4,1, \mathrm{D} 3,0$

$\mathrm{H}, 6, \mathrm{~B} 6,3, \mathrm{~A} 5,2, \mathrm{D} 4,0$

$\mathrm{H}, 6, \mathrm{~B} 7,3, \mathrm{~A} 6,2, \mathrm{D} 5,0$

$\mathrm{C}, 6, \mathrm{~B} 8,3, \mathrm{~A} 7,2, \mathrm{D} 6,0$

H,9,B9, 6,A8,3,D7,0

H,9,B10,6,A9,3,D8,0

C,2,B11,1,A10,3,D9,0

$\mathrm{H}, 12, \mathrm{~B} 12,2, \mathrm{~A} 11,1, \mathrm{D} 10,0$

$\mathrm{H}, 12, \mathrm{~B} 13,2, \mathrm{~A} 12,1, \mathrm{D} 11,0$

C,12,B14,2,A13,1,D12,0

$\mathrm{H}, 15, \mathrm{~B} 15,12, \mathrm{~A} 14,2, \mathrm{D} 13,0$

$\mathrm{H}, 15, \mathrm{~B} 16,12, \mathrm{~A} 15,2, \mathrm{D} 14,0$

$\mathrm{C}, 15, \mathrm{~B} 17,12, \mathrm{~A} 16,2, \mathrm{D} 15,0$

H,18,B18,15,A17,12,D16,0

H,18,B19,15,A18,12,D17,0

H,18,B20,15,A19,12,D18,0

S, 12,B21,2,A20,1,D19,0

Variables:

$\mathrm{B} 1=1.47337141$

$\mathrm{B} 2=1.81534426$

$\mathrm{B} 3=1.08953914$

$\mathrm{B} 4=1.08961819$

$\mathrm{B} 5=1.53618207$

$\mathrm{B} 6=1.09008679$

$\mathrm{B} 7=1.09191905$

$\mathrm{B} 8=1.4864671$

B9 $=1.07896838$

$\mathrm{B} 10=1.07913809$ 


$$
\begin{aligned}
& \mathrm{B} 11=2.92957715 \\
& \mathrm{~B} 12=1.090896 \\
& \mathrm{~B} 13=1.09032759 \\
& \mathrm{~B} 14=1.52112617 \\
& \text { B15 }=1.09088731 \\
& \text { B16 }=1.09043109 \\
& \mathrm{~B} 17=1.52501884 \\
& \mathrm{~B} 18=1.08844938 \\
& \mathrm{~B} 19=1.09028456 \\
& \mathrm{~B} 20=1.09000824 \\
& \mathrm{~B} 21=1.81642627 \\
& \mathrm{~A} 1=106.35666866 \\
& \mathrm{~A} 2=107.54617761 \\
& \mathrm{~A} 3=107.17591249 \\
& \text { A4 }=108.65753763 \\
& \text { A5 }=107.90128183 \\
& \text { A6 }=108.77362964 \\
& \text { A7 }=110.92973472 \\
& \mathrm{~A} 8=120.49272017 \\
& \text { A9 }=120.64713899 \\
& \text { A10 }=94.98615719 \\
& \text { A11 }=75.66417728 \\
& \text { A12 }=146.36791185 \\
& \text { A13 }=98.11539269 \\
& \text { A14 }=109.27877057 \\
& \text { A } 15=109.02587159 \\
& \text { A16 }=111.16542124 \\
& \text { A17 }=111.08605038 \\
& \text { A18 }=110.94899877 \\
& \text { A19 }=110.86308722 \\
& \text { A20 }=45.64745736 \\
& \text { D1 }=175.39546205 \\
& \text { D2 }=57.12461908 \\
& \text { D3 }=-63.14154656 \\
& \text { D4 }=56.34821637 \\
& \text { D5 }=-60.15871799 \\
& \text { D6 }=178.0487038 \\
& \text { D7 }=-85.99824111 \\
& \text { D8 }=84.16391791 \\
& \text { D9 }=135.93597735 \\
& \text { D10 }=17.10898541 \\
& \text { D11 }=-82.40335021 \\
& \text { D12 }=127.56776183 \\
& \text { D13 }=-13.88467353 \\
& \text { D14 }=102.5034397 \\
& \text { D15=-135.49514529 }
\end{aligned}
$$



D16 $=179.94595018$
$\mathrm{D} 17=-59.93533094$
$\mathrm{D} 18=59.90095822$
D19=-116.01734379

\section{DPTS••OH (TS4)}

Final structure in terms of initial Z-matrix:

$\mathrm{O}$

$\mathrm{S}, 1, \mathrm{~B} 1$

$\mathrm{C}, 2, \mathrm{~B} 2,1, \mathrm{~A} 1$

$\mathrm{H}, 3, \mathrm{~B} 3,2, \mathrm{~A} 2,1, \mathrm{D} 1,0$

H,3,B4,2,A3, 1,D2,0

C,3,B5,2,A4,1,D3,0

$\mathrm{H}, 6, \mathrm{~B} 6,3, \mathrm{~A} 5,2, \mathrm{D} 4,0$

$\mathrm{H}, 6, \mathrm{~B} 7,3, \mathrm{~A} 6,2, \mathrm{D} 5,0$

$\mathrm{C}, 6, \mathrm{~B} 8,3, \mathrm{~A} 7,2, \mathrm{D} 6,0$

$\mathrm{H}, 9, \mathrm{~B} 9,6, \mathrm{~A} 8,3, \mathrm{D} 7,0$

H,9,B10,6,A9,3,D8,0

H,9,B11,6,A10,3,D9,0

$\mathrm{C}, 2, \mathrm{~B} 12,1, \mathrm{~A} 11,3, \mathrm{D} 10,0$

H,13,B13,2,A12,1,D11,0

H,13,B14,2,A13,1,D12,0

C,13,B15,2,A14,1,D13,0

H,16,B16,13,A15,2,D14,0

H,16,B17,13,A16,2,D15,0

C,16,B18,13,A17,2,D16,0

H,19,B19,16,A18,13,D17,0

H,19,B20,16,A19, 13,D18,0

H,19,B21,16,A20,13,D19,0

S, 13,B22,2,A21,1,D20,0

$\mathrm{O}, 1, \mathrm{~B} 23,2, \mathrm{~A} 22,3, \mathrm{D} 21,0$

H,2,B24, 1,A23,24,D22,0

Variables:

$\mathrm{B} 1=1.48161212$

$\mathrm{B} 2=1.8099698$

$\mathrm{B} 3=1.09026929$

B4 $=1.0905285$

$\mathrm{B} 5=1.52270084$

B6 $=1.08982814$

$\mathrm{B} 7=1.09148159$

$\mathrm{B} 8=1.52490583$

$\mathrm{B} 9=1.08996973$

$\mathrm{B} 10=1.08813432$

$\mathrm{B} 11=1.08974641$

$\mathrm{B} 12=2.95378821$ 


$$
\begin{aligned}
& B 13=1.17586202 \\
& B 14=1.09076463 \\
& B 15=1.51511563 \\
& B 16=1.09068089 \\
& B 17=1.09213371 \\
& B 18=1.52475015 \\
& B 19=1.08827767 \\
& B 20=1.09023979 \\
& B 21=1.08891112 \\
& B 22=1.79410711 \\
& B 23=2.87728449 \\
& B 24=2.82081361 \\
& A 1=105.7735237 \\
& A 2=107.00874987 \\
& A 3=106.82832019 \\
& A 4=109.24305642 \\
& A 5=108.74884693 \\
& A 6=109.48527265 \\
& A 7=110.91229184 \\
& A 8=111.09566528 \\
& A 9=110.80907006 \\
& A 10=110.76518508 \\
& A 11=96.50142797 \\
& A 12=81.88527179 \\
& A 13=148.61079939 \\
& A 14=94.00313974 \\
& A 15=109.26804277 \\
& A 16=108.94263713 \\
& A 17=110.79306502 \\
& A 18=110.82677848 \\
& A 19=110.8575322 \\
& A 20=110.2123815 \\
& A 21=44.91734151 \\
& A 22=101.79781581 \\
& A 23=40.78720471 \\
& D 1=173.64467755 \\
& D 2=56.07292252 \\
& D 3=-64.65584331 \\
& D 4=56.17350303 \\
& D 5=-60.7015328 \\
& D 6=177.66674932 \\
& D 7=59.98767938 \\
& D 8=-179.86358788 \\
& D 9=-60.01938213 \\
& D 10=135.39960189 \\
& D 11=13.46018107
\end{aligned}
$$




$$
\begin{aligned}
& \mathrm{D} 12=-89.67226115 \\
& \mathrm{D} 13=121.19393715 \\
& \mathrm{D} 14=-18.56468225 \\
& \mathrm{D} 15=98.01463272 \\
& \mathrm{D} 16=-139.74831529 \\
& \mathrm{D} 17=179.28229551 \\
& \mathrm{D} 18=-60.79438922 \\
& \text { D19 }=59.02794636 \\
& \text { D20 }=-113.41143126 \\
& \text { D21 }=-152.51542399 \\
& \text { D22 }=1.38632752
\end{aligned}
$$

\section{$\underline{\text { DPTS } \bullet O H ~(R C 4) ~}$}

Final structure in terms of initial Z-matrix:

$\mathrm{O}$

$\mathrm{S}, 1, \mathrm{~B} 1$

$\mathrm{C}, 2, \mathrm{~B} 2,1, \mathrm{~A} 1$

$\mathrm{H}, 3, \mathrm{~B} 3,2, \mathrm{~A} 2,1, \mathrm{D} 1,0$

$\mathrm{H}, 3, \mathrm{~B} 4,2, \mathrm{~A} 3,1, \mathrm{D} 2,0$

$\mathrm{C}, 3, \mathrm{~B} 5,2, \mathrm{~A} 4,1, \mathrm{D} 3,0$

$\mathrm{H}, 6, \mathrm{~B} 6,3, \mathrm{~A} 5,2, \mathrm{D} 4,0$

H,6,B7,3,A6,2,D5,0

$\mathrm{C}, 6, \mathrm{~B} 8,3, \mathrm{~A} 7,2, \mathrm{D} 6,0$

H,9,B9,6,A8,3,D7,0

H,9,B10,6,A9,3,D8,0

H,9,B11,6,A10,3,D9,0

C,2,B12,1,A11,3,D10,0

$\mathrm{H}, 13, \mathrm{~B} 13,2, \mathrm{~A} 12,1, \mathrm{D} 11,0$

$\mathrm{H}, 13, \mathrm{~B} 14,2, \mathrm{~A} 13,1, \mathrm{D} 12,0$

C,13,B15,2,A14,1,D13,0

H,16,B16,13,A15,2,D14,0

H,16,B17,13,A16,2,D15,0

$\mathrm{C}, 16, \mathrm{~B} 18,13, \mathrm{~A} 17,2, \mathrm{D} 16,0$

H,19,B19,16,A18,13,D17,0

H,19,B20,16,A19,13,D18,0

H,19,B21,16,A20,13,D19,0

S, 13,B22,2,A21,1,D20,0

$\mathrm{O}, 1, \mathrm{~B} 23,2, \mathrm{~A} 22,3, \mathrm{D} 21,0$

H,24,B24,1,A23,2,D22,0

Variables:

$\mathrm{B} 1=1.4884529$

B2 $=1.80774181$

$\mathrm{B} 3=1.09052927$

B4 $=1.09030264$

B5 $=1.52268015$ 

B6 $=1.0898359$
B7 $=1.09139608$
$\mathrm{B} 8=1.52481506$
B9 $=1.08997142$
$\mathrm{B} 10=1.08814433$
$\mathrm{B} 11=1.08972446$
$\mathrm{B} 12=2.93735631$
$\mathrm{B} 13=1.09151802$
B14 $=1.09082674$
$\mathrm{B} 15=1.52060277$
$\mathrm{B} 16=1.09033262$
$\mathrm{B} 17=1.09067909$
B18 $=1.52474401$
B19 $=1.08849664$
$\mathrm{B} 20=1.09065544$
B21 $=1.08931063$
$\mathrm{B} 22=1.81839188$
$\mathrm{B} 23=2.77366824$
B24 $=0.98361045$
$\mathrm{A} 1=105.61805797$
$\mathrm{A} 2=106.92642635$
A $3=106.74863888$
$\mathrm{A} 4=109.28707353$
A $5=108.6743504$
$\mathrm{A} 6=109.50685031$
A $7=110.93803625$
A8 $=111.07571858$
A9 $=110.81582454$
$\mathrm{A} 10=110.83543818$
A11 $=92.31761984$
$\mathrm{A} 12=80.53488355$
$\mathrm{A} 13=147.28186928$
A14 $=94.548421$
A $15=109.38457465$
$\mathrm{A} 16=109.08545427$
A17 $=110.64756389$
A18 $=110.83899506$
A19 $=111.02205619$
A20 $=110.36655395$
A21 $=45.06866018$
A22 $=99.99726838$
A23 $=13.81465026$
$\mathrm{D} 1=173.23763241$
D2 $=55.77404221$
D3 $=-65.09951988$
D4 $=57.20622586$ 


$$
\begin{aligned}
& \mathrm{D} 5=-59.60433336 \\
& \text { D6 }=178.70557767 \\
& \text { D7 }=60.38839559 \\
& \text { D8 }=-179.52149785 \\
& \text { D9 }=-59.63079933 \\
& \text { D10 }=135.20019857 \\
& \text { D1 } 1=8.84746017 \\
& \text { D12 }=-97.79589859 \\
& \text { D13 }=120.06292179 \\
& \text { D14 }=-18.65334607 \\
& \text { D15 }=98.55272263 \\
& \text { D16 }=-139.24621713 \\
& \text { D17 }=-179.84028077 \\
& \text { D18 }=-59.8010111 \\
& \text { D19 }=60.5169459 \\
& \text { D20 }=-118.43486421 \\
& \text { D21 }=175.31989243 \\
& \text { D22 }=-153.15143228
\end{aligned}
$$

\section{$\underline{\text { DPTS } \bullet O H ~(P C 4) ~}$}

Final structure in terms of initial Z-matrix:

$\mathrm{O}$

$\mathrm{S}, 1, \mathrm{~B} 1$

$\mathrm{C}, 2, \mathrm{~B} 2,1, \mathrm{~A} 1$

$\mathrm{H}, 3, \mathrm{~B} 3,2, \mathrm{~A} 2,1, \mathrm{D} 1,0$

$\mathrm{H}, 3, \mathrm{~B} 4,2, \mathrm{~A} 3,1, \mathrm{D} 2,0$

$\mathrm{C}, 3, \mathrm{~B} 5,2, \mathrm{~A} 4,1, \mathrm{D} 3,0$

$\mathrm{H}, 6, \mathrm{~B} 6,3, \mathrm{~A} 5,2, \mathrm{D} 4,0$

$\mathrm{H}, 6, \mathrm{~B} 7,3, \mathrm{~A} 6,2, \mathrm{D} 5,0$

$\mathrm{C}, 6, \mathrm{~B} 8,3, \mathrm{~A} 7,2, \mathrm{D} 6,0$

$\mathrm{H}, 9, \mathrm{~B} 9,6, \mathrm{~A} 8,3, \mathrm{D} 7,0$

H,9,B10,6,A9,3,D8, 0

$\mathrm{H}, 9, \mathrm{~B} 11,6, \mathrm{~A} 10,3, \mathrm{D} 9,0$

$\mathrm{C}, 2, \mathrm{~B} 12,1, \mathrm{~A} 11,3, \mathrm{D} 10,0$

$\mathrm{H}, 1, \mathrm{~B} 13,2, \mathrm{~A} 12,3, \mathrm{D} 11,0$

$\mathrm{H}, 13, \mathrm{~B} 14,2, \mathrm{~A} 13,1, \mathrm{D} 12,0$

$\mathrm{C}, 13, \mathrm{~B} 15,2, \mathrm{~A} 14,1, \mathrm{D} 13,0$

$\mathrm{H}, 16, \mathrm{~B} 16,13, \mathrm{~A} 15,2, \mathrm{D} 14,0$

$\mathrm{H}, 16, \mathrm{~B} 17,13$,A16,2,D15,0

C,16,B18,13,A17,2,D16,0

H,19,B19,16,A18,13,D17,0

$\mathrm{H}, 19, \mathrm{~B} 20,16, \mathrm{~A} 19,13$,D18, 0

H,19,B21,16,A20,13,D19,0

$\mathrm{S}, 13, \mathrm{~B} 22,2, \mathrm{~A} 21,1, \mathrm{D} 20,0$

$\mathrm{O}, 1, \mathrm{~B} 23,2, \mathrm{~A} 22,3, \mathrm{D} 21,0$ 
H,24,B24,1,A23,2,D22,0

Variables:

B1 $=1.48406513$

$\mathrm{B} 2=1.80852579$

$\mathrm{B} 3=1.09098428$

B4 $=1.09052506$

$\mathrm{B} 5=1.52284483$

$\mathrm{B} 6=1.08985342$

B7 $=1.0913973$

$\mathrm{B} 8=1.52486429$

B9 $=1.08986227$

$\mathrm{B} 10=1.08828877$

B11 $=1.08972873$

$\mathrm{B} 12=2.97900741$

$\mathrm{B} 13=3.32052443$

B14 $=1.08075334$

$\mathrm{B} 15=1.48801543$

B16 $=1.09663641$

B17 $=1.09402447$

$\mathrm{B} 18=1.52378551$

$\mathrm{B} 19=1.08835044$

$\mathrm{B} 20=1.08977044$

$\mathrm{B} 21=1.08843976$

B22 $=1.709117$

$\mathrm{B} 23=2.86067826$

B24 $=0.96945112$

$\mathrm{A} 1=105.88318732$

$\mathrm{A} 2=106.79260035$

$\mathrm{A} 3=106.94029739$

$\mathrm{A} 4=109.53871565$

A5 $=108.67483722$

$\mathrm{A} 6=109.49367437$

A7 $=111.0776964$

A $8=111.08780422$

A9 $=110.7901754$

A10 $=110.83151634$

A11 $=94.06622394$

$\mathrm{A} 12=125.58301365$

$\mathrm{A} 13=130.53006077$

A14 $=90.64327713$

A15 $=109.14449463$

A16 $=109.24410823$

A17 $=111.88409895$

A18 $=110.85082215$

A19 $=110.89295717$

A20 $=109.48040699$ 

$\mathrm{A} 21=45.52580331$
$\mathrm{A} 22=111.35863196$
A23 $=6.49087315$
D1 $=173.04328516$
D2 $=55.72277054$
D3 $=-65.27195282$
D4 $=56.87156119$
D5 $=-59.88354708$
D6 $=178.36183897$
D7 $=60.17486829$
D8 $=-179.72066941$
D9 $=-59.84794731$
D10 $=130.84914669$
D11 $=-164.33282348$
D12 $=-29.21160143$
$\mathrm{D} 13=102.89382258$
D14 $=-31.79889809$
D15 $=83.87235162$
D16 $=-152.2762249$
D17 $=-179.80328353$
D18 $=-59.56198976$
D19 $=60.57368366$
D20 $=-117.600347$
D21 $=-156.80169704$
D22 $=169.54596726$

\section{$\underline{\mathrm{C}}_{3} \underline{\mathrm{H}_{7}} \underline{\mathrm{S}} \underline{\mathrm{S}=\mathbf{O}) \mathrm{SCHCH}_{2}} \underline{\mathrm{CH}}_{3}$}

Final structure in terms of initial Z-matrix:

$\mathrm{O}$

$\mathrm{S}, 1, \mathrm{~B} 1$

$\mathrm{C}, 2, \mathrm{~B} 2,1, \mathrm{~A} 1$

$\mathrm{H}, 3, \mathrm{~B} 3,2, \mathrm{~A} 2,1, \mathrm{D} 1,0$

$\mathrm{H}, 3, \mathrm{~B} 4,2, \mathrm{~A} 3,1, \mathrm{D} 2,0$

$\mathrm{C}, 3, \mathrm{~B} 5,2, \mathrm{~A} 4,1, \mathrm{D} 3,0$

$\mathrm{H}, 6, \mathrm{~B} 6,3, \mathrm{~A} 5,2, \mathrm{D} 4,0$

H,6,B7,3,A6,2,D5,0

$\mathrm{C}, 6, \mathrm{~B} 8,3, \mathrm{~A} 7,2, \mathrm{D} 6,0$

$\mathrm{H}, 9, \mathrm{~B} 9,6, \mathrm{~A} 8,3, \mathrm{D} 7,0$

$\mathrm{H}, 9, \mathrm{~B} 10,6, \mathrm{~A} 9,3, \mathrm{D} 8,0$

H,9,B11,6,A10,3,D9,0

C,2,B12,1,A11,3,D10,0

$\mathrm{H}, 13, \mathrm{~B} 13,2, \mathrm{~A} 12,1, \mathrm{D} 11,0$

C,13,B14,2,A13,1,D12,0

$\mathrm{H}, 15, \mathrm{~B} 15,13, \mathrm{~A} 14,2, \mathrm{D} 13,0$

$\mathrm{H}, 15, \mathrm{~B} 16,13, \mathrm{~A} 15,2, \mathrm{D} 14,0$ 
C,15,B17,13,A16,2,D15,0

H,18,B18,15,A17,13,D16,0

H, 18,B19,15,A 18,13,D17,0

H,18,B20,15,A 19, 13,D18,0

S, 13,B21,2,A20,1,D19,0

Variables:

$\mathrm{B} 1=1.47164997$

$\mathrm{B} 2=1.8127487$

B3 $=1.09059606$

$\mathrm{B} 4=1.09065052$

B5 $=1.52264395$

B6 $=1.08997662$

$\mathrm{B} 7=1.09157926$

$\mathrm{B} 8=1.52490368$

B9 $=1.08997947$

$\mathrm{B} 10=1.0882988$

B11 $=1.08977984$

$\mathrm{B} 12=2.98421231$

$\mathrm{B} 13=1.08142996$

B14 $=1.48953508$

$\mathrm{B} 15=1.09763044$

$\mathrm{B} 16=1.09286611$

$\mathrm{B} 17=1.52595189$

$\mathrm{B} 18=1.08817761$

$\mathrm{B} 19=1.08922816$

$\mathrm{B} 20=1.08965991$

B21 $=1.70690369$

$\mathrm{A} 1=106.57373174$

$\mathrm{A} 2=107.11471158$

A $3=107.0316348$

A4 $=109.22542124$

A5 $=108.67271765$

$\mathrm{A} 6=109.47567727$

A $7=111.06975245$

A $8=111.05639041$

A9 $=110.91636788$

$\mathrm{A} 10=110.76511182$

A11 $=96.58582581$

A12 $=137.8939466$

A13 $=86.56865844$

A14 $=109.37044431$

A15 $=109.43715218$

A16 $=111.99997143$

A17 $=110.94293436$

A18 $=110.72519093$

A19 $=110.58255073$ 


$$
\begin{aligned}
& \mathrm{A} 20=45.45616744 \\
& \mathrm{D} 1=173.07028591 \\
& \mathrm{D} 2=55.48437353 \\
& \mathrm{D} 3=-65.22706463 \\
& \mathrm{D} 4=56.10553512 \\
& \mathrm{D} 5=-60.61678357 \\
& \text { D6 }=177.65096674 \\
& \mathrm{D} 7=59.74405085 \\
& \text { D8 }=179.91372164 \\
& \text { D9 }=-60.15201005 \\
& \text { D10 }=131.25895937 \\
& \text { D1 }=-36.3650643 \\
& \text { D12 }=96.6623618 \\
& \text { D13 }=-47.13693534 \\
& \text { D14 }=68.38632485 \\
& \text { D15 }=-168.55590706 \\
& \text { D16 }=178.68381102 \\
& \text { D17 }=-60.97232002 \\
& \text { D18 }=58.83265101 \\
& \text { D19 }=-116.05316199
\end{aligned}
$$

\section{$\underline{\text { DPTS••OH (TS5) }}$}

Final structure in terms of initial Z-matrix:

$\mathrm{O}$

$\mathrm{S}, 1, \mathrm{~B} 1$

$\mathrm{C}, 2, \mathrm{~B} 2,1, \mathrm{~A} 1$

H,3,B3, 2,A2, 1,D1,0

$\mathrm{H}, 3, \mathrm{~B} 4,2, \mathrm{~A} 3,1, \mathrm{D} 2,0$

$\mathrm{C}, 3, \mathrm{~B} 5,2, \mathrm{~A} 4,1, \mathrm{D} 3,0$

$\mathrm{H}, 6, \mathrm{~B} 6,3, \mathrm{~A} 5,2, \mathrm{D} 4,0$

H,6,B7,3,A6,2,D5,0

$\mathrm{C}, 6, \mathrm{~B} 8,3, \mathrm{~A} 7,2, \mathrm{D} 6,0$

$\mathrm{H}, 9, \mathrm{~B} 9,6, \mathrm{~A} 8,3, \mathrm{D} 7,0$

$\mathrm{H}, 9, \mathrm{~B} 10,6, \mathrm{~A} 9,3, \mathrm{D} 8,0$

H,9,B11,6,A10,3,D9,0

C,2,B12,1,A11,3,D10,0

H,13,B13,2,A12,1,D11,0

$\mathrm{H}, 13, \mathrm{~B} 14,2, \mathrm{~A} 13,1, \mathrm{D} 12,0$

C,13,B15,2,A14,1,D13,0

$\mathrm{H}, 16, \mathrm{~B} 16,13, \mathrm{~A} 15,2, \mathrm{D} 14,0$

H,16,B17,13,A 16,2,D15,0

C,16,B18,13,A17,2,D16,0

H,19,B19,16,A18,13,D17,0

H,19,B20,16,A19,13,D18,0

H,19,B21,16,A20,13,D19,0 
$\mathrm{S}, 13, \mathrm{~B} 22,2, \mathrm{~A} 21,1, \mathrm{D} 20,0$

$\mathrm{O}, 1, \mathrm{~B} 23,2, \mathrm{~A} 22,3, \mathrm{D} 21,0$

H,2,B24, 1,A23, 24,D22, 0

Variables:

$\mathrm{B} 1=1.47013664$

$\mathrm{B} 2=1.81065074$

B3 $=1.09005346$

B4 $=1.090836$

B5 $=1.52244168$

B6 $=1.08973844$

$\mathrm{B} 7=1.09160341$

$\mathrm{B} 8=1.52483687$

B9 $=1.08988601$

$\mathrm{B} 10=1.08819988$

$\mathrm{B} 11=1.0897914$

$\mathrm{B} 12=2.95404147$

$\mathrm{B} 13=1.0913331$

$\mathrm{B} 14=1.09157715$

$\mathrm{B} 15=1.51327293$

$\mathrm{B} 16=1.16219315$

$\mathrm{B} 17=1.09019154$

$\mathrm{B} 18=1.51503336$

$\mathrm{B} 19=1.08779416$

$\mathrm{B} 20=1.09185507$

B21 $=1.08947007$

$\mathrm{B} 22=1.81739958$

$\mathrm{B} 23=4.45815567$

$\mathrm{B} 24=2.57639107$

$\mathrm{A} 1=106.89020361$

$\mathrm{A} 2=107.06393259$

A $3=106.74636452$

A4 $=109.25861645$

A $5=108.70806608$

A6 $=109.47368761$

$\mathrm{A} 7=110.94961483$

A $8=111.08462815$

A9 $=110.80946785$

$\mathrm{A} 10=110.78578485$

A11 $=99.12375128$

A12 $=70.38893244$

$\mathrm{A} 13=139.01255456$

A14 $=108.48688406$

A15 $=109.44109329$

A16 $=111.01057205$

A17 $=113.07180744$

A18 $=110.82301491$ 

A19 $=110.69535159$
A $20=110.70280278$
$\mathrm{A} 21=44.87470388$
$\mathrm{A} 22=39.12680577$
A23 $=122.9371687$
D1 $=175.85178906$
D2 $=58.33550257$
D3 $=-62.23339059$
D4 $=56.16028316$
D5 $=-60.64265718$
D6 $=177.73731145$
D7 $=59.9110998$
D8 $=-179.97451274$
D9 $=-60.11096248$
D10 $=137.98812914$
D1 $1=34.17380152$
$\mathrm{D} 12=-58.75046872$
D13 $=141.56196145$
D14 $=-14.91463553$
D15 $=99.09454961$
D16 $=-133.24784217$
$\mathrm{D} 17=177.28647964$
D18 $=-62.6290108$
D19 $=57.31172014$
D20 $=-111.88257496$
D21 $=158.17653752$
D22 $=9.72069592$

\section{DPTS••OH (RC5)}

Final structure in terms of initial Z-matrix:

$\mathrm{O}$

$\mathrm{S}, 1, \mathrm{~B} 1$

$\mathrm{C}, 2, \mathrm{~B} 2,1, \mathrm{~A} 1$

$\mathrm{H}, 3, \mathrm{~B} 3,2, \mathrm{~A} 2,1, \mathrm{D} 1,0$

H,3,B4,2,A3,1,D2,0

C,3,B5,2,A4, 1,D3,0

H,6,B6,3,A5,2,D4,0

H,6,B7,3,A6,2,D5,0

$\mathrm{C}, 6, \mathrm{~B} 8,3, \mathrm{~A} 7,2, \mathrm{D} 6,0$

H,9,B9,6,A8,3,D7,0

H,9,B10,6,A9,3,D8,0

H,9,B1 1,6,A10,3,D9,0

C,2,B12,1,A11,3,D10,0

H,13,B13,2,A12,1,D11,0

H,13,B14,2,A13,1,D12,0 


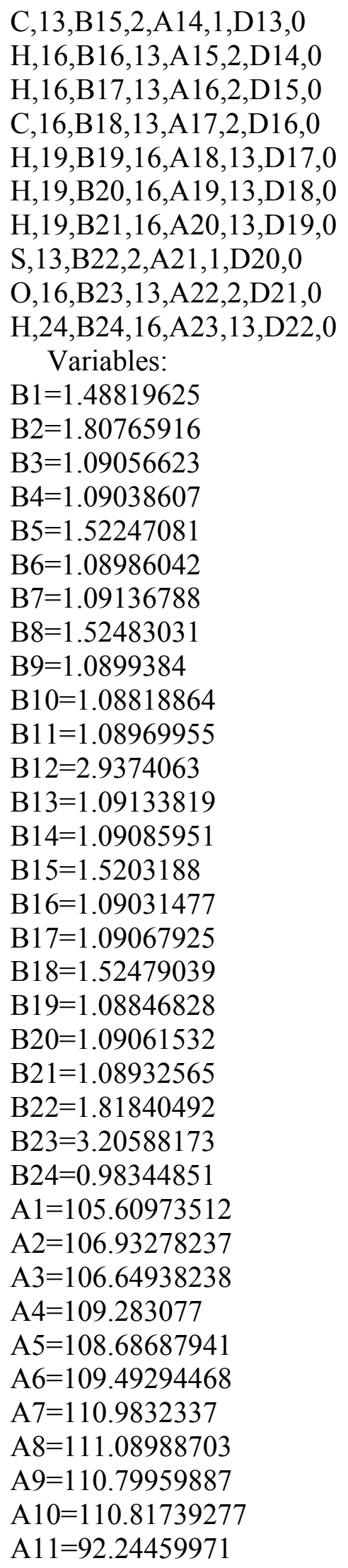



$\mathrm{A} 12=81.05045017$
$\mathrm{A} 13=147.45458805$
A14 $=94.01616976$
A $15=109.38015473$
A16 $=109.06618155$
A17 $=110.67766422$
A $18=110.82302423$
A19 $=111.01626722$
A $20=110.39208423$
$\mathrm{A} 21=45.07885063$
$\mathrm{A} 22=75.24529176$
A23 $=98.4711784$
D1 $=172.46420029$
D2 $=55.02090524$
D3 $=-65.83231458$
D4 $=56.28586627$
D5 $=-60.54379677$
D6 $=177.75603419$
D7 $=59.95876461$
D8 $=-179.96368683$
D9 $=-60.08214385$
D10 $=135.12934066$
$\mathrm{D} 11=8.06376066$
D12 $=-99.51923497$
D13 $=119.37723709$
D14 $=-19.19626548$
D15 $=97.99156579$
D16 $=-139.79704279$
D17 $=-179.61550162$
D18 $=-59.59257517$
D19 $=60.7414344$
D20 $=-118.55765525$
D21 $=-65.30250368$
D22 $=50.3865328$

\section{$\underline{\text { DPTS••OH (PC5) }}$}

Final structure in terms of initial Z-matrix:

$\mathrm{O}$

$\mathrm{S}, 1, \mathrm{~B} 1$

$\mathrm{C}, 2, \mathrm{~B} 2,1, \mathrm{~A} 1$

H,3,B3, 2,A2, 1, D1,0

$\mathrm{H}, 3, \mathrm{~B} 4,2, \mathrm{~A} 3,1, \mathrm{D} 2,0$

$\mathrm{C}, 3, \mathrm{~B} 5,2, \mathrm{~A} 4,1, \mathrm{D} 3,0$

$\mathrm{H}, 6, \mathrm{~B} 6,3, \mathrm{~A} 5,2, \mathrm{D} 4,0$

H,6,B7,3,A6,2,D5,0 
C,6,B8,3,A7,2,D6,0

H,9,B9, 6,A8,3,D7,0

H,9,B10,6,A9,3,D8,0

H,9,B11,6,A10,3,D9, 0

$\mathrm{C}, 2, \mathrm{~B} 12,1, \mathrm{~A} 11,3, \mathrm{D} 10,0$

H,13,B13,2,A12,1,D11,0

H,13,B14,2,A13,1,D12,0

C,13,B15,2,A14,1,D13,0

H,16,B16,13,A15,2,D14,0

H,16,B17,13,A16,2,D15,0

C,16,B18,13,A17,2,D16,0

H,19,B19,16,A 18, 13,D17,0

H,19,B20,16,A19,13,D18,0

H,19,B21,16,A20,13,D19,0

S, 13,B22,2,A21,1,D20,0

$\mathrm{O}, 16, \mathrm{~B} 23,13, \mathrm{~A} 22,2, \mathrm{D} 21,0$

H,24,B24,16,A23,13,D22,0

Variables:

$\mathrm{B} 1=1.47389972$

$\mathrm{B} 2=1.80850495$

$\mathrm{B} 3=1.09033253$

B4 $=1.0901862$

$\mathrm{B} 5=1.52306312$

B6 $=1.09010573$

B7 $=1.09164259$

$\mathrm{B} 8=1.52467939$

B9 $=1.08971377$

$\mathrm{B} 10=1.08836116$

$\mathrm{B} 11=1.0897885$

$\mathrm{B} 12=2.93793586$

$\mathrm{B} 13=1.08851382$

B14 $=1.08931041$

$\mathrm{B} 15=1.47106122$

$\mathrm{B} 16=3.2595088$

B17 $=1.08185982$

$\mathrm{B} 18=1.48853711$

$\mathrm{B} 19=1.09334292$

$\mathrm{B} 20=1.09425537$

$\mathrm{B} 21=1.08984513$

$\mathrm{B} 22=1.86048291$

$\mathrm{B} 23=3.83599713$

B24 $=0.95997234$

$\mathrm{A} 1=107.43657005$

$\mathrm{A} 2=106.3464768$

$\mathrm{A} 3=107.21732013$

A4 $=108.79745532$ 


$$
\begin{aligned}
& \text { A5 }=108.89186069 \\
& \text { A } 6=109.25523764 \\
& \text { A } 7=111.03933636 \\
& \text { A } 8=110.99327287 \\
& \text { A } 9=110.81534128 \\
& \text { A10 }=110.81091838 \\
& \text { A1 }=90.69564769 \\
& \text { A12 }=82.78126674 \\
& \text { A13 }=148.22196677 \\
& \text { A14 }=87.47379017 \\
& \text { A15 }=85.89997693 \\
& \text { A16 }=118.16436009 \\
& \text { A17 }=122.33309032 \\
& \text { A18 }=111.82107576 \\
& \text { A19 }=111.24339347 \\
& \text { A20 }=111.2470434 \\
& \text { A21 }=45.99695208 \\
& \text { A22 }=96.13801146 \\
& \text { A23 }=56.90862851 \\
& \text { D1 }=173.50912566 \\
& \text { D2 }=55.16294746 \\
& \text { D3 }=-65.80845094 \\
& \text { D4 }=57.08433697 \\
& \text { D5 }=-59.81629025 \\
& \text { D6 }=178.75272387 \\
& \text { D7 }=60.13382464 \\
& \text { D8 }=-179.73510591 \\
& \text { D9 }=-59.87513018 \\
& \text { D10 }=136.24612292 \\
& \text { D11 }=0.63673211 \\
& \text { D12 }=-114.23454089 \\
& \text { D13 }=113.55901592 \\
& \text { D14 }=51.99780709 \\
& \text { D15 }=116.00638782 \\
& \text { D16 }=-60.25843768 \\
& \text { D17 }=124.13435087 \\
& \text { D18 }=-117.04548487 \\
& \text { D19 }=3.49581735 \\
& \text { D20 }=-119.64952693 \\
& \text { D21 }=44.75429653 \\
& \text { D22 }=-143.22845052 \\
&
\end{aligned}
$$

\section{$\underline{\mathrm{C}}_{3} \underline{\mathrm{H}}_{7} \underline{\mathrm{S}(=\mathrm{O}) \mathrm{SCH}_{2}} \underline{\mathrm{CHCH}}_{3}$}

Final structure in terms of initial Z-matrix:

$\mathrm{O}$ 


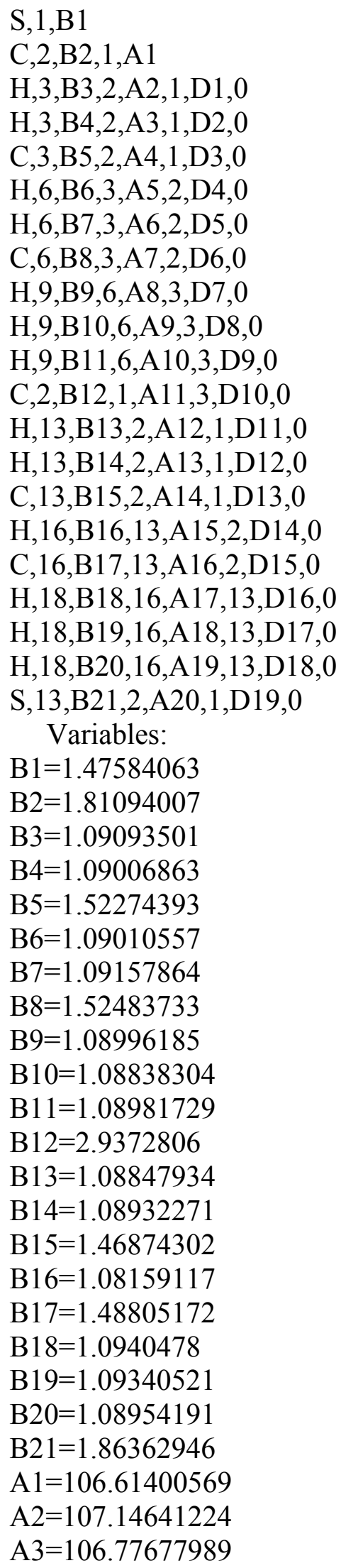




$$
\begin{aligned}
& \text { A4 }=109.16519759 \\
& \text { A5 }=108.66152149 \\
& \mathrm{~A} 6=109.47968209 \\
& \mathrm{~A} 7=111.16173997 \\
& \mathrm{~A} 8=111.06903617 \\
& \text { A9 }=110.88492504 \\
& \text { A10 }=110.78396136 \\
& \text { A } 11=87.74978388 \\
& \mathrm{~A} 12=81.7694351 \\
& \mathrm{~A} 13=147.3568531 \\
& \text { A14 }=88.98163047 \\
& \text { A15 }=118.0355381 \\
& \text { A16 }=122.34792691 \\
& \text { A } 17=111.58858226 \\
& \text { A18 }=111.20962384 \\
& \text { A19 }=111.26116949 \\
& \text { A20 }=45.69463411 \\
& \text { D1 }=171.54134445 \\
& \text { D2 }=53.99996407 \\
& \text { D3 }=-66.83467188 \\
& \text { D4 }=56.43551693 \\
& \text { D5 }=-60.27576102 \\
& \text { D6 }=177.96204916 \\
& \text { D7 }=60.08171067 \\
& \text { D8 }=-179.74247864 \\
& \text { D9 }=-59.84899605 \\
& \text { D10 }=133.87096897 \\
& \mathrm{D} 11=-2.89319579 \\
& \text { D12 }=-115.85880912 \\
& \text { D13 }=109.91107273 \\
& \text { D14 }=115.43512772 \\
& \text { D15 }=-56.09964283 \\
& \text { D16 }=115.78802548 \\
& \text { D17 }=-125.54654429 \\
& \text { D18 }=-4.59722175 \\
& \text { D19=-124.21049994 }
\end{aligned}
$$

\section{DPTS••OH (TS6)}

Final structure in terms of initial Z-matrix:

$\mathrm{O}$

$\mathrm{S}, 1, \mathrm{~B} 1$

$\mathrm{C}, 2, \mathrm{~B} 2,1, \mathrm{~A} 1$

$\mathrm{H}, 3, \mathrm{~B} 3,2, \mathrm{~A} 2,1, \mathrm{D} 1,0$

$\mathrm{H}, 3, \mathrm{~B} 4,2, \mathrm{~A} 3,1, \mathrm{D} 2,0$

C,3,B5,2,A4, 1,D3,0 


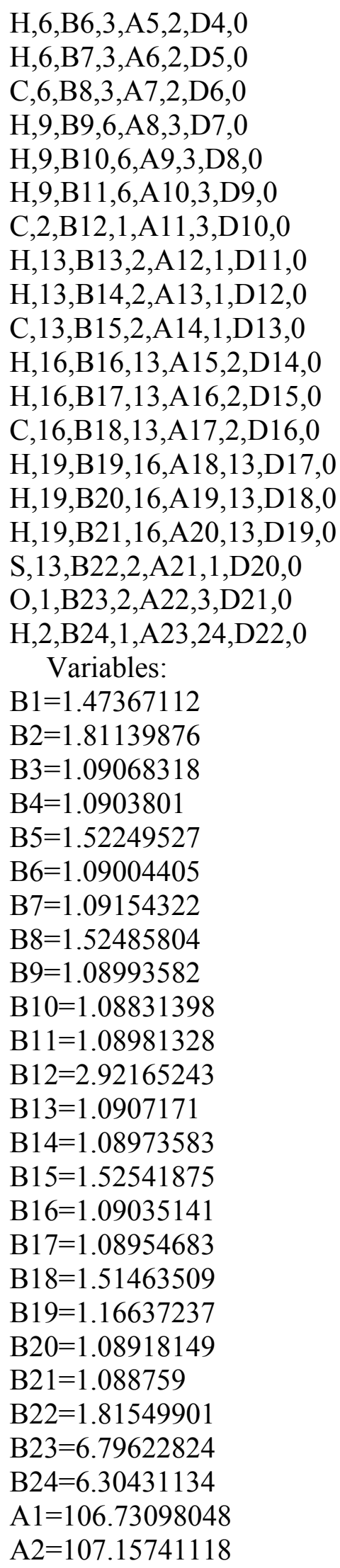



$\mathrm{A} 3=106.75529956$
$\mathrm{A} 4=109.23605886$
A5 $=108.68330096$
$\mathrm{A} 6=109.50513432$
$\mathrm{A} 7=111.07265875$
$\mathrm{A} 8=111.08576637$
A9 $=110.86712218$
A $10=110.7476056$
A11 $=91.68269248$
A12 $=75.91644029$
A13 $=147.35372587$
A14 $=96.83968734$
$\mathrm{A} 15=109.51550307$
A16 $=109.30684198$
$\mathrm{A} 17=110.70524381$
A18 $=108.00388417$
$\mathrm{A} 19=113.50606941$
A20 $=113.04577359$
A21 $=45.99971208$
$\mathrm{A} 22=61.93092411$
A23 $=98.90702293$
$\mathrm{D} 1=172.51067063$
D2 $=54.93674802$
D3 $=-65.75982174$
D4 $=56.11466239$
D5 $=-60.65864172$
D6 $=177.60436935$
D7 $=60.16109329$
D8 $=-179.6513211$
D9 $=-59.7791773$
$\mathrm{D} 10=134.60748848$
D1 $1=12.4138731$
D12 $=-88.03783261$
D13 $=123.16966049$
D14 $=-14.5517948$
D15 $=102.39191304$
D16 $=-135.81118397$
$\mathrm{D} 17=-179.29346732$
D18 $=-63.67005455$
D19 $=62.6561609$
D20 $=-119.60758129$
D21 $=179.30996981$
D22 $=5.35718122$

\section{$\underline{\text { DPTS••OH (RC6) }}$}


Final structure in terms of initial Z-matrix:

$\mathrm{O}$

$\mathrm{S}, 1, \mathrm{~B} 1$

$\mathrm{C}, 2, \mathrm{~B} 2,1, \mathrm{~A} 1$

$\mathrm{H}, 3, \mathrm{~B} 3,2, \mathrm{~A} 2,1, \mathrm{D} 1,0$

$\mathrm{H}, 3, \mathrm{~B} 4,2, \mathrm{~A} 3,1, \mathrm{D} 2,0$

C,3,B5,2,A4,1,D3,0

$\mathrm{H}, 6, \mathrm{~B} 6,3, \mathrm{~A} 5,2, \mathrm{D} 4,0$

H,6,B7,3,A6,2,D5,0

$\mathrm{C}, 6, \mathrm{~B} 8,3, \mathrm{~A} 7,2, \mathrm{D} 6,0$

$\mathrm{H}, 9, \mathrm{~B} 9,6, \mathrm{~A} 8,3, \mathrm{D} 7,0$

H,9,B10,6,A9,3,D8,0

H,9,B11,6,A10,3,D9,0

C,2,B12,1,A11,3,D10,0

$\mathrm{H}, 13, \mathrm{~B} 13,2, \mathrm{~A} 12,1, \mathrm{D} 11,0$

$\mathrm{H}, 13, \mathrm{~B} 14,2, \mathrm{~A} 13,1, \mathrm{D} 12,0$

C,13,B15,2,A14,1,D13,0

$\mathrm{H}, 16, \mathrm{~B} 16,13, \mathrm{~A} 15,2, \mathrm{D} 14,0$

H,16,B17,13,A16,2,D15,0

C,16,B18,13,A17,2,D16,0

H,19,B19,16,A18,13,D17,0

H,19,B20,16,A19,13,D18,0

H,19,B21,16,A20,13,D19,0

S, 13,B22,2,A21,1,D20,0

O,19,B23,16,A22,13,D21,0

H,24,B24,19,A23, 16,D22,0

Variables:

$\mathrm{B} 1=1.48801909$

$\mathrm{B} 2=1.80783755$

$\mathrm{B} 3=1.09053496$

$\mathrm{B} 4=1.09037925$

$\mathrm{B} 5=1.52268911$

B6 $=1.08987582$

B7 $=1.09132174$

$\mathrm{B} 8=1.5247898$

B9 $=1.08996546$

$\mathrm{B} 10=1.08812556$

$\mathrm{B} 11=1.08961527$

$\mathrm{B} 12=2.93876323$

$\mathrm{B} 13=1.09122079$

$\mathrm{B} 14=1.09091529$

B15 $=1.52073504$

$\mathrm{B} 16=1.09011912$

$\mathrm{B} 17=1.09062907$

$\mathrm{B} 18=1.52486847$

$\mathrm{B} 19=1.08849007$ 


$$
\begin{aligned}
& \text { B20 }=1.09064647 \\
& \mathrm{~B} 21=1.08932675 \\
& \mathrm{~B} 22=1.81774788 \\
& \mathrm{~B} 23=3.34452973 \\
& \text { B24 }=0.98337629 \\
& \mathrm{~A} 1=105.63913784 \\
& \mathrm{~A} 2=106.94381008 \\
& \text { A3 }=106.62394667 \\
& \text { A4 }=109.3957491 \\
& \text { A5 }=108.7434489 \\
& \text { A6 }=109.44881789 \\
& \text { A } 7=110.90599212 \\
& \mathrm{~A} 8=111.04711624 \\
& \text { A9 }=110.83827367 \\
& \text { A10 }=110.80573732 \\
& \text { A11 }=92.54225986 \\
& \mathrm{~A} 12=81.02417424 \\
& \text { A13 }=147.39270286 \\
& \text { A14 }=94.21062993 \\
& \text { A15 }=109.41343022 \\
& \text { A16 }=109.09974654 \\
& \text { A17 }=110.64413488 \\
& \mathrm{~A} 18=110.82537593 \\
& \text { A19 }=111.00824721 \\
& \text { A } 20=110.39932876 \\
& \text { A } 21=45.03717489 \\
& \mathrm{~A} 22=71.44748855 \\
& \text { A23 }=124.8969934 \\
& \text { D1 }=172.05867968 \\
& \text { D2 }=54.65427875 \\
& \text { D3 }=-66.25621276 \\
& \text { D4 }=56.74144673 \\
& \text { D5 }=-60.13283339 \\
& \text { D6 }=178.22180785 \\
& \text { D7 }=59.87113125 \\
& \text { D8 }=179.96188934 \\
& \text { D9 }=-60.1461924 \\
& \text { D10 }=135.16133164 \\
& \text { D1 } 1=8.49650064 \\
& \text { D12 }=-99.03269145 \\
& \mathrm{D} 13=119.75992536 \\
& \text { D14 }=-19.43735171 \\
& \text { D15 }=97.78684245 \\
& \text { D16 }=-140.00470985 \\
& \text { D17 }=-179.81256567 \\
& \text { D18 }=-59.77990815
\end{aligned}
$$




$$
\begin{aligned}
& \text { D19 }=60.54423789 \\
& \text { D20 }=-118.27717232 \\
& \text { D21 }=70.56278929 \\
& \text { D22 }=-19.11334799
\end{aligned}
$$

\section{DPTS••OH (PC6)}

Final structure in terms of initial Z-matrix:

$\mathrm{O}$

$\mathrm{S}, 1, \mathrm{~B} 1$

$\mathrm{C}, 2, \mathrm{~B} 2,1, \mathrm{~A} 1$

$\mathrm{H}, 3, \mathrm{~B} 3,2, \mathrm{~A} 2,1, \mathrm{D} 1,0$

H,3,B4,2,A3, 1,D2,0

C,3,B5,2,A4, 1,D3,0

$\mathrm{H}, 6, \mathrm{~B} 6,3, \mathrm{~A} 5,2, \mathrm{D} 4,0$

$\mathrm{H}, 6, \mathrm{~B} 7,3, \mathrm{~A} 6,2, \mathrm{D} 5,0$

$\mathrm{C}, 6, \mathrm{~B} 8,3, \mathrm{~A} 7,2, \mathrm{D} 6,0$

$\mathrm{H}, 9, \mathrm{~B} 9,6, \mathrm{~A} 8,3, \mathrm{D} 7,0$

H,9,B10,6,A9,3,D8,0

H,9,B11,6,A10,3,D9,0

C,2,B12,1,A11,3,D10,0

$\mathrm{H}, 13, \mathrm{~B} 13,2, \mathrm{~A} 12,1, \mathrm{D} 11,0$

H,13,B14,2,A13,1,D12,0

C,13,B15,2,A14,1,D13,0

$\mathrm{H}, 16, \mathrm{~B} 16,13, \mathrm{~A} 15,2, \mathrm{D} 14,0$

H,16,B17,13,A16,2,D15,0

C,16,B18,13,A17,2,D16,0

H,19,B19,16,A18,13,D17,0

H,19,B20,16,A19,13,D18,0

H,19,B21,16,A20,13,D19,0

S, 13,B22,2,A21,1,D20,0

O,19,B23,16,A22,13,D21,0

H,24,B24,19,A23, 16,D22,0

Variables:

$\mathrm{B} 1=1.47376457$

$\mathrm{B} 2=1.81158386$

$\mathrm{B} 3=1.09074141$

B4 $=1.09034997$

$\mathrm{B} 5=1.52261497$

$\mathrm{B} 6=1.09000723$

$\mathrm{B} 7=1.09153076$

$\mathrm{B} 8=1.52474227$

$\mathrm{B} 9=1.08992172$

$\mathrm{B} 10=1.08832657$

$\mathrm{B} 11=1.08979004$

$\mathrm{B} 12=2.92183932$ 


$$
\begin{aligned}
& B 13=1.09019003 \\
& B 14=1.08909772 \\
& B 15=1.53594442 \\
& B 16=1.09076753 \\
& B 17=1.09001371 \\
& B 18=1.48864196 \\
& B 19=2.33351357 \\
& B 20=1.08075418 \\
& B 21=1.08055736 \\
& B 22=1.81547015 \\
& B 23=3.175212 \\
& B 24=0.95877505 \\
& A 1=106.67633083 \\
& A 2=107.17667387 \\
& A 3=106.76604405 \\
& A 4=109.06632486 \\
& A 5=108.60650455 \\
& A 6=109.47876366 \\
& A 7=111.15702956 \\
& A 8=111.11804574 \\
& A 9=110.81814681 \\
& A 10=110.75922205 \\
& A 11=91.82889254 \\
& A 12=75.7115533 \\
& A 13=147.3999848 \\
& A 14=97.11257573 \\
& A 15=108.95412488 \\
& A 16=108.92216363 \\
& A 17=110.60343888 \\
& A 18=85.65448622 \\
& A 19=120.21018736 \\
& A 20=120.19193782 \\
& A 21=45.98916379 \\
& A 22=76.13533289 \\
& A 23=129.81169534 \\
& D 1=173.10127363 \\
& D 2=55.4390375 \\
& D 3=-65.16306677 \\
& D 4=55.84115608 \\
& D 5=-60.89551819 \\
& D 6=177.31967392 \\
& D 7=60.11790061 \\
& D=-90153709 \\
& D
\end{aligned}
$$




$$
\begin{aligned}
& \text { D12 }=-87.04594977 \\
& \text { D13 }=123.90257196 \\
& \text { D14 }=-13.88766362 \\
& \text { D15 }=102.51291201 \\
& \text { D16 }=-135.37393867 \\
& \text { D17 }=-175.42959161 \\
& \text { D18 }=-81.51675783 \\
& \text { D19 }=81.6409227 \\
& \text { D20 }=-119.39483944 \\
& \text { D21 }=-172.8968331 \\
& \text { D22 }=-170.02364347
\end{aligned}
$$

\section{$\underline{\mathrm{C}}_{3} \underline{\mathrm{H}}_{7} \underline{\mathrm{S}(=\mathrm{O}) \mathrm{SCH}_{2}} \underline{\mathrm{CH}}_{2} \underline{\mathrm{CH}_{2}}$}

Final structure in terms of initial Z-matrix:

$\mathrm{O}$

$\mathrm{S}, 1, \mathrm{~B} 1$

$\mathrm{C}, 2, \mathrm{~B} 2,1, \mathrm{~A} 1$

$\mathrm{H}, 3, \mathrm{~B} 3,2, \mathrm{~A} 2,1, \mathrm{D} 1,0$

$\mathrm{H}, 3, \mathrm{~B} 4,2, \mathrm{~A} 3,1, \mathrm{D} 2,0$

$\mathrm{C}, 3, \mathrm{~B} 5,2, \mathrm{~A} 4,1, \mathrm{D} 3,0$

$\mathrm{H}, 6, \mathrm{~B} 6,3, \mathrm{~A} 5,2, \mathrm{D} 4,0$

$\mathrm{H}, 6, \mathrm{~B} 7,3, \mathrm{~A} 6,2, \mathrm{D} 5,0$

$\mathrm{C}, 6, \mathrm{~B} 8,3, \mathrm{~A} 7,2, \mathrm{D} 6,0$

$\mathrm{H}, 9, \mathrm{~B} 9,6, \mathrm{~A} 8,3, \mathrm{D} 7,0$

$\mathrm{H}, 9, \mathrm{~B} 10,6, \mathrm{~A} 9,3, \mathrm{D} 8,0$

$\mathrm{H}, 9, \mathrm{~B} 11,6, \mathrm{~A} 10,3, \mathrm{D} 9,0$

$\mathrm{C}, 2, \mathrm{~B} 12,1, \mathrm{~A} 11,3, \mathrm{D} 10,0$

$\mathrm{H}, 13, \mathrm{~B} 13,2, \mathrm{~A} 12,1, \mathrm{D} 11,0$

$\mathrm{H}, 13, \mathrm{~B} 14,2, \mathrm{~A} 13,1, \mathrm{D} 12,0$

$\mathrm{C}, 13, \mathrm{~B} 15,2, \mathrm{~A} 14,1, \mathrm{D} 13,0$

$\mathrm{H}, 16, \mathrm{~B} 16,13, \mathrm{~A} 15,2, \mathrm{D} 14,0$

$\mathrm{H}, 16, \mathrm{~B} 17,13, \mathrm{~A} 16,2, \mathrm{D} 15,0$

C,16,B18,13,A17,2,D16,0

H,19,B19,16,A18,13,D17,0

H,19,B20,16,A19,13,D18,0

S, 13,B21,2,A20,1,D19,0

Variables:

B1 $=1.47344992$

$\mathrm{B} 2=1.81171697$

B3 $=1.09060188$

B4 $=1.09038001$

$\mathrm{B} 5=1.52247422$

$\mathrm{B} 6=1.09004851$

B7 $=1.09158655$

$\mathrm{B} 8=1.52484763$ 


$$
\begin{aligned}
& \text { B9 }=1.08999292 \\
& \mathrm{~B} 10=1.08834396 \\
& \mathrm{~B} 11=1.08982691 \\
& \mathrm{~B} 12=2.92408118 \\
& \mathrm{~B} 13=1.09000059 \\
& \text { B14=1.08907713 } \\
& \mathrm{B} 15=1.53617225 \\
& \mathrm{~B} 16=1.09206617 \\
& \mathrm{~B} 17=1.0896851 \\
& \mathrm{~B} 18=1.48625407 \\
& \mathrm{~B} 19=1.07893455 \\
& \mathrm{~B} 20=1.07958008 \\
& \text { B21 }=1.81698521 \\
& \mathrm{~A} 1=106.65372826 \\
& \mathrm{~A} 2=107.19451048 \\
& \mathrm{~A} 3=106.75562028 \\
& \text { A4=109.16740794 } \\
& \text { A5 }=108.66825662 \\
& \text { A } 6=109.4809519 \\
& \text { A } 7=111.12091207 \\
& \mathrm{~A} 8=111.08987366 \\
& \text { A9 }=110.86664709 \\
& \mathrm{~A} 10=110.75630646 \\
& \text { A11 }=92.37791334 \\
& \mathrm{~A} 12=76.21093445 \\
& \text { A13 }=147.62238186 \\
& \text { A14 }=96.77174981 \\
& \text { A } 15=108.29802077 \\
& \text { A16 }=108.54191756 \\
& \text { A17 }=111.03036109 \\
& \mathrm{~A} 18=121.02419246 \\
& \text { A19 }=120.26086767 \\
& \text { A20 }=45.91006875 \\
& \mathrm{D} 1=172.84081548 \\
& \text { D2 }=55.24609255 \\
& \text { D3 }=-65.41940699 \\
& \text { D4 }=56.09159116 \\
& \text { D5 }=-60.64462 \\
& \text { D6 }=177.61906165 \\
& \text { D7 }=60.04574073 \\
& \text { D8 }=-179.76973774 \\
& \text { D9 }=-59.90548234 \\
& \text { D10 }=135.01378857 \\
& \text { D1 } 1=13.31411293 \\
& \text { D12 }=-87.81949047 \\
& \mathrm{D} 13=123.76916998
\end{aligned}
$$




$$
\begin{aligned}
& \text { D14 }=-14.16196358 \\
& \text { D15 }=102.03146941 \\
& \text { D16 }=-135.58532549 \\
& \text { D17 }=-93.29395376 \\
& \text { D18 }=76.95052522 \\
& \text { D19 }=-118.91603082
\end{aligned}
$$

\section{$\underline{\text { DPTS・・OH (TS7) }}$}

Final structure in terms of initial Z-matrix:

$\mathrm{O}$

$\mathrm{S}, 1, \mathrm{~B} 1$

$\mathrm{C}, 2, \mathrm{~B} 2,1, \mathrm{~A} 1$

H,3,B3, 2,A2, 1, D1,0

$\mathrm{H}, 3, \mathrm{~B} 4,2, \mathrm{~A} 3,1, \mathrm{D} 2,0$

C,3,B5,2,A4,1,D3,0

$\mathrm{H}, 6, \mathrm{~B} 6,3, \mathrm{~A} 5,2, \mathrm{D} 4,0$

H,6,B7,3,A6,2,D5,0

$\mathrm{C}, 6, \mathrm{~B} 8,3, \mathrm{~A} 7,2, \mathrm{D} 6,0$

H,9,B9, 6,A8,3,D7,0

$\mathrm{H}, 9, \mathrm{~B} 10,6, \mathrm{~A} 9,3, \mathrm{D} 8,0$

H,9,B11,6,A10,3,D9,0

C,2,B12,1,A11,3,D10,0

$\mathrm{H}, 13, \mathrm{~B} 13,2, \mathrm{~A} 12,1, \mathrm{D} 11,0$

$\mathrm{H}, 13, \mathrm{~B} 14,2, \mathrm{~A} 13,1, \mathrm{D} 12,0$

C,13,B15,2,A14,1,D13,0

H,16,B16,13,A15,2,D14,0

$\mathrm{H}, 16, \mathrm{~B} 17,13, \mathrm{~A} 16,2, \mathrm{D} 15,0$

C,16,B18,13,A17,2,D16,0

$\mathrm{H}, 19, \mathrm{~B} 19,16, \mathrm{~A} 18,13, \mathrm{D} 17,0$

H,19,B20,16,A19,13,D18,0

H,19,B21,16,A20,13,D19,0

S, 13,B22,2,A21,1,D20,0

$\mathrm{O}, 1, \mathrm{~B} 23,2, \mathrm{~A} 22,3, \mathrm{D} 21,0$

H,2,B24, 1,A23,24,D22,0

Variables:

$\mathrm{B} 1=1.48307891$

$\mathrm{B} 2=1.8109473$

$\mathrm{B} 3=1.09074104$

B4 $=1.09107149$

$\mathrm{B} 5=1.5225136$

$\mathrm{B} 6=1.08990226$

$\mathrm{B} 7=1.09171056$

$\mathrm{B} 8=1.52493153$

B9 $=1.08989198$

$\mathrm{B} 10=1.08827923$ 


$$
\begin{aligned}
& \mathrm{B} 11=1.0897151 \\
& \mathrm{~B} 12=3.04531216 \\
& \mathrm{~B} 13=1.09143448 \\
& \text { B14 }=1.08924665 \\
& \mathrm{~B} 15=1.52071395 \\
& \mathrm{~B} 16=1.09071873 \\
& \mathrm{~B} 17=1.09088935 \\
& \mathrm{~B} 18=1.5255826 \\
& \mathrm{~B} 19=1.08826219 \\
& \text { B20 }=1.08984449 \\
& \text { B21 }=1.08991428 \\
& \text { B22 }=1.80410243 \\
& \mathrm{~B} 23=2.78296771 \\
& \text { B24 }=2.50463942 \\
& \mathrm{~A} 1=105.6403099 \\
& \text { A2 }=106.8851771 \\
& \mathrm{~A} 3=106.30254882 \\
& \mathrm{~A} 4=109.62580845 \\
& \mathrm{~A} 5=108.70682008 \\
& \text { A6 }=109.54320718 \\
& \text { A7 }=110.9699366 \\
& \mathrm{~A} 8=111.02575919 \\
& \text { A9 }=110.89686887 \\
& \mathrm{~A} 10=110.77240384 \\
& \text { A11 }=99.15915753 \\
& \mathrm{~A} 12=67.82182518 \\
& \mathrm{~A} 13=139.47391136 \\
& \text { A14 }=106.24961327 \\
& \text { A15 }=109.17385472 \\
& \text { A16 }=109.32274587 \\
& \text { A17 }=110.96594437 \\
& \mathrm{~A} 18=110.95760393 \\
& \text { A } 19=110.88620849 \\
& \mathrm{~A} 20=110.89402311 \\
& \text { A21 }=45.98394731 \\
& \text { A22 }=76.95942184 \\
& \text { A23 }=52.84979579 \\
& \text { D1 }=172.36100325 \\
& \mathrm{D} 2=54.99292083 \\
& \text { D3 }=-65.84334308 \\
& \text { D4 }=56.69354771 \\
& \text { D5 }=-60.13476191 \\
& \text { D6 }=178.14516488 \\
& \text { D7 }=60.11534545 \\
& \text { D8 }=-179.73971423 \\
& \text { D9 }=-59.77964293
\end{aligned}
$$



$\mathrm{D} 10=134.11363156$
D1 $1=31.92680245$
D12 $=-58.57805508$
$\mathrm{D} 13=139.56238613$
D14 $=-12.40516075$
D15 $=104.0748926$
D16 $=-134.22388891$
D17 $=-179.99932594$
D18 $=-59.93228221$
$\mathrm{D} 19=59.8846211$
D20 $=-113.99253476$
D2 $1=-85.55493532$
D22 $=1.60833498$

\section{DPTS••OH (RC7)}

Final structure in terms of initial Z-matrix:

$\mathrm{O}$

$\mathrm{S}, 1, \mathrm{~B} 1$

$\mathrm{C}, 2, \mathrm{~B} 2,1, \mathrm{~A} 1$

$\mathrm{H}, 3, \mathrm{~B} 3,2, \mathrm{~A} 2,1, \mathrm{D} 1,0$

$\mathrm{H}, 3, \mathrm{~B} 4,2, \mathrm{~A} 3,1, \mathrm{D} 2,0$

C,3,B5,2,A4, 1,D3,0

$\mathrm{H}, 6, \mathrm{~B} 6,3, \mathrm{~A} 5,2, \mathrm{D} 4,0$

$\mathrm{H}, 6, \mathrm{~B} 7,3, \mathrm{~A} 6,2, \mathrm{D} 5,0$

$\mathrm{C}, 6, \mathrm{~B} 8,3, \mathrm{~A} 7,2, \mathrm{D} 6,0$

$\mathrm{H}, 9, \mathrm{~B} 9,6, \mathrm{~A} 8,3, \mathrm{D} 7,0$

H,9,B10,6,A9,3,D8,0

H,9,B11,6,A10,3,D9,0

C,2,B12,1,A11,3,D10,0

H,13,B13,2,A12,1,D11,0

H,13,B14,2,A13,1,D12,0

C,13,B15,2,A14,1,D13,0

H,16,B16,13,A15,2,D14,0

H,16,B17,13,A16,2,D15,0

C,16,B18,13,A17,2,D16,0

H,19,B19,16,A18,13,D17,0

H,19,B20,16,A19,13,D18,0

H,19,B21,16,A20,13,D19,0

S, 13,B22,2,A21,1,D20,0

O,23,B23,13,A22,2,D21,0

H,24,B24,23,A23, 13,D22,0

Variables:

$\mathrm{B} 1=1.4857657$

$\mathrm{B} 2=1.81036709$

$\mathrm{B} 3=1.09104612$ 


$$
\begin{aligned}
& B 4=1.09110986 \\
& B 5=1.52264592 \\
& B 6=1.08987882 \\
& B 7=1.09136062 \\
& B 8=1.52506772 \\
& B 9=1.08983157 \\
& B 10=1.08814221 \\
& B 11=1.08946197 \\
& B 12=2.93190705 \\
& B 13=1.09043398 \\
& B 14=1.09019056 \\
& B 15=1.52084605 \\
& B 16=1.09108889 \\
& B 17=1.0901897 \\
& B 18=1.52520114 \\
& B 19=1.08831316 \\
& B 20=1.09007478 \\
& B 21=1.08970071 \\
& B 22=1.81392788 \\
& B 23=2.84539646 \\
& B 24=0.97916078 \\
& A 1=106.21887968 \\
& A 2=106.93868127 \\
& A 3=106.25326499 \\
& A 4=109.397715 \\
& A 5=108.72186148 \\
& A 6=109.63235311 \\
& A 7=110.89231611 \\
& A 8=111.04665578 \\
& A 9=110.83116537 \\
& A 10=110.74170196 \\
& A 11=87.20706406 \\
& A 12=77.65715707 \\
& A 13=147.04618543 \\
& A 14=95.89343857 \\
& A 15=109.38121752 \\
& A 16=109.22269525 \\
& A 17=110.97538849 \\
& A 18=111.04599265 \\
& A 19=110.90101471 \\
& A 20=110.84136494 \\
& A 21=45.62630154 \\
& A 22=91.24486064 \\
& A 23=79.58953879 \\
& D 1=171.60393938 \\
& D 2=54.3530939 \\
& \text { a }
\end{aligned}
$$




$$
\begin{aligned}
& D 3=-66.69492527 \\
& D 4=56.51375193 \\
& D 5=-60.40733163 \\
& D 6=177.84275879 \\
& D 7=60.25870017 \\
& D 8=-179.61797839 \\
& D 9=-59.68991226 \\
& D 10=131.63302442 \\
& D 11=5.18948267 \\
& D 12=-97.39737595 \\
& D 13=116.3692254 \\
& D 14=-16.42628484 \\
& D 15=100.16661235 \\
& D 16=-137.76122915 \\
& D 17=179.90069379 \\
& D 18=-60.00422663 \\
& D 19=59.81846809 \\
& D 20=-124.34787242 \\
& D 21=83.17579174 \\
& D 22=-74.72955692
\end{aligned}
$$

\section{$\underline{\text { DPTS・・OH (PC7) }}$}

Final structure in terms of initial Z-matrix:

$\mathrm{O}$

$\mathrm{S}, 1, \mathrm{~B} 1$

$\mathrm{C}, 2, \mathrm{~B} 2,1, \mathrm{~A} 1$

H,3,B3, 2,A2, 1,D1,0

$\mathrm{H}, 3, \mathrm{~B} 4,2, \mathrm{~A} 3,1, \mathrm{D} 2,0$

$\mathrm{C}, 3, \mathrm{~B} 5,2, \mathrm{~A} 4,1, \mathrm{D} 3,0$

$\mathrm{H}, 6, \mathrm{~B} 6,3, \mathrm{~A} 5,2, \mathrm{D} 4,0$

H,6,B7,3,A6,2,D5,0

$\mathrm{C}, 6, \mathrm{~B} 8,3, \mathrm{~A} 7,2, \mathrm{D} 6,0$

$\mathrm{H}, 9, \mathrm{~B} 9,6, \mathrm{~A} 8,3, \mathrm{D} 7,0$

$\mathrm{H}, 9, \mathrm{~B} 10,6, \mathrm{~A} 9,3, \mathrm{D} 8,0$

H,9,B11,6,A10,3,D9,0

C,2,B12,1,A11,3,D10,0

$\mathrm{H}, 13, \mathrm{~B} 13,2, \mathrm{~A} 12,1, \mathrm{D} 11,0$

$\mathrm{H}, 13, \mathrm{~B} 14,2, \mathrm{~A} 13,1, \mathrm{D} 12,0$

$\mathrm{C}, 13, \mathrm{~B} 15,2, \mathrm{~A} 14,1, \mathrm{D} 13,0$

$\mathrm{H}, 16, \mathrm{~B} 16,13, \mathrm{~A} 15,2, \mathrm{D} 14,0$

H,16,B17,13,A16,2,D15,0

C,16,B18,13,A17,2,D16,0

$\mathrm{H}, 19, \mathrm{~B} 19,16, \mathrm{~A} 18,13, \mathrm{D} 17,0$

H,19,B20,16,A19,13,D18,0

H,19,B21,16,A20,13,D19,0 
S, 13,B22,2,A21,1,D20,0

$\mathrm{O}, 23, \mathrm{~B} 23,13, \mathrm{~A} 22,2, \mathrm{D} 21,0$

$\mathrm{H}, 24, \mathrm{~B} 24,23, \mathrm{~A} 23,13, \mathrm{D} 22,0$

Variables:

B1 $=1.49937242$

$\mathrm{B} 2=1.7990694$

B3 $=1.09169704$

B4 $=1.09127973$

$\mathrm{B} 5=1.52499303$

B6 $=1.08991815$

$\mathrm{B} 7=1.09093296$

$\mathrm{B} 8=1.5246424$

$\mathrm{B} 9=1.08996449$

$\mathrm{B} 10=1.08831449$

B11 $=1.08958752$

$\mathrm{B} 12=3.79617806$

$\mathrm{B} 13=1.09163105$

$\mathrm{B} 14=1.08972766$

$\mathrm{B} 15=1.52384252$

B16 $=1.09146604$

B17 $=1.09156062$

$\mathrm{B} 18=1.52518563$

$\mathrm{B} 19=1.08854628$

$\mathrm{B} 20=1.08966664$

B21 $=1.0897851$

$\mathrm{B} 22=1.79911297$

$\mathrm{B} 23=1.64609563$

B24 $=0.97459861$

$\mathrm{A} 1=105.35747627$

$\mathrm{A} 2=105.63376594$

A $3=106.7574183$

A4 $=112.10931006$

A $5=108.50504368$

$\mathrm{A} 6=109.44284016$

A7 $=111.15485646$

A $8=111.06113583$

A9 $=110.8773858$

A10 $=110.74016275$

A11 $=67.19198194$

A12 $=49.80599482$

A13 $=142.90000064$

A14 $=105.41842356$

A15 $=109.22252497$

A16 $=109.513682$

A17 $=111.37535266$

A18 $=111.12331933$ 


$$
\begin{aligned}
& \text { A19 }=110.82390442 \\
& \text { A20 }=110.77721724 \\
& \text { A2 } 1=63.43381698 \\
& \text { A22 }=100.93558051 \\
& \text { A23 }=107.96128817 \\
& \text { D1 }=177.47645784 \\
& \text { D2 }=62.16248477 \\
& \text { D3 }=-60.17020037 \\
& \text { D4 }=55.35329861 \\
& \text { D5 }=-61.18423699 \\
& \text { D6 }=176.74980596 \\
& \text { D7 }=59.94173254 \\
& \text { D8 }=-179.94976919 \\
& \text { D9 }=-60.00738431 \\
& \text { D10 }=106.47158355 \\
& \text { D1 } 1=52.69177411 \\
& \text { D12 }=-12.44162599 \\
& \text { D13 }=157.06153612 \\
& \text { D14 }=7.10474883 \\
& \text { D15 }=123.8927691 \\
& \text { D16 }=-114.4711235 \\
& \text { D17 }=179.5946524 \\
& \text { D18 }=-60.27040752 \\
& \text { D19 }=59.45313915 \\
& \text { D20 }=-98.68640969 \\
& \text { D21 }=78.36005544 \\
& \text { D22 }=-72.06351217
\end{aligned}
$$

\section{$\underline{\mathrm{CH}}_{3} \underline{\mathrm{CH}}_{2} \underline{\mathrm{CH}} 2$}

Final structure in terms of initial Z-matrix:

C

$\mathrm{H}, 1, \mathrm{~B} 1$

$\mathrm{H}, 1, \mathrm{~B} 2,2, \mathrm{~A} 1$

$\mathrm{C}, 1, \mathrm{~B} 3,2, \mathrm{~A} 2,3, \mathrm{D} 1,0$

$\mathrm{H}, 4, \mathrm{~B} 4,1, \mathrm{~A} 3,2, \mathrm{D} 2,0$

$\mathrm{H}, 4, \mathrm{~B} 5,1, \mathrm{~A} 4,2, \mathrm{D} 3,0$

$\mathrm{C}, 4, \mathrm{~B} 6,1, \mathrm{~A} 5,2, \mathrm{D} 4,0$

$\mathrm{H}, 7, \mathrm{~B} 7,4, \mathrm{~A} 6,1, \mathrm{D} 5,0$

H,7,B8,4,A7,1,D6,0

H, 7,B9, 4,A8, 1,D7,0

S, 1,B10,4,A9,7,D8,0

O, 11,B11,1,A10,4,D9,0

H,12,B12,11,A11,1,D10,0

Variables:

$\mathrm{B} 1=1.09088253$ 


$$
\begin{aligned}
& \mathrm{B} 2=1.0934365 \\
& \mathrm{~B} 3=1.52133161 \\
& \mathrm{~B} 4=1.09164481 \\
& \mathrm{~B} 5=1.09069882 \\
& \mathrm{~B} 6=1.52512144 \\
& \mathrm{~B} 7=1.08858905 \\
& \mathrm{~B} 8=1.09028542 \\
& \mathrm{~B} 9=1.0899844 \\
& \mathrm{~B} 10=1.79433603 \\
& \mathrm{~B} 11=1.65954984 \\
& \mathrm{~B} 12=0.96118565 \\
& \mathrm{~A} 1=107.502774 \\
& \mathrm{~A} 2=111.13576418 \\
& \mathrm{~A} 3=108.93077269 \\
& \mathrm{~A} 4=109.00046068 \\
& \mathrm{~A} 5=111.67139523 \\
& \mathrm{~A} 6=111.26135118 \\
& \mathrm{~A} 7=110.81402788 \\
& \mathrm{~A} 8=110.90731421 \\
& \mathrm{~A} 9=114.78918704 \\
& \mathrm{~A} 10=100.33061264 \\
& \mathrm{~A} 11=108.50462676 \\
& \mathrm{D} 1=121.44899272 \\
& \mathrm{D} 2=63.98924653 \\
& \mathrm{D} 3=-179.41130045 \\
& \mathrm{D} 4=-57.18133229 \\
& \mathrm{D} 5=-179.93269567 \\
& \mathrm{D} 6=-59.82424909 \\
& \mathrm{D} 7=59.86871818 \\
& \mathrm{D} 8=179.6231391 \\
& \mathrm{D} 9=67.60646854 \\
& \mathrm{D} 10=-92.37697035
\end{aligned}
$$

\section{$\underline{\mathrm{CH}}_{3} \underline{\mathrm{CH}}_{2} \underline{\mathrm{CH}} 2 \underline{2} \underline{\mathrm{S}(=\mathrm{O})}$}

Final structure in terms of initial Z-matrix:

$\mathrm{O}$

$\mathrm{S}, 1, \mathrm{~B} 1$

$\mathrm{C}, 2, \mathrm{~B} 2,1, \mathrm{~A} 1$

$\mathrm{H}, 3, \mathrm{~B} 3,2, \mathrm{~A} 2,1, \mathrm{D} 1,0$

$\mathrm{H}, 3, \mathrm{~B} 4,2, \mathrm{~A} 3,1, \mathrm{D} 2,0$

$\mathrm{C}, 3, \mathrm{~B} 5,2, \mathrm{~A} 4,1, \mathrm{D} 3,0$

$\mathrm{H}, 6, \mathrm{~B} 6,3, \mathrm{~A} 5,2, \mathrm{D} 4,0$

$\mathrm{H}, 6, \mathrm{~B} 7,3, \mathrm{~A} 6,2, \mathrm{D} 5,0$

$\mathrm{C}, 6, \mathrm{~B} 8,3, \mathrm{~A} 7,2, \mathrm{D} 6,0$

$\mathrm{H}, 9, \mathrm{~B} 9,6, \mathrm{~A} 8,3, \mathrm{D} 7,0$ 


$$
\begin{aligned}
& \mathrm{H}, 9, \mathrm{~B} 10,6, \mathrm{~A} 9,3, \mathrm{D} 8,0 \\
& \mathrm{H}, 9, \mathrm{~B} 11,6, \mathrm{~A} 10,3, \mathrm{D} 9,0 \\
& \text { Variables: } \\
& \mathrm{B} 1=1.49157439 \\
& \mathrm{~B} 2=1.80290071 \\
& \mathrm{~B} 3=1.09193264 \\
& \mathrm{~B} 4=1.09140159 \\
& \mathrm{~B} 5=1.52411736 \\
& \mathrm{~B} 6=1.08985354 \\
& \mathrm{~B} 7=1.09110157 \\
& \mathrm{~B} 8=1.52442867 \\
& \mathrm{~B} 9=1.09012085 \\
& \mathrm{~B} 10=1.08846761 \\
& \mathrm{~B} 11=1.08987246 \\
& \mathrm{~A} 1=105.64937908 \\
& \mathrm{~A} 2=105.73599856 \\
& \mathrm{~A} 3=107.09170475 \\
& \mathrm{~A} 4=112.20583128 \\
& \mathrm{~A} 5=108.40670542 \\
& \mathrm{~A} 6=109.17566555 \\
& \mathrm{~A} 7=111.50546936 \\
& \mathrm{~A} 8=111.07667345 \\
& \mathrm{~A} 9=110.922662 \\
& \mathrm{~A} 10=110.82724807 \\
& \mathrm{D} 1=178.36371962 \\
& \mathrm{D} 2=62.79541403 \\
& \mathrm{D} 3=-59.37843064 \\
& \mathrm{D} 4=55.33285431 \\
& \mathrm{D} 5=-60.94341615 \\
& \mathrm{D} 6=177.0675485 \\
& \mathrm{D} 7=59.66123359 \\
& \mathrm{D} 8=179.77601295 \\
& \mathrm{D} 9=-60.28607766 \\
&
\end{aligned}
$$

\section{$\underline{\text { DPTS } \bullet \text { OH (TS8) }}$}

Final structure in terms of initial Z-matrix:

$\mathrm{O}$

$\mathrm{S}, 1, \mathrm{~B} 1$

$\mathrm{C}, 2, \mathrm{~B} 2,1, \mathrm{~A} 1$

$\mathrm{H}, 3, \mathrm{~B} 3,2, \mathrm{~A} 2,1, \mathrm{D} 1,0$

H,3,B4, 2,A3, 1, D2,0

$\mathrm{C}, 3, \mathrm{~B} 5,2, \mathrm{~A} 4,1, \mathrm{D} 3,0$

$\mathrm{H}, 6, \mathrm{~B} 6,3, \mathrm{~A} 5,2, \mathrm{D} 4,0$

$\mathrm{H}, 6, \mathrm{~B} 7,3, \mathrm{~A} 6,2, \mathrm{D} 5,0$

$\mathrm{C}, 6, \mathrm{~B} 8,3, \mathrm{~A} 7,2, \mathrm{D} 6,0$ 


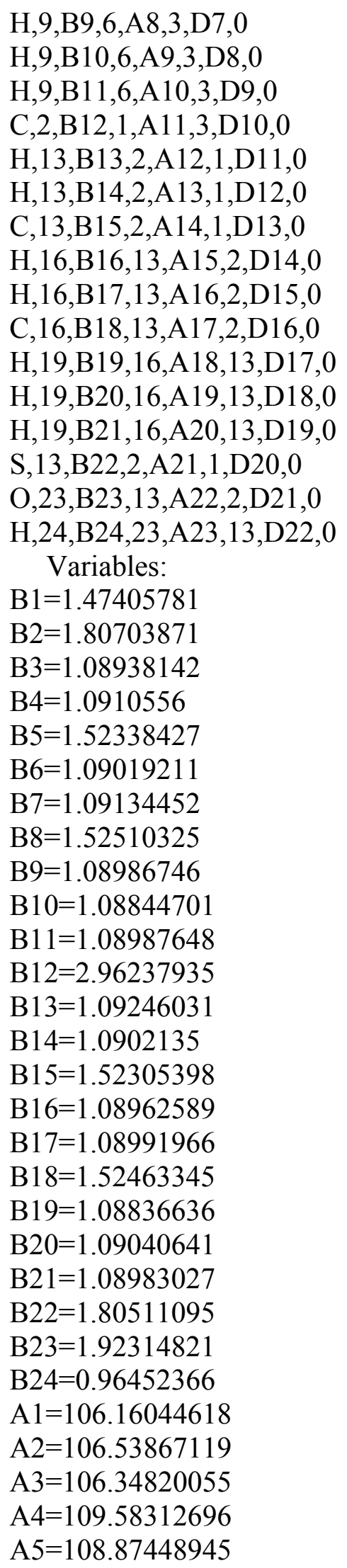




$$
\begin{aligned}
& \mathrm{A} 6=109.44210636 \\
& \mathrm{~A} 7=111.05702468 \\
& \mathrm{~A} 8=111.00228635 \\
& \text { A9 }=110.9059125 \\
& \mathrm{~A} 10=110.78272182 \\
& \mathrm{~A} 11=86.2563834 \\
& \text { A12 }=68.10558002 \\
& \mathrm{~A} 13=145.16698743 \\
& \text { A14 }=101.63966377 \\
& \text { A } 15=109.17126159 \\
& \text { A16 }=109.35023673 \\
& \text { A17 }=110.53379819 \\
& \mathrm{~A} 18=110.81905421 \\
& \text { A19 }=111.105803 \\
& \text { A } 20=110.93235342 \\
& \text { A21 }=48.93048489 \\
& \text { A22 }=106.10016404 \\
& \text { A23 }=102.49872244 \\
& \mathrm{D} 1=168.5315162 \\
& \text { D2 }=50.92085344 \\
& \text { D3 }=-69.4755304 \\
& \text { D4 }=55.95318611 \\
& \text { D5 }=-60.94609525 \\
& \text { D6 }=177.45380304 \\
& \mathrm{D} 7=59.73376547 \\
& \text { D8 }=179.90574782 \\
& \text { D9 }=-60.17563204 \\
& \text { D10 }=130.89236206 \\
& \text { D1 } 1=20.41018267 \\
& \text { D12 }=-70.40306216 \\
& \text { D13 }=129.07596819 \\
& \text { D14 }=-7.65591954 \\
& \text { D15 }=108.22504825 \\
& \text { D16 }=-129.63310713 \\
& \text { D17 }=-179.55401339 \\
& \text { D18 }=-59.50598998 \\
& \text { D19 }=60.48262649 \\
& \text { D20 }=-119.37146388 \\
& \text { D21 }=-78.80153268 \\
& \text { D22 }=-105.50472791
\end{aligned}
$$

\section{$\underline{\text { DPTS••OH (RC8) }}$}

Final structure in terms of initial Z-matrix:

$\mathrm{O}$

$\mathrm{S}, 1, \mathrm{~B} 1$ 


\section{$\mathrm{C}, 2, \mathrm{~B} 2,1, \mathrm{~A} 1$}

$\mathrm{H}, 3, \mathrm{~B} 3,2, \mathrm{~A} 2,1, \mathrm{D} 1,0$

$\mathrm{H}, 3, \mathrm{~B} 4,2, \mathrm{~A} 3,1, \mathrm{D} 2,0$

C,3,B5,2,A4,1,D3,0

$\mathrm{H}, 6, \mathrm{~B} 6,3, \mathrm{~A} 5,2, \mathrm{D} 4,0$

H,6,B7,3,A6,2,D5,0

$\mathrm{C}, 6, \mathrm{~B} 8,3, \mathrm{~A} 7,2, \mathrm{D} 6,0$

$\mathrm{H}, 9, \mathrm{~B} 9,6, \mathrm{~A} 8,3, \mathrm{D} 7,0$

H,9,B10,6,A9,3,D8, 0

H,9,B1 1,6,A10,3,D9, 0

$\mathrm{C}, 2, \mathrm{~B} 12,1, \mathrm{~A} 11,3, \mathrm{D} 10,0$

H,13,B13,2,A12,1,D11,0

H,13,B14,2,A13,1,D12,0

C,13,B15,2,A14,1,D13,0

H,16,B16,13,A15,2,D14,0

H,16,B17,13,A16,2,D15,0

C,16,B18,13,A17,2,D16,0

H,19,B19,16,A18,13,D17,0

H,19,B20,16,A 19, 13,D18,0

H,19,B21,16,A20,13,D19,0

S, 13,B22,2,A21,1,D20,0

$\mathrm{O}, 23, \mathrm{~B} 23,13, \mathrm{~A} 22,2, \mathrm{D} 21,0$

$\mathrm{H}, 24, \mathrm{~B} 24,23, \mathrm{~A} 23,13$,D22, 0

Variables:

$\mathrm{B} 1=1.47129651$

$\mathrm{B} 2=1.80938817$

$\mathrm{B} 3=1.08861346$

B4 $=1.09057816$

B5 $=1.52322709$

B6 $=1.09016985$

B7 $=1.09145654$

$\mathrm{B} 8=1.52499339$

B9 $=1.08977449$

$\mathrm{B} 10=1.08832232$

B11 $=1.08987979$

B12 $=2.94455278$

$\mathrm{B} 13=1.09232803$

B14=1.09005099

B15 $=1.52185596$

B16 $=1.08955079$

B17 $=1.08855318$

$\mathrm{B} 18=1.52481393$

$\mathrm{B} 19=1.08831632$

B20 $=1.09056904$

B21 $=1.09006961$

B22 $=1.80982876$ 


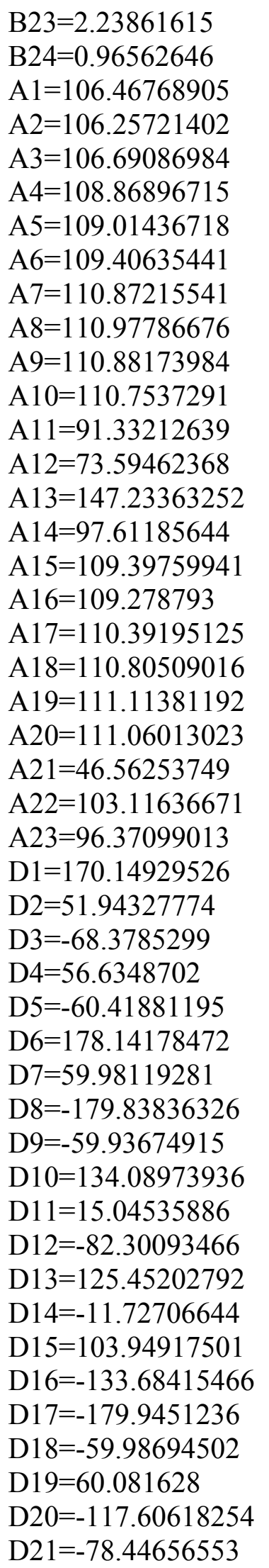


D22=-113.07601991

\section{$\underline{\text { DPTS••OH (PC8) }}$}

Final structure in terms of initial Z-matrix:

$\mathrm{O}$

$\mathrm{S}, 1, \mathrm{~B} 1$

$\mathrm{C}, 2, \mathrm{~B} 2,1, \mathrm{~A} 1$

$\mathrm{H}, 3, \mathrm{~B} 3,2, \mathrm{~A} 2,1, \mathrm{D} 1,0$

$\mathrm{H}, 3, \mathrm{~B} 4,2, \mathrm{~A} 3,1, \mathrm{D} 2,0$

$\mathrm{C}, 3, \mathrm{~B} 5,2, \mathrm{~A} 4,1, \mathrm{D} 3,0$

$\mathrm{H}, 6, \mathrm{~B} 6,3, \mathrm{~A} 5,2, \mathrm{D} 4,0$

$\mathrm{H}, 6, \mathrm{~B} 7,3, \mathrm{~A} 6,2, \mathrm{D} 5,0$

$\mathrm{C}, 6, \mathrm{~B} 8,3, \mathrm{~A} 7,2, \mathrm{D} 6,0$

$\mathrm{H}, 9, \mathrm{~B} 9,6, \mathrm{~A} 8,3, \mathrm{D} 7,0$

$\mathrm{H}, 9, \mathrm{~B} 10,6, \mathrm{~A} 9,3, \mathrm{D} 8,0$

$\mathrm{H}, 9, \mathrm{~B} 11,6, \mathrm{~A} 10,3, \mathrm{D} 9,0$

$\mathrm{C}, 2, \mathrm{~B} 12,1, \mathrm{~A} 11,3, \mathrm{D} 10,0$

$\mathrm{H}, 13, \mathrm{~B} 13,2, \mathrm{~A} 12,1, \mathrm{D} 11,0$

$\mathrm{H}, 13, \mathrm{~B} 14,2, \mathrm{~A} 13,1, \mathrm{D} 12,0$

C,13,B15,2,A14,1,D13,0

$\mathrm{H}, 16, \mathrm{~B} 16,13, \mathrm{~A} 15,2, \mathrm{D} 14,0$

$\mathrm{H}, 16, \mathrm{~B} 17,13$,A 16,2,D15,0

$\mathrm{C}, 16, \mathrm{~B} 18,13, \mathrm{~A} 17,2, \mathrm{D} 16,0$

$\mathrm{H}, 19, \mathrm{~B} 19,16, \mathrm{~A} 18,13, \mathrm{D} 17,0$

H,19,B20,16,A19,13,D18,0

H,19,B21,16,A20,13,D19,0

$\mathrm{S}, 13, \mathrm{~B} 22,2, \mathrm{~A} 21,1, \mathrm{D} 20,0$

$\mathrm{O}, 23, \mathrm{~B} 23,13, \mathrm{~A} 22,2, \mathrm{D} 21,0$

$\mathrm{H}, 24, \mathrm{~B} 24,23, \mathrm{~A} 23,13, \mathrm{D} 22,0$

Variables:

$\mathrm{B} 1=1.49460724$

$\mathrm{B} 2=1.80042937$

$\mathrm{B} 3=1.09155967$

B4 $=1.09181609$

$\mathrm{B} 5=1.52373188$

B6 $=1.09022599$

B7 $=1.09129537$

$\mathrm{B} 8=1.5246474$

$\mathrm{B} 9=1.09006979$

$\mathrm{B} 10=1.08857657$

$\mathrm{B} 11=1.08988677$

$\mathrm{B} 12=3.64169708$

$\mathrm{B} 13=1.09026883$

$\mathrm{B} 14=1.0938556$

$\mathrm{B} 15=1.52072027$ 


$$
\begin{aligned}
& \mathrm{B} 16=1.09218594 \\
& \mathrm{~B} 17=1.09152652 \\
& \mathrm{~B} 18=1.52534819 \\
& \mathrm{~B} 19=1.08872064 \\
& \mathrm{~B} 20=1.09041607 \\
& \mathrm{~B} 21=1.08937655 \\
& \mathrm{~B} 22=1.79948601 \\
& \mathrm{~B} 23=1.66334337 \\
& \mathrm{~B} 24=0.96163072 \\
& \mathrm{~A} 1=105.8189472 \\
& \mathrm{~A} 2=105.43395741 \\
& \mathrm{~A} 3=107.31198096 \\
& \mathrm{~A} 4=112.06567004 \\
& \mathrm{~A} 5=108.552289 \\
& \mathrm{~A} 6=109.38433264 \\
& \mathrm{~A} 7=111.29082442 \\
& \mathrm{~A} 8=111.00837667 \\
& \mathrm{~A} 9=111.00549838 \\
& \mathrm{~A} 10=110.79517961 \\
& \mathrm{~A} 11=67.5491668 \\
& \mathrm{~A} 12=44.18802822 \\
& \mathrm{~A} 13=151.54194913 \\
& \mathrm{~A} 14=83.00909197 \\
& \mathrm{~A} 15=108.73764391 \\
& \mathrm{~A} 16=109.38414125 \\
& \mathrm{~A} 17=111.19731373 \\
& \mathrm{~A} 18=111.02484891 \\
& \mathrm{~A} 19=110.83589041 \\
& \mathrm{~A} 20=110.25367198 \\
& \mathrm{~A} 21=92.71499605 \\
& \mathrm{~A} 22=101.38295043 \\
& \mathrm{~A} 23=108.41539766 \\
& \mathrm{D} 1=176.15520483 \\
& \mathrm{D} 2=61.69710132 \\
& \mathrm{D} 3=-61.16655572 \\
& \mathrm{D} 4=55.69610366 \\
& \mathrm{D} 5=-60.7225419 \\
& \mathrm{D} 6=177.28018717 \\
& \mathrm{D} 7=59.98610372 \\
& \mathrm{D} 8=-179.84367284 \\
& \mathrm{D} 9=-59.76405674 \\
& \mathrm{D} 10=74.1215016 \\
& \mathrm{D} 11=-43.40910263 \\
& \mathrm{D} 12=-36.2016558 \\
& \mathrm{D} 13=85.5952527 \\
& \mathrm{D} 14=29.20734681
\end{aligned}
$$



D15 $=146.21471364$
D16 $=-91.05412172$
$\mathrm{D} 17=179.2458888$
D18 $=-60.73435269$
D19 $=59.26611538$
D20 $=-159.87669349$
D21 $=-25.37539906$
D22 $=-88.71476788$

\section{$\underline{\text { DPTS••OH (TS9) }}$}

Final structure in terms of initial Z-matrix:

$\mathrm{O}$

$\mathrm{S}, 1, \mathrm{~B} 1$

$\mathrm{C}, 2, \mathrm{~B} 2,1, \mathrm{~A} 1$

$\mathrm{H}, 3, \mathrm{~B} 3,2, \mathrm{~A} 2,1, \mathrm{D} 1,0$

$\mathrm{H}, 3, \mathrm{~B} 4,2, \mathrm{~A} 3,1, \mathrm{D} 2,0$

$\mathrm{C}, 3, \mathrm{~B} 5,2, \mathrm{~A} 4,1, \mathrm{D} 3,0$

$\mathrm{H}, 6, \mathrm{~B} 6,3, \mathrm{~A} 5,2, \mathrm{D} 4,0$

$\mathrm{H}, 6, \mathrm{~B} 7,3, \mathrm{~A} 6,2, \mathrm{D} 5,0$

$\mathrm{C}, 6, \mathrm{~B} 8,3, \mathrm{~A} 7,2, \mathrm{D} 6,0$

$\mathrm{H}, 9, \mathrm{~B} 9,6, \mathrm{~A} 8,3, \mathrm{D} 7,0$

H,9,B10,6,A9,3,D8,0

H,9,B11,6,A10,3,D9,0

C,2,B12,1,A11,3,D10,0

H,13,B13,2,A12,1,D11,0

H,13,B14,2,A13, 1,D12,0

C,13,B15,2,A14,1,D13,0

H,16,B16,13,A15,2,D14,0

H,16,B17,13,A16,2,D15,0

C,16,B18,13,A17,2,D16,0

H,19,B19,16,A18,13,D17,0

H,19,B20,16,A19,13,D18,0

H,19,B21,16,A20,13,D19,0

S, 13,B22,2,A21,1,D20,0

O,2,B23, 1,A22,3,D21,0

H,24,B24,2,A23, 1,D22,0

Variables:

$\mathrm{B} 1=1.47561143$

$\mathrm{B} 2=1.79895771$

$\mathrm{B} 3=1.08736434$

$\mathrm{B} 4=1.08892693$

$\mathrm{B} 5=1.52250215$

B6 $=1.08985802$

B7 $=1.08818524$ 


$$
\begin{aligned}
& B 8=1.52566966 \\
& B 9=1.08979554 \\
& B 10=1.08821805 \\
& B 11=1.0898904 \\
& B 12=2.92846746 \\
& B 13=1.08948548 \\
& B 14=1.09065998 \\
& B 15=1.52132037 \\
& B 16=1.09155914 \\
& B 17=1.09030868 \\
& B 18=1.52478533 \\
& B 19=1.08834809 \\
& B 20=1.09025836 \\
& B 21=1.08965634 \\
& B 22=1.81339263 \\
& B 23=1.92236494 \\
& B 24=0.96429059 \\
& A 1=103.63806294 \\
& A 2=107.08387212 \\
& A 3=104.07186415 \\
& A 4=110.85957168 \\
& A 5=108.96658359 \\
& A 6=108.99676014 \\
& A 7=110.30482651 \\
& A 8=110.95627848 \\
& A 9=110.70437552 \\
& A 10=111.07011151 \\
& A 11=78.83473358 \\
& A 12=77.0368479 \\
& A 13=148.55944845 \\
& A 14=94.74488075 \\
& A 15=108.92212092 \\
& A 16=109.29987547 \\
& A 17=111.04774538 \\
& A 18=111.08595475 \\
& A 19=110.91605746 \\
& A 20=110.7389553 \\
& A 21=46.89789364 \\
& A 22=142.99298564 \\
& A 23=104.86100491 \\
& D 1=167.69911189 \\
& D 2=50.72175731 \\
& D 3=-69.60243064 \\
& D 4=55.37535939 \\
& D 5=-62.13522647 \\
& D 6=176.38938331
\end{aligned}
$$




$$
\begin{aligned}
& D 7=60.24058119 \\
& D 8=-179.89341414 \\
& D 9=-59.9310844 \\
& D 10=126.02290205 \\
& D 11=-14.63867958 \\
& D 12=-118.03103901 \\
& D 13=96.02814744 \\
& D 14=-16.60775112 \\
& D 15=100.17642358 \\
& D 16=-137.47772392 \\
& D 17=179.84092124 \\
& D 18=-60.07358187 \\
& D 19=59.80446568 \\
& D 20=-144.70119175 \\
& D 21=-101.8137998 \\
& D 22=-76.55446337
\end{aligned}
$$

\section{DPTS••OH (RC9)}

Final structure in terms of initial Z-matrix:

$\mathrm{O}$

$\mathrm{S}, 1, \mathrm{~B} 1$

$\mathrm{C}, 2, \mathrm{~B} 2,1, \mathrm{~A} 1$

$\mathrm{H}, 3, \mathrm{~B} 3,2, \mathrm{~A} 2,1, \mathrm{D} 1,0$

$\mathrm{H}, 3, \mathrm{~B} 4,2, \mathrm{~A} 3,1, \mathrm{D} 2,0$

$\mathrm{C}, 3, \mathrm{~B} 5,2, \mathrm{~A} 4,1, \mathrm{D} 3,0$

$\mathrm{H}, 6, \mathrm{~B} 6,3, \mathrm{~A} 5,2, \mathrm{D} 4,0$

H,6,B7,3,A6,2,D5,0

C,6,B8,3,A7,2,D6,0

$\mathrm{H}, 9, \mathrm{~B} 9,6, \mathrm{~A} 8,3, \mathrm{D} 7,0$

H,9,B10,6,A9,3,D8,0

H,9,B11,6,A10,3,D9,0

C,1,B12,2,A11,3,D10,0

$\mathrm{H}, 13, \mathrm{~B} 13,1, \mathrm{~A} 12,2, \mathrm{D} 11,0$

$\mathrm{H}, 13, \mathrm{~B} 14,1, \mathrm{~A} 13,2, \mathrm{D} 12,0$

C,13,B15,1,A14,2,D13,0

$\mathrm{H}, 16, \mathrm{~B} 16,13, \mathrm{~A} 15,1, \mathrm{D} 14,0$

H,16,B17,13,A16,1,D15,0

C,16,B18,13,A17,1,D16,0

H,19,B19,16,A18,13,D17,0

H,19,B20,16,A19,13,D18,0

H,19,B21,16,A20,13,D19,0

S, 13,B22,1,A21,2,D20,0

O,2,B23,1,A22,3,D21,0

H,24,B24,2,A23, 1,D22,0

Variables: 


$$
\begin{aligned}
& B 1=1.48843671 \\
& B 2=1.80048833 \\
& B 3=1.08557028 \\
& B 4=1.08958523 \\
& B 5=1.52251195 \\
& B 6=1.0899476 \\
& B 7=1.08968944 \\
& B 8=1.52535398 \\
& B 9=1.08967158 \\
& B 10=1.08824058 \\
& B 11=1.08989949 \\
& B 12=2.86032029 \\
& B 13=1.08895075 \\
& B 14=1.09127619 \\
& B 15=1.52142754 \\
& B 16=1.08894091 \\
& B 17=1.09082839 \\
& B 18=1.52456475 \\
& B 19=1.08839913 \\
& B 20=1.09058905 \\
& B 21=1.08991038 \\
& B 22=1.81201547 \\
& B 23=1.89964234 \\
& B 24=0.96393579 \\
& A 1=102.71903512 \\
& A 2=107.16316528 \\
& A 3=104.36843723 \\
& A 4=110.07478491 \\
& A 5=109.12860964 \\
& A 6=108.71928308 \\
& A 7=110.60243198 \\
& A 8=110.92328715 \\
& A 9=110.78856139 \\
& A 10=110.96744236 \\
& A 11=80.35360428 \\
& A 12=55.1161322 \\
& A 13=158.2671269 \\
& A 14=89.00828529 \\
& A 15=108.50470919 \\
& A 16=109.12613521 \\
& A 17=110.98234788 \\
& A 18=111.0490017 \\
& A 19=111.13657984 \\
& A 20=110.64158968 \\
& A 21=72.81826053 \\
& A 22=159.88357837
\end{aligned}
$$



A23 $=106.2026354$
D1 $=161.38290361$
D2 $=43.92828576$
D3 $=-76.1954512$
D4 $=56.4054158$
D5 $=-60.94336911$
D6 $=177.82082397$
$\mathrm{D} 7=59.98214385$
D8 $=179.94918927$
D9=-60.10248709
D10 $=-104.83744055$
D1 $1=122.78893803$
D12 $=80.17201964$
D13 $=-120.59897143$
D14 $=11.14852073$
D15 $=128.02270067$
D16 $=-110.03344391$
D17 $=-179.38138712$
D18 $=-59.19944226$
D19 $=60.76599652$
D20 $=-5.11725204$
D21 $=-116.50163761$
D22 $=-55.45393982$

\section{DPTS••OH (PC9)}

\section{$\mathrm{CH}_{3} \underline{\mathrm{CH}}_{2} \underline{\mathrm{CH}} \underline{2} \underline{\mathrm{S}(=\mathrm{O}) \mathrm{OH}}$}

Final structure in terms of initial Z-matrix:

$\mathrm{O}$

$\mathrm{S}, 1, \mathrm{~B} 1$

$\mathrm{C}, 2, \mathrm{~B} 2,1, \mathrm{~A} 1$

H,3,B3, 2,A2, 1,D1,0

$\mathrm{H}, 3, \mathrm{~B} 4,2, \mathrm{~A} 3,1, \mathrm{D} 2,0$

$\mathrm{C}, 3, \mathrm{~B} 5,2, \mathrm{~A} 4,1, \mathrm{D} 3,0$

$\mathrm{H}, 6, \mathrm{~B} 6,3, \mathrm{~A} 5,2, \mathrm{D} 4,0$

H,6,B7,3,A6,2,D5,0

$\mathrm{C}, 6, \mathrm{~B} 8,3, \mathrm{~A} 7,2, \mathrm{D} 6,0$

H,9,B9,6,A8,3,D7,0

$\mathrm{H}, 9, \mathrm{~B} 10,6, \mathrm{~A} 9,3, \mathrm{D} 8,0$

H,9,B11,6,A10,3,D9,0

O,2,B12,1,A11,3,D10,0

H,13,B13,2,A12,1,D11,0

Variables:

$\mathrm{B} 1=1.45944056$

$\mathrm{B} 2=1.80176835$ 


$$
\begin{aligned}
& B 3=1.08992448 \\
& \mathrm{~B} 4=1.09131976 \\
& \text { B5 }=1.5210965 \\
& \text { B6 }=1.08919837 \\
& \text { B7 }=1.08853941 \\
& \mathrm{~B} 8=1.52534862 \\
& \text { B9 }=1.09009561 \\
& \mathrm{~B} 10=1.08843574 \\
& \text { B11 }=1.09003327 \\
& \mathrm{~B} 12=1.64758807 \\
& \text { B13 }=0.9633496 \\
& \mathrm{~A} 1=106.43053625 \\
& \mathrm{~A} 2=106.59291517 \\
& \text { A3 }=103.99563944 \\
& \text { A4 }=112.90642534 \\
& \text { A5 }=108.56548166 \\
& \text { A6 }=109.17935495 \\
& \text { A7 }=110.94530697 \\
& \text { A } 8=111.16278397 \\
& \text { A9 }=110.8018339 \\
& \text { A } 10=110.99547692 \\
& \text { A11 }=108.68984835 \\
& \text { A12 }=108.26260294 \\
& \text { D1 }=-171.02411825 \\
& \text { D2 }=74.19471163 \\
& \text { D3 }=-46.61038692 \\
& \mathrm{D} 4=52.12792218 \\
& \text { D5 }=-64.43565903 \\
& \text { D6 }=173.76383598 \\
& \text { D7 }=59.90336405 \\
& \text { D8 }=179.91885583 \\
& \text { D9 }=-60.20242556 \\
& \text { D10 }=-98.52255537 \\
& \text { D11 }=-81.53302174
\end{aligned}
$$

\section{$\underline{\mathrm{CH}}_{3} \underline{\mathrm{CH}_{2}} \underline{\mathrm{CH}_{2}} \underline{\mathrm{S}}$}

Final structure in terms of initial Z-matrix:
$\mathrm{C}$
$\mathrm{C}, 1, \mathrm{~B} 1$
$\mathrm{H}, 1, \mathrm{~B} 2,2, \mathrm{~A} 1$
$\mathrm{H}, 1, \mathrm{~B} 3,2, \mathrm{~A} 2,3, \mathrm{D} 1,0$
$\mathrm{H}, 1, \mathrm{~B} 4,2, \mathrm{~A} 3,4, \mathrm{D} 2,0$
$\mathrm{C}, 2, \mathrm{~B} 5,1, \mathrm{~A} 4,5, \mathrm{D} 3,0$
$\mathrm{H}, 2, \mathrm{~B} 6,1, \mathrm{~A} 5,6, \mathrm{D} 4,0$
$\mathrm{H}, 2, \mathrm{~B} 7,1, \mathrm{~A} 6,6, \mathrm{D} 5,0$ 


$$
\begin{aligned}
& \mathrm{H}, 6, \mathrm{~B} 8,2, \mathrm{~A} 7,1, \mathrm{D} 6,0 \\
& \mathrm{H}, 6, \mathrm{~B} 9,2, \mathrm{~A} 8,1, \mathrm{D} 7,0 \\
& \mathrm{~S}, 6, \mathrm{~B} 10,2, \mathrm{~A} 9,1, \mathrm{D} 8,0 \\
& \text { Variables: } \\
& \mathrm{B} 1=1.52476624 \\
& \mathrm{~B} 2=1.08855018 \\
& \mathrm{~B} 3=1.09041224 \\
& \mathrm{~B} 4=1.09041224 \\
& \mathrm{~B} 5=1.52230791 \\
& \mathrm{~B} 6=1.09035869 \\
& \mathrm{~B} 7=1.09035869 \\
& \mathrm{~B} 8=1.09379065 \\
& \mathrm{~B} 9=1.09379065 \\
& \mathrm{~B} 10=1.79242847 \\
& \mathrm{~A} 1=111.22585284 \\
& \mathrm{~A} 2=110.93643436 \\
& \mathrm{~A} 3=110.93643436 \\
& \mathrm{~A} 4=111.52357608 \\
& \mathrm{~A} 5=110.37196483 \\
& \mathrm{~A} 6=110.37196487 \\
& \mathrm{~A} 7=111.03062989 \\
& \mathrm{~A} 8=111.03062987 \\
& \text { A9 }=115.43910985 \\
& \mathrm{D} 1=120.13149454 \\
& \mathrm{D} 2=119.73701094 \\
& \mathrm{D} 3=59.86850549 \\
& \mathrm{D} 4=121.19500562 \\
& \text { D5 }=-121.19500565 \\
& \text { D6 }=-58.49343348 \\
& \mathrm{D} 7=58.49343344 \\
& \mathrm{D} 8=180 .
\end{aligned}
$$

\section{$\underline{\text { DPTS••OH (TS10) }}$}

Final structure in terms of initial Z-matrix:

$\mathrm{O}$

$\mathrm{S}, 1, \mathrm{~B} 1$

$\mathrm{C}, 2, \mathrm{~B} 2,1, \mathrm{~A} 1$

$\mathrm{H}, 3, \mathrm{~B} 3,2, \mathrm{~A} 2,1, \mathrm{D} 1,0$

$\mathrm{H}, 3, \mathrm{~B} 4,2, \mathrm{~A} 3,1, \mathrm{D} 2,0$

C,3,B5,2,A4,1,D3,0

H,6,B6,3,A5,2,D4,0

$\mathrm{H}, 6, \mathrm{~B} 7,3, \mathrm{~A} 6,2, \mathrm{D} 5,0$

$\mathrm{C}, 6, \mathrm{~B} 8,3, \mathrm{~A} 7,2, \mathrm{D} 6,0$

H,9,B9,6,A8,3,D7,0

H,9,B10,6,A9,3,D8,0 


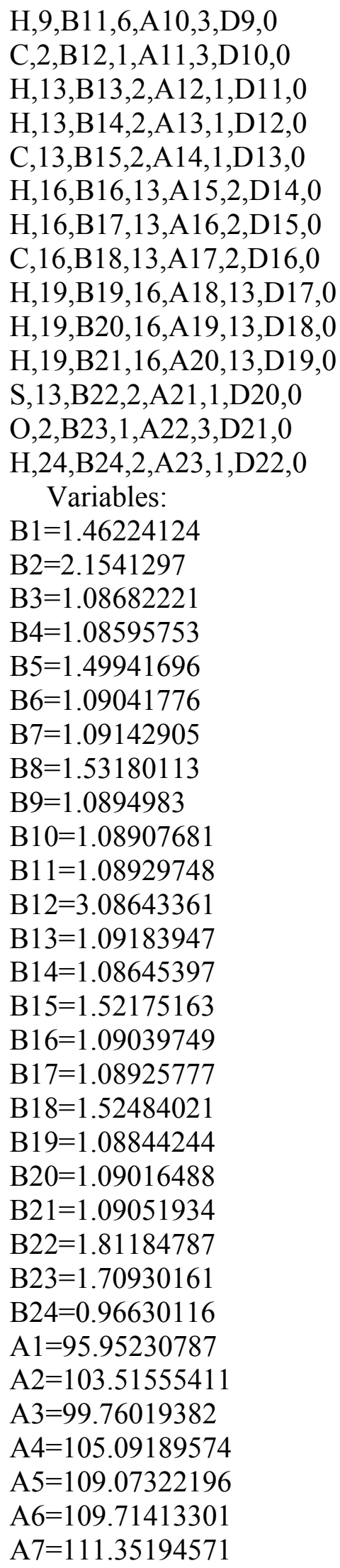




$$
\begin{aligned}
& \mathrm{A} 8=110.96419165 \\
& \text { A9 }=110.71423884 \\
& \text { A } 10=110.74356394 \\
& \text { A11 }=99.32841366 \\
& \text { A12 }=144.23111345 \\
& \mathrm{~A} 13=84.71930589 \\
& \text { A14 }=93.79374643 \\
& \text { A15 }=109.14857745 \\
& \text { A16 }=109.22528193 \\
& \text { A17 }=110.79898143 \\
& \mathrm{~A} 18=111.04562291 \\
& \text { A19 }=110.86749089 \\
& \text { A20 }=111.06172088 \\
& \mathrm{~A} 21=41.51408022 \\
& \text { A22 }=100.40794025 \\
& \text { A23 }=104.41918046 \\
& \mathrm{D} 1=169.79825511 \\
& \text { D2 }=52.32627295 \\
& \text { D3 }=-67.94719638 \\
& \text { D4 }=54.46646162 \\
& \text { D5 }=-62.94418886 \\
& \text { D6 }=175.69132234 \\
& \text { D7 }=59.81046452 \\
& \text { D8 }=179.95419519 \\
& \text { D9 }=-60.10049299 \\
& \text { D10 }=123.7452869 \\
& \text { D11 }=-130.46374171 \\
& \text { D12 }=115.45480259 \\
& \mathrm{D} 13=4.06629465 \\
& \text { D14=-93.75765581 } \\
& \text { D15 }=22.44268572 \\
& \text { D16 }=144.34588106 \\
& \mathrm{D} 17=179.75565639 \\
& \text { D18 }=-60.21578488 \\
& \mathrm{D} 19=59.6250845 \\
& \text { D20 }=-118.86596928 \\
& \text { D21 }=-169.28548945 \\
& \text { D22 }=21.56184888
\end{aligned}
$$

\section{$\underline{\text { DPTS••OH (RC10) }}$}

Final structure in terms of initial Z-matrix:

$\mathrm{O}$

$\mathrm{S}, 1, \mathrm{~B} 1$

$\mathrm{C}, 2, \mathrm{~B} 2,1, \mathrm{~A} 1$

$\mathrm{H}, 3, \mathrm{~B} 3,2, \mathrm{~A} 2,1, \mathrm{D} 1,0$ 


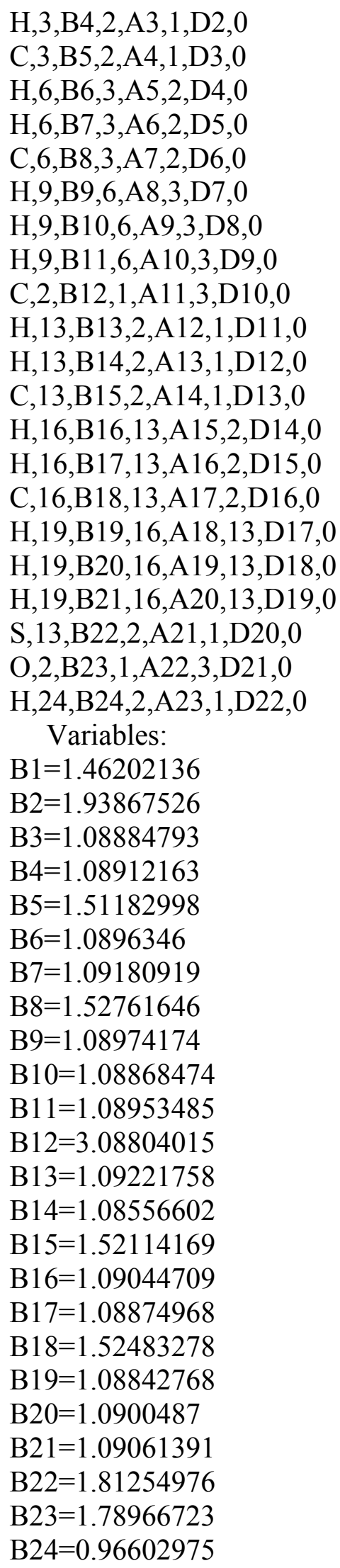



$\mathrm{A} 1=99.73364751$
$\mathrm{A} 2=105.85102365$
$\mathrm{A} 3=104.26440746$
A4 $=107.95773697$
A5 $=108.90954075$
A6 $=109.51875916$
$\mathrm{A} 7=111.21914156$
A $8=111.0389868$
A9 $=110.82078711$
A10 $=110.80374193$
A $11=100.9487775$
$\mathrm{A} 12=143.37838605$
$\mathrm{A} 13=86.41924184$
A14 $=92.94979229$
A15 $=109.20312434$
A16 $=109.06713257$
A $17=110.64411982$
A18 $=110.99375979$
A19 $=110.77533996$
A20 $=111.13971187$
A21 $=40.92938844$
$\mathrm{A} 22=99.15507532$
$\mathrm{A} 23=102.80011811$
D1 $=173.03121407$
D2 $=55.48067164$
D3 $=-65.20102942$
D4 $=56.9406119$
D5 $=-60.10562021$
D6 $=178.4789166$
D7 $=59.79468106$
D8 $=179.92805316$
D9 $=-60.15407963$
D10 $=128.75636348$
D11 $=-126.79656405$
D12 $=116.59754732$
D13 $=4.97368905$
D14=-92.83174761
D15 $=23.70417555$
D16 $=145.30918346$
D17 $=179.51939897$
D18 $=-60.541976$
D19 $=59.37996468$
D20 $=-119.39517253$
D21 $=-166.06549033$
D22 $=34.98959712$ 


\section{$\underline{\text { DPTS} \bullet \circ O H ~(P C 10) ~}$}

Final structure in terms of initial Z-matrix:

$\mathrm{O}$

$\mathrm{S}, 1, \mathrm{~B} 1$

$\mathrm{C}, 2, \mathrm{~B} 2,1, \mathrm{~A} 1$

H,3,B3, 2,A2, 1,D1,0

H,3,B4, 2,A3, 1,D2,0

C,3,B5,2,A4, 1,D3,0

$\mathrm{H}, 6, \mathrm{~B} 6,3, \mathrm{~A} 5,2, \mathrm{D} 4,0$

$\mathrm{H}, 6, \mathrm{~B} 7,3, \mathrm{~A} 6,2, \mathrm{D} 5,0$

$\mathrm{C}, 6, \mathrm{~B} 8,3, \mathrm{~A} 7,2, \mathrm{D} 6,0$

$\mathrm{H}, 9, \mathrm{~B} 9,6, \mathrm{~A} 8,3, \mathrm{D} 7,0$

$\mathrm{H}, 9, \mathrm{~B} 10,6, \mathrm{~A} 9,3, \mathrm{D} 8,0$

H,9,B11,6,A10,3,D9,0

C,2,B12,1,A11,3,D10,0

$\mathrm{H}, 13, \mathrm{~B} 13,2, \mathrm{~A} 12,1, \mathrm{D} 11,0$

H,13,B14,2,A13,1,D12,0

$\mathrm{C}, 13, \mathrm{~B} 15,2, \mathrm{~A} 14,1, \mathrm{D} 13,0$

$\mathrm{H}, 16, \mathrm{~B} 16,13, \mathrm{~A} 15,2, \mathrm{D} 14,0$

$\mathrm{H}, 16, \mathrm{~B} 17,13, \mathrm{~A} 16,2, \mathrm{D} 15,0$

C,16,B18,13,A17,2,D16,0

H,19,B19,16,A18,13,D17,0

H,19,B20,16,A19,13,D18,0

H,19,B21,16,A20,13,D19,0

S, 13,B22,2,A21,1,D20,0

$\mathrm{O}, 2, \mathrm{~B} 23,1, \mathrm{~A} 22,3, \mathrm{D} 21,0$

H,24,B24,2,A23, 1,D22,0

Variables:

B1 $=1.45393202$

$\mathrm{B} 2=3.24575247$

$\mathrm{B} 3=1.08002536$

B4 $=1.08044604$

$\mathrm{B} 5=1.48823751$

$\mathrm{B} 6=1.09211853$

B7 $=1.09091969$

$\mathrm{B} 8=1.53704523$

B9 $=1.08929952$

$\mathrm{B} 10=1.08946812$

$\mathrm{B} 11=1.08946753$

$\mathrm{B} 12=3.02846811$

$\mathrm{B} 13=1.09117682$

$\mathrm{B} 14=1.088136$

$\mathrm{B} 15=1.51966227$

$\mathrm{B} 16=1.09079686$

B17 $=1.08938384$ 


$$
\begin{aligned}
& \mathrm{B} 18=1.52492096 \\
& \mathrm{~B} 19=1.08847846 \\
& \mathrm{~B} 20=1.08995279 \\
& \text { B21 }=1.09045039 \\
& \mathrm{~B} 22=1.81457387 \\
& \mathrm{~B} 23=1.6165753 \\
& \mathrm{~B} 24=0.96884957 \\
& \mathrm{~A} 1=92.0662177 \\
& \mathrm{~A} 2=82.54709072 \\
& \mathrm{~A} 3=118.68457371 \\
& \mathrm{~A} 4=84.26593294 \\
& \mathrm{~A} 5=110.11675478 \\
& \text { A6 }=110.03804528 \\
& \text { A } 7=112.0757699 \\
& \mathrm{~A} 8=110.69874189 \\
& \mathrm{~A} 9=110.83145725 \\
& \text { A } 10=110.5989864 \\
& \mathrm{~A} 11=69.52259388 \\
& \mathrm{~A} 12=143.66632056 \\
& \text { A13 }=75.74679709 \\
& \text { A14 }=100.14826709 \\
& \text { A15 }=109.34622004 \\
& \text { A16 }=108.55073113 \\
& \text { A } 17=110.86480225 \\
& \text { A18 }=111.06675996 \\
& \text { A19 }=110.74721308 \\
& \text { A20 }=111.09090561 \\
& \text { A21 }=44.42922816 \\
& \text { A22 }=108.77737327 \\
& \text { A23 }=110.83064922 \\
& \text { D1 }=-163.51540964 \\
& \text { D2 }=79.65197713 \\
& \text { D3 }=-41.54041579 \\
& \text { D4 }=71.62727549 \\
& \text { D5 }=-45.19848667 \\
& \text { D6 }=-166.86861929 \\
& \text { D7 }=59.88664705 \\
& \text { D8 }=-179.79790091 \\
& \text { D9 }=-59.64574471 \\
& \text { D10 }=85.12503889 \\
& \text { D1 } 1=135.048317 \\
& \text { D12 }=34.1356208 \\
& \text { D13 }=-75.09352497 \\
& \text { D14 }=-97.85889096 \\
& \text { D15 }=18.82575329 \\
& \text { D16 }=139.89626147
\end{aligned}
$$



$\mathrm{D} 17=-179.66919217$
D18 $=-59.75183661$
D19 $=60.18909197$
D20 $=169.78912479$
D21 $=-165.53745244$
D22 $=-57.68954283$

\section{$\underline{\mathrm{CH}}_{3} \underline{\mathrm{CH}}_{2} \underline{\mathrm{CH}_{2}}$}

Final structure in terms of initial Z-matrix:

C

$\mathrm{H}, 1, \mathrm{~B} 1$

$\mathrm{H}, 1, \mathrm{~B} 2,2, \mathrm{~A} 1$

H, 1,B3, 2,A2,3,D1,0

$\mathrm{C}, 1, \mathrm{~B} 4,2, \mathrm{~A} 3,4, \mathrm{D} 2,0$

$\mathrm{H}, 5, \mathrm{~B} 5,1, \mathrm{~A} 4,2, \mathrm{D} 3,0$

$\mathrm{H}, 5, \mathrm{~B} 6,1, \mathrm{~A} 5,2, \mathrm{D} 4,0$

$\mathrm{C}, 5, \mathrm{~B} 7,1, \mathrm{~A} 6,2, \mathrm{D} 5,0$

$\mathrm{H}, 8, \mathrm{~B} 8,5, \mathrm{~A} 7,1, \mathrm{D} 6,0$

$\mathrm{H}, 8, \mathrm{~B} 9,5, \mathrm{~A} 8,1, \mathrm{D} 7,0$

Variables:

$\mathrm{B} 1=1.08968157$

$\mathrm{B} 2=1.08921023$

$\mathrm{B} 3=1.08921686$

$\mathrm{B} 4=1.53832765$

$\mathrm{B} 5=1.09112983$

B6 $=1.09112435$

$\mathrm{B} 7=1.48656397$

$\mathrm{B} 8=1.07966103$

$\mathrm{B} 9=1.07966051$

$\mathrm{A} 1=108.40548077$

$\mathrm{A} 2=108.40816716$

A3 $=110.93992475$

A4 $=108.72513077$

A5 $=108.72307951$

$\mathrm{A} 6=112.29226582$

$\mathrm{A} 7=120.60589431$

A8 $=120.59409779$

D1 $=-116.88033695$

$\mathrm{D} 2=-121.55801725$

D3 $=-58.10082967$

D4 $=58.03156091$

D5 $=179.96434027$

D6 $=83.66735236$

$\mathrm{D} 7=-83.76872432$ 


\section{$\underline{\mathrm{CH}}_{3} \underline{\mathrm{CH}}_{2} \underline{\mathrm{CH}_{2}} \underline{\mathrm{SS}(=\mathrm{O}) \mathrm{OH}}$}

Final structure in terms of initial Z-matrix:

$\mathrm{O}$

$\mathrm{S}, 1, \mathrm{~B} 1$

$\mathrm{C}, 2, \mathrm{~B} 2,1, \mathrm{~A} 1$

H,3,B3, 2,A2, 1,D1,0

H,3,B4, 2,A3, 1,D2,0

C,3,B5,2,A4, 1,D3,0

H,6,B6,3,A5,2,D4,0

H,6,B7,3,A6,2,D5,0

$\mathrm{C}, 6, \mathrm{~B} 8,3, \mathrm{~A} 7,2, \mathrm{D} 6,0$

H,9,B9,6,A8,3,D7,0

H,9,B10,6,A9,3,D8,0

H,9,B11,6,A10,3,D9,0

S,3,B12,2,A11,1,D10,0

$\mathrm{O}, 2, \mathrm{~B} 13,1, \mathrm{~A} 12,13, \mathrm{D} 11,0$

H,14,B14,2,A13,1,D12,0

Variables:

B1 $=1.45103046$

$\mathrm{B} 2=2.97318015$

$\mathrm{B} 3=1.09056722$

B4 $=1.08870267$

$\mathrm{B} 5=1.52134469$

B6 $=1.0901512$

$\mathrm{B} 7=1.091712$

$\mathrm{B} 8=1.52468371$

$\mathrm{B} 9=1.08829766$

$\mathrm{B} 10=1.08966821$

$\mathrm{B} 11=1.0902274$

$\mathrm{B} 12=1.81353063$

$\mathrm{B} 13=1.61249686$

B14 $=0.96987632$

$\mathrm{A} 1=76.188763$

$\mathrm{A} 2=147.78828259$

A $3=77.89222084$

A4 $=94.99478824$

$\mathrm{A} 5=109.29660782$

$\mathrm{A} 6=109.15979758$

$\mathrm{A} 7=111.07699656$

$\mathrm{A} 8=111.06576611$

A9 $=110.79165805$

$\mathrm{A} 10=110.88669688$

A11 $=45.54228415$

A12 $=109.69814537$

$\mathrm{A} 13=110.32362172$ 

D1 $=115.82645387$
D2 $=11.2529105$
D3 $=-99.63075572$
D4 $=-98.83660427$
D5 $=17.79393453$
D6 $=138.83628816$
D7 $=-179.81900583$
$\mathrm{D} 8=-59.75600895$
D9 $=60.11773896$
D10 $=141.09324389$
D1 $1=100.71459953$
D12 $=-54.20872519$ 
Table S7: The calculated tunneling contributions for all the possible abstraction and substitution pathways involved in the DPTS $+\bullet \mathrm{OH}$ reaction in the temperatures between 200 and $300 \mathrm{~K}$ and at 760 Torr pressure.

\begin{tabular}{|c|c|c|c|c|c|c|c|c|c|c|c|c|c|c|c|c|}
\hline $\mathbf{T}$ & $\mathbf{k}_{\mathbf{1}}$ & $\mathbf{k}_{\mathbf{2}}$ & $\mathbf{k}_{\mathbf{3}}$ & $\mathbf{k}_{\mathbf{4}}$ & $\mathbf{2}_{\mathbf{5}}$ & $\mathbf{k}_{\mathbf{6}}$ & $\mathbf{k}_{\mathbf{7}}$ & $\mathbf{k}_{\mathbf{8}}$ & $\mathbf{k}_{\mathbf{9}}$ & $\mathbf{k}_{\mathbf{1 0}}$ & $\mathbf{2}_{\mathbf{1 1}}$ & $\mathbf{k}_{\mathbf{1 2}}$ & $\mathbf{k}_{\mathbf{1 3}}$ & $\mathbf{k}_{\mathbf{1 4}}$ & $\mathbf{k}_{\mathbf{1 5}}$ & $\mathbf{k}_{\mathbf{1 6}}$ \\
\hline 200 & 6 & 12 & 3 & 4 & 4 & 3 & 2 & 2 & 4 & 3 & 5 & 12 & 1.8 & 1.1 & 1.2 & 1.1 \\
\hline 220 & 4 & 8 & 3 & 4 & 4 & 3 & 2 & 2 & 3 & 2 & 4 & 9 & 1.7 & 1.1 & 1.1 & 1.0 \\
\hline 240 & 4 & 6 & 2 & 3 & 3 & 2 & 2 & 2 & 3 & 2 & 3 & 8 & 1.6 & 1.1 & 1.0 & 1.0 \\
\hline 250 & 3 & 5 & 2 & 3 & 3 & 2 & 2 & 2 & 3 & 2 & 3 & 7 & 1.5 & 1.1 & 1.0 & 1.0 \\
\hline 260 & 3 & 4 & 2 & 3 & 3 & 2 & 2 & 2 & 2 & 2 & 2 & 6 & 1.5 & 1.1 & 1.0 & 1.0 \\
\hline 280 & 3 & 4 & 2 & 2 & 2 & 2 & 2 & 2 & 2 & 2 & 2 & 5 & 1.4 & 1.0 & 1.0 & 1.0 \\
\hline 300 & 3 & 3 & 2 & 2 & 2 & 2 & 2 & 2 & 2 & 2 & 2 & 4 & 1.4 & 1.0 & 1.0 & 1.0 \\
\hline
\end{tabular}


Table S8: The calculated bimolecular rate constants (in $\mathrm{cm}^{3}$ molecule ${ }^{-1} \mathrm{~s}^{-1}$ ) for all the possible abstraction and substitution pathways involved in the DPTS $+\bullet \mathrm{OH}$ reaction in the temperatures between 200 and $300 \mathrm{~K}$ and at $0.1 \mathrm{~atm}$ pressure.

\begin{tabular}{|c|c|c|c|c|c|c|c|c|c|}
\hline $\mathbf{T}$ & $\mathbf{k}_{\mathbf{1}}$ & $\mathbf{k}_{\mathbf{2}}$ & $\mathbf{k}_{\mathbf{3}}$ & $\mathbf{k}_{\mathbf{4}}$ & $\mathbf{k}_{\mathbf{5}}$ & $\mathbf{k}_{\mathbf{6}}$ & $\mathbf{k}_{\mathbf{7}}$ & $\mathbf{k}_{\mathbf{8}}$ & $\mathbf{k}_{\mathbf{9}}$ \\
\hline 200 & $7.02 \times 10^{-12}$ & $2.24 \times 10^{-13}$ & $6.62 \times 10^{-12}$ & $9.54 \times 10^{-14}$ & $1.10 \times 10^{-15}$ & $3.78 \times 10^{-15}$ & $2.25 \times 10^{-10}$ & $7.70 \times 10^{-14}$ & $1.53 \times 10^{-14}$ \\
\hline 220 & $6.39 \times 10^{-12}$ & $1.82 \times 10^{-13}$ & $6.02 \times 10^{-12}$ & $1.04 \times 10^{-13}$ & $1.79 \times 10^{-15}$ & $5.95 \times 10^{-15}$ & $1.74 \times 10^{-10}$ & $9.64 \times 10^{-14}$ & $1.67 \times 10^{-14}$ \\
\hline 240 & $5.75 \times 10^{-12}$ & $1.58 \times 10^{-13}$ & $5.34 \times 10^{-12}$ & $1.05 \times 10^{-13}$ & $2.70 \times 10^{-15}$ & $8.65 \times 10^{-15}$ & $1.33 \times 10^{-10}$ & $1.17 \times 10^{-13}$ & $1.79 \times 10^{-14}$ \\
\hline 250 & $5.43 \times 10^{-12}$ & $1.51 \times 10^{-13}$ & $5.01 \times 10^{-12}$ & $1.03 \times 10^{-13}$ & $3.26 \times 10^{-15}$ & $1.02 \times 10^{-14}$ & $1.15 \times 10^{-10}$ & $1.29 \times 10^{-13}$ & $1.85 \times 10^{-14}$ \\
\hline 260 & $5.12 \times 10^{-12}$ & $1.45 \times 10^{-13}$ & $4.69 \times 10^{-12}$ & $1.01 \times 10^{-13}$ & $3.90 \times 10^{-15}$ & $1.19 \times 10^{-14}$ & $1.00 \times 10^{-10}$ & $1.40 \times 10^{-13}$ & $1.92 \times 10^{-14}$ \\
\hline 280 & $4.54 \times 10^{-12}$ & $1.39 \times 10^{-13}$ & $4.10 \times 10^{-12}$ & $9.47 \times 10^{-14}$ & $5.42 \times 10^{-15}$ & $1.58 \times 10^{-14}$ & $7.59 \times 10^{-11}$ & $1.65 \times 10^{-13}$ & $2.07 \times 10^{-14}$ \\
\hline 300 & $4.01 \times 10^{-12}$ & $1.36 \times 10^{-13}$ & $3.58 \times 10^{-12}$ & $8.87 \times 10^{-14}$ & $7.32 \times 10^{-15}$ & $2.05 \times 10^{-14}$ & $5.73 \times 10^{-11}$ & $1.93 \times 10^{-13}$ & $2.24 \times 10^{-14}$ \\
\hline
\end{tabular}

\begin{tabular}{|c|c|c|c|c|c|c|}
\hline $\mathbf{k}_{\mathbf{1 0}}$ & $\mathbf{k}_{\mathbf{1 1}}$ & $\mathbf{k}_{\mathbf{1 2}}$ & $\mathbf{k}_{\mathbf{1 3}}$ & $\mathbf{k}_{\mathbf{1 4}}$ & $\mathbf{k}_{\mathbf{1 5}}$ & $\mathbf{k}_{\mathbf{1 6}}$ \\
\hline $1.92 \times 10^{-13}$ & $8.30 \times 10^{-16}$ & $5.67 \times 10^{-13}$ & $1.67 \times 10^{-15}$ & $1.96 \times 10^{-12}$ & $1.26 \times 10^{-10}$ & $5.74 \times 10^{-12}$ \\
\hline $1.95 \times 10^{-13}$ & $1.31 \times 10^{-15}$ & $4.25 \times 10^{-13}$ & $2.22 \times 10^{-15}$ & $1.48 \times 10^{-12}$ & $1.04 \times 10^{-10}$ & $4.85 \times 10^{-12}$ \\
\hline $1.94 \times 10^{-13}$ & $2.02 \times 10^{-15}$ & $3.17 \times 10^{-13}$ & $2.76 \times 10^{-15}$ & $1.14 \times 10^{-12}$ & $8.45 \times 10^{-11}$ & $4.06 \times 10^{-12}$ \\
\hline $1.94 \times 10^{-13}$ & $2.46 \times 10^{-15}$ & $2.74 \times 10^{-13}$ & $3.03 \times 10^{-15}$ & $1.01 \times 10^{-12}$ & $7.56 \times 10^{-11}$ & $3.71 \times 10^{-12}$ \\
\hline $1.93 \times 10^{-13}$ & $3.00 \times 10^{-15}$ & $2.39 \times 10^{-13}$ & $3.30 \times 10^{-15}$ & $9.02 \times 10^{-13}$ & $6.74 \times 10^{-11}$ & $3.39 \times 10^{-12}$ \\
\hline $1.93 \times 10^{-13}$ & $4.30 \times 10^{-15}$ & $1.85 \times 10^{-13}$ & $3.85 \times 10^{-15}$ & $7.36 \times 10^{-13}$ & $5.31 \times 10^{-11}$ & $2.83 \times 10^{-12}$ \\
\hline $1.94 \times 10^{-12}$ & $6.02 \times 10^{-15}$ & $1.47 \times 10^{-13}$ & $4.40 \times 10^{-15}$ & $6.03 \times 10^{-13}$ & $4.16 \times 10^{-11}$ & $2.37 \times 10^{-12}$ \\
\hline
\end{tabular}


Table S9: The calculated bimolecular rate constants (in $\mathrm{cm}^{3}$ molecule ${ }^{-1} \mathrm{~s}^{-1}$ ) for all the possible abstraction and substitution pathways involved in the DPTS $+\bullet \mathrm{OH}$ reaction in the temperatures between 200 and $300 \mathrm{~K}$ and at 10 atm pressure.

\begin{tabular}{|c|c|c|c|c|c|c|c|c|c|}
\hline $\mathbf{T}$ & $\mathbf{k}_{\mathrm{TS1}}$ & $\mathbf{k}_{\mathrm{TS2} 2}$ & $\mathbf{k}_{\mathrm{TS} 3}$ & $\mathbf{k}_{\mathrm{TS} 4}$ & $2 \mathrm{k}_{\mathrm{TS} 5}$ & $\mathbf{k}_{\mathrm{TS} 6}$ & $\mathbf{k}_{\text {TS7 }}$ & $\mathbf{k}_{\text {TS8 }}$ & $\mathbf{k}_{\text {TS9 }}$ \\
\hline 200 & $9.71 \times 10^{-12}$ & $1.63 \times 10^{-13}$ & $9.76 \times 10^{-12}$ & $6.06 \times 10^{-15}$ & $1.70 \times 10^{-16}$ & $5.42 \times 10^{-16}$ & $2.37 \times 10^{-10}$ & $3.00 \times 10^{-14}$ & $4.68 \times 10^{-15}$ \\
\hline 220 & $9.19 \times 10^{-12}$ & $1.50 \times 10^{-13}$ & $9.21 \times 10^{-12}$ & $1.12 \times 10^{-14}$ & $4.63 \times 10^{-16}$ & $1.42 \times 10^{-15}$ & $1.83 \times 10^{-10}$ & $5.33 \times 10^{-14}$ & $7.65 \times 10^{-15}$ \\
\hline 240 & $8.47 \times 10^{-12}$ & $1.41 \times 10^{-13}$ & $8.44 \times 10^{-12}$ & $1.85 \times 10^{-14}$ & $1.06 \times 10^{-15}$ & $3.15 \times 10^{-15}$ & $1.38 \times 10^{-10}$ & $8.18 \times 10^{-14}$ & $1.10 \times 10^{-14}$ \\
\hline 250 & $8.05 \times 10^{-12}$ & $1.38 \times 10^{-13}$ & $7.99 \times 10^{-12}$ & $2.28 \times 10^{-14}$ & $1.52 \times 10^{-15}$ & $4.44 \times 10^{-15}$ & $1.20 \times 10^{-10}$ & $9.73 \times 10^{-14}$ & $1.26 \times 10^{-14}$ \\
\hline 260 & $7.60 \times 10^{-12}$ & $1.36 \times 10^{-13}$ & $7.52 \times 10^{-12}$ & $2.72 \times 10^{-14}$ & $2.09 \times 10^{-15}$ & $6.02 \times 10^{-15}$ & $1.04 \times 10^{-10}$ & $1.13 \times 10^{-13}$ & $1.42 \times 10^{-14}$ \\
\hline 280 & $6.69 \times 10^{-12}$ & $1.33 \times 10^{-13}$ & $6.54 \times 10^{-12}$ & $3.59 \times 10^{-14}$ & $3.61 \times 10^{-15}$ & $1.01 \times 10^{-14}$ & $7.83 \times 10^{-11}$ & $1.46 \times 10^{-13}$ & $1.73 \times 10^{-14}$ \\
\hline 300 & $5.81 \times 10^{-12}$ & $1.33 \times 10^{-13}$ & $5.62 \times 10^{-12}$ & $4.36 \times 10^{-14}$ & $5.65 \times 10^{-15}$ & $1.52 \times 10^{-14}$ & $5.90 \times 10^{-11}$ & $1.79 \times 10^{-13}$ & $2.00 \times 10^{-14}$ \\
\hline
\end{tabular}

\begin{tabular}{|c|c|c|c|c|c|c|c|}
\hline $\mathbf{k}_{\text {TS10 }}$ & $\mathbf{2 k}_{\text {TS11 }}$ & $\mathbf{k}_{\text {TS12 }}$ & $\mathbf{k}_{\mathbf{T S 1 3}}$ & $\mathbf{k}_{\mathbf{T S 1 4}}$ & $\mathbf{k}_{\text {TS15 }}$ & $\mathbf{k}_{\mathbf{T S 1 6}}$ & $\boldsymbol{k}_{\text {total }}^{\boldsymbol{a b s}+\boldsymbol{a d d}}$ \\
\hline $5.16 \times 10^{-14}$ & $3.92 \times 10^{-16}$ & $8.45 \times 10^{-14}$ & $3.09 \times 10^{-16}$ & $1.57 \times 10^{-12}$ & $9.07 \times 10^{-10}$ & $7.47 \times 10^{-12}$ & $1.17 \times 10^{-09}$ \\
\hline $7.98 \times 10^{-14}$ & $9.16 \times 10^{-16}$ & $1.03 \times 10^{-13}$ & $6.56 \times 10^{-16}$ & $1.24 \times 10^{-12}$ & $5.70 \times 10^{-10}$ & $6.20 \times 10^{-12}$ & $7.79 \times 10^{-10}$ \\
\hline $1.08 \times 10^{-13}$ & $1.66 \times 10^{-15}$ & $1.12 \times 10^{-13}$ & $1.18 \times 10^{-16}$ & $9.92 \times 10^{-13}$ & $3.59 \times 10^{-10}$ & $5.03 \times 10^{-12}$ & $5.21 \times 10^{-10}$ \\
\hline $1.21 \times 10^{-13}$ & $2.14 \times 10^{-15}$ & $1.14 \times 10^{-13}$ & $1.50 \times 10^{-15}$ & $8.91 \times 10^{-13}$ & $2.86 \times 10^{-10}$ & $4.52 \times 10^{-12}$ & $4.28 \times 10^{-10}$ \\
\hline $1.32 \times 10^{-13}$ & $2.70 \times 10^{-15}$ & $1.13 \times 10^{-13}$ & $1.85 \times 10^{-15}$ & $8.03 \times 10^{-13}$ & $2.29 \times 10^{-10}$ & $4.05 \times 10^{-12}$ & $3.54 \times 10^{-10}$ \\
\hline $1.52 \times 10^{-13}$ & $4.08 \times 10^{-15}$ & $1.08 \times 10^{-13}$ & $2.61 \times 10^{-15}$ & $6.61 \times 10^{-13}$ & $1.47 \times 10^{-10}$ & $3.27 \times 10^{-12}$ & $2.43 \times 10^{-10}$ \\
\hline $1.67 \times 10^{-13}$ & $5.84 \times 10^{-15}$ & $1.00 \times 10^{-13}$ & $3.40 \times 10^{-15}$ & $5.54 \times 10^{-13}$ & $9.68 \times 10^{-11}$ & $2.66 \times 10^{-12}$ & $1.71 \times 10^{-10}$ \\
\hline
\end{tabular}


Table S10: The calculated branching ratios for all the possible abstraction and substitution pathways involved in the DPTS + -OH reaction in the temperatures between 200 and $300 \mathrm{~K}$ and at 760 Torr pressure.

\begin{tabular}{|c|c|c|c|c|c|c|c|c|c|c|c|c|c|c|c|c|}
\hline $\mathbf{T}$ & $\mathbf{k}_{\text {TS1 }}$ & $\mathbf{k}_{\text {TS2 }}$ & $\mathbf{k}_{\text {TS3 }}$ & $\mathbf{k}_{\text {TS4 }}$ & $2 \mathbf{k}_{\mathrm{TS} 5}$ & $\mathbf{k}_{\text {TS6 }}$ & $\mathbf{k}_{\text {TS7 }}$ & $\mathbf{k}_{\text {TS8 }}$ & $\mathbf{k}_{\text {TS9 }}$ & $\mathbf{k}_{\mathrm{TS} 10}$ & $\mathbf{2 k}_{\mathrm{TS11}}$ & $\mathbf{k}_{\mathrm{TS} 12}$ & $\mathbf{k}_{\mathrm{TS13}}$ & $\mathbf{k}_{\mathrm{TS} 14}$ & $\mathbf{k}_{\mathrm{TS} 15}$ & $\mathbf{k}_{\text {TS16 }}$ \\
\hline 200 & 0.4 & 0.02 & 0.33 & 0.013 & $5.6 \times 10^{-5}$ & $1.9 \times 10^{-4}$ & 19.7 & $5.3 \times 10^{-3}$ & $9.5 \times 10^{-4}$ & 0.011 & $6.3 \times 10^{-5}$ & 0.03 & $9.1 \times 10^{-5}$ & 0.2 & 78.9 & 0.4 \\
\hline 220 & 0.6 & 0.02 & 0.54 & 0.019 & $1.7 \times 10^{-4}$ & $5.5 \times 10^{-4}$ & 23.0 & $1.1 \times 10^{-2}$ & $1.8 \times 10^{-3}$ & 0.020 & $1.6 \times 10^{-4}$ & 0.04 & $2.2 \times 10^{-4}$ & 0.2 & 74.9 & 0.6 \\
\hline 240 & 0.9 & 0.03 & 0.81 & 0.025 & $4.4 \times 10^{-4}$ & $1.4 \times 10^{-3}$ & 26.2 & $2.2 \times 10^{-2}$ & $3.2 \times 10^{-3}$ & 0.033 & $3.9 \times 10^{-4}$ & 0.05 & $4.6 \times 10^{-4}$ & 0.2 & 70.8 & 0.8 \\
\hline 250 & 1.0 & 0.03 & 0.87 & 0.026 & $6.1 \times 10^{-4}$ & $1.9 \times 10^{-3}$ & 34.9 & $2.7 \times 10^{-2}$ & $3.7 \times 10^{-3}$ & 0.038 & $5.3 \times 10^{-4}$ & 0.05 & $5.7 \times 10^{-4}$ & 0.2 & 62.0 & 0.8 \\
\hline 260 & 1.3 & 0.04 & 1.14 & 0.034 & $1.0 \times 10^{-3}$ & $3.1 \times 10^{-3}$ & 29.2 & $4.0 \times 10^{-2}$ & $5.3 \times 10^{-3}$ & 0.052 & $8.7 \times 10^{-4}$ & 0.06 & $8.7 \times 10^{-4}$ & 0.3 & 66.9 & 1.0 \\
\hline 280 & 1.7 & 0.06 & 1.51 & 0.044 & $2.2 \times 10^{-3}$ & $6.3 \times 10^{-3}$ & 32.4 & $6.9 \times 10^{-2}$ & $8.6 \times 10^{-3}$ & 0.078 & $1.8 \times 10^{-3}$ & 0.07 & $1.5 \times 10^{-3}$ & 0.3 & 62.6 & 1.2 \\
\hline 300 & 2.2 & 0.08 & 1.94 & 0.057 & $4.3 \times 10^{-3}$ & $1.2 \times 10^{-2}$ & 34.9 & $1.2 \times 10^{-1}$ & $1.4 \times 10^{-2}$ & 0.115 & $3.6 \times 10^{-3}$ & 0.08 & $2.6 \times 10^{-3}$ & 0.4 & 58.7 & 1.4 \\
\hline
\end{tabular}



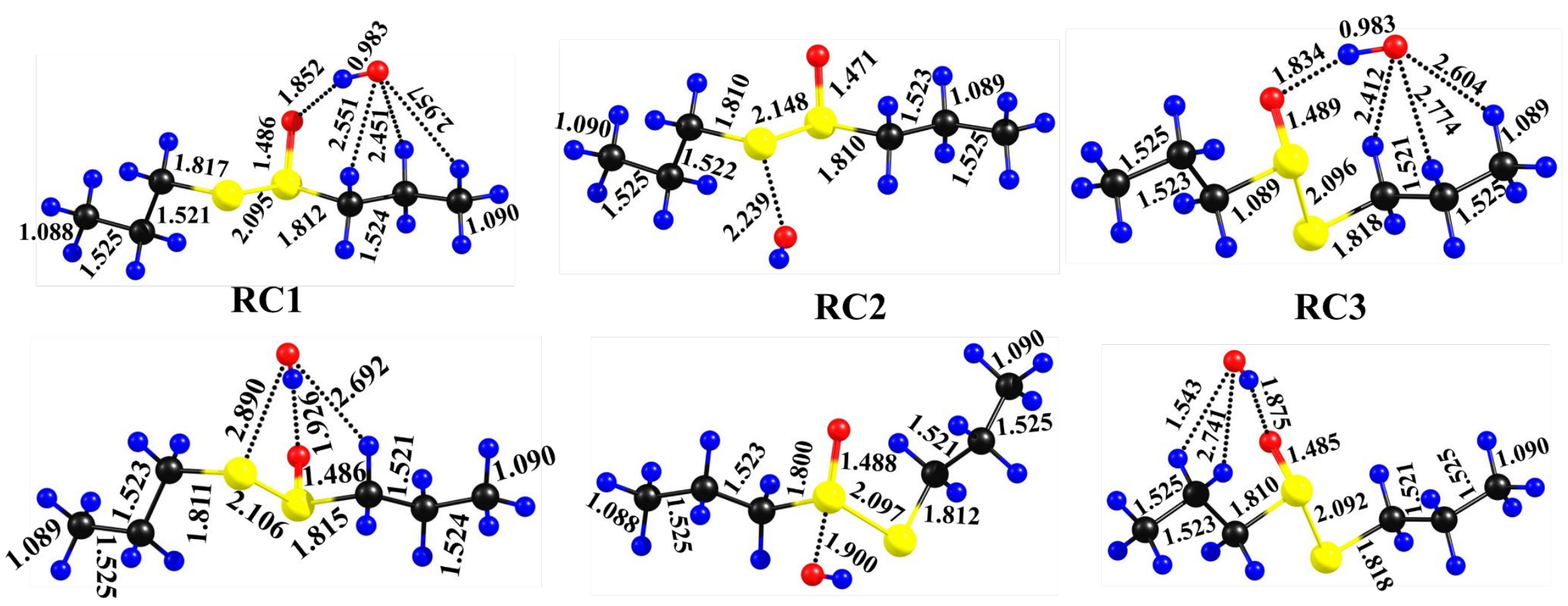

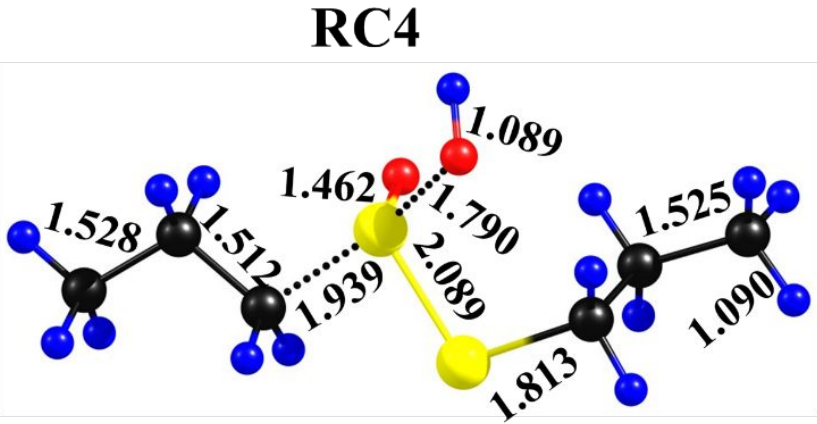

RC7

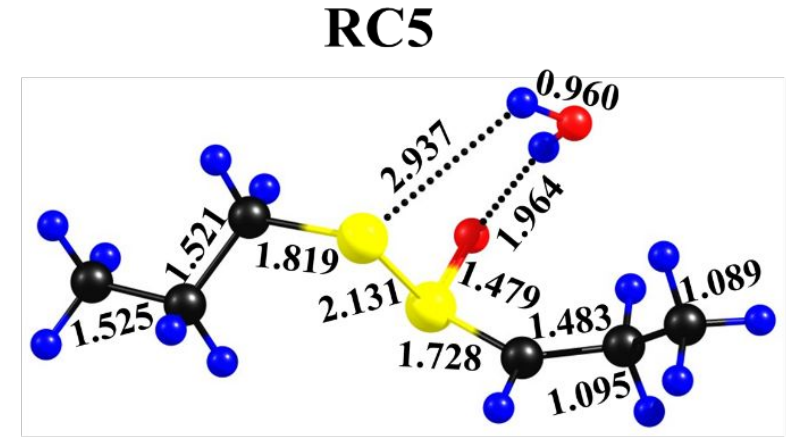

PC1

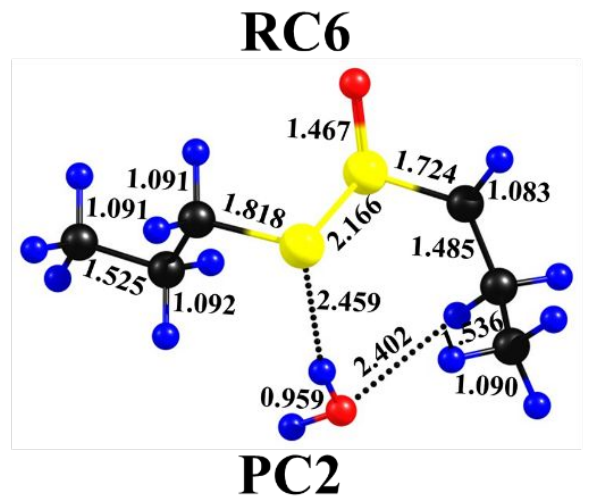




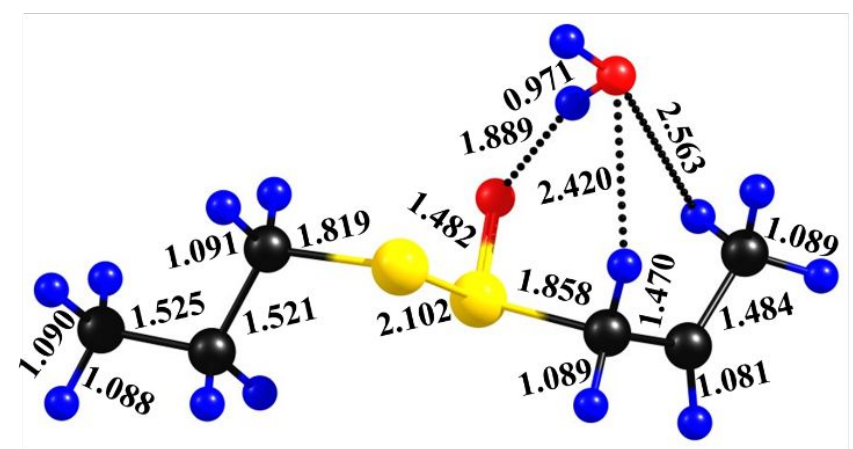

PC3

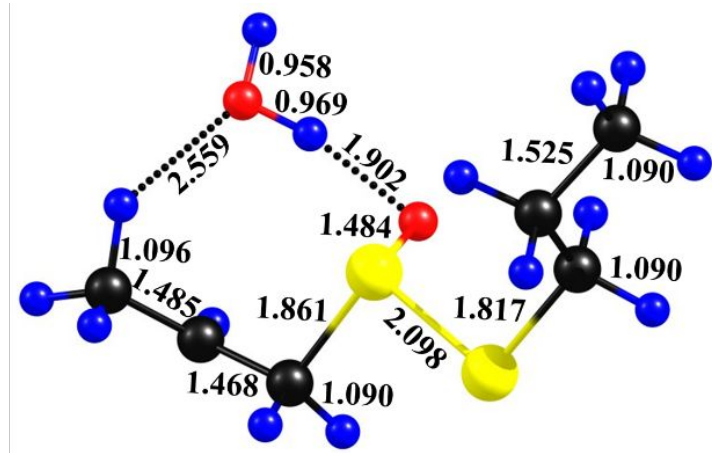

PC4

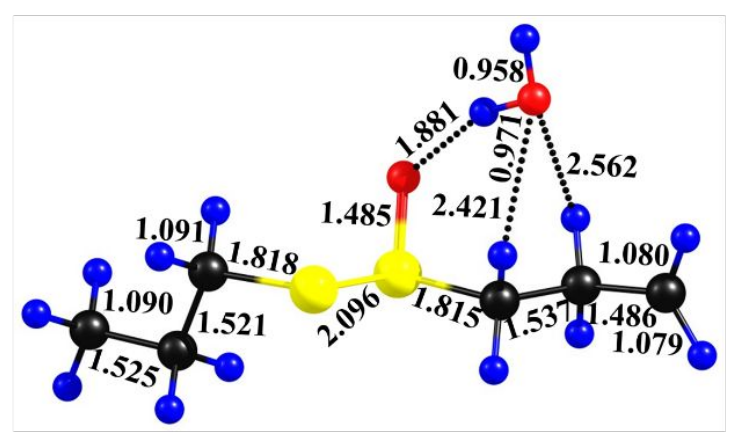

PC5

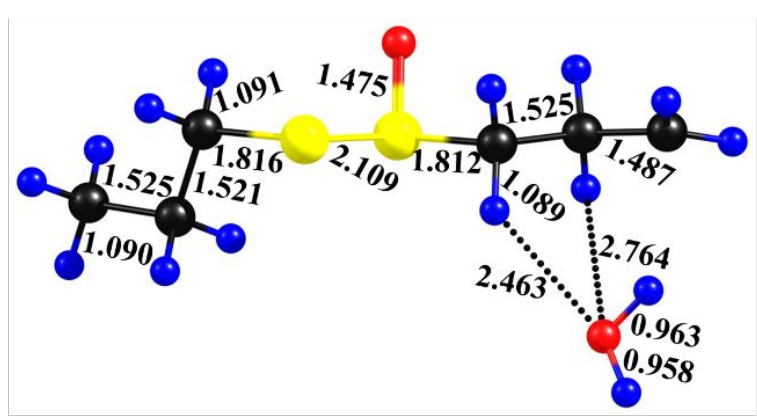

PC6

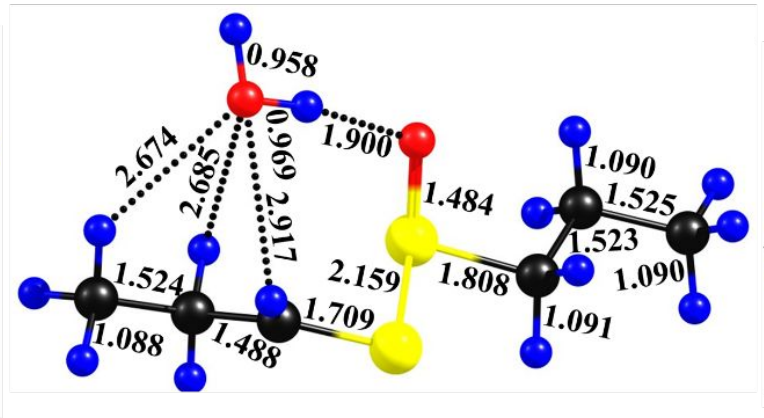

PC7

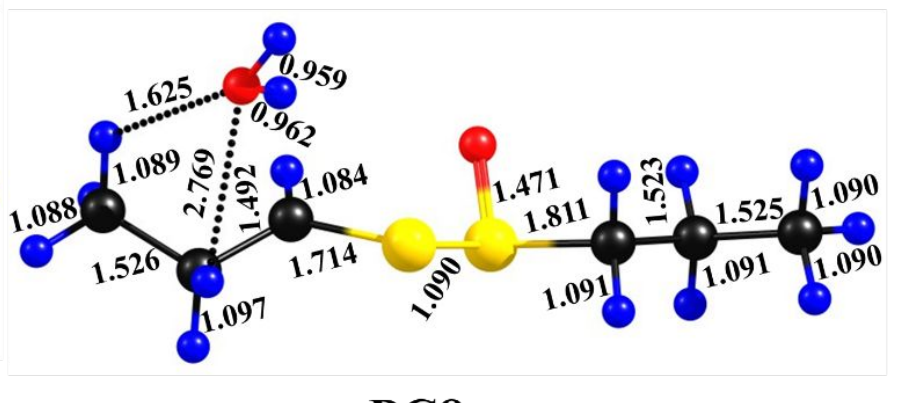

PC8

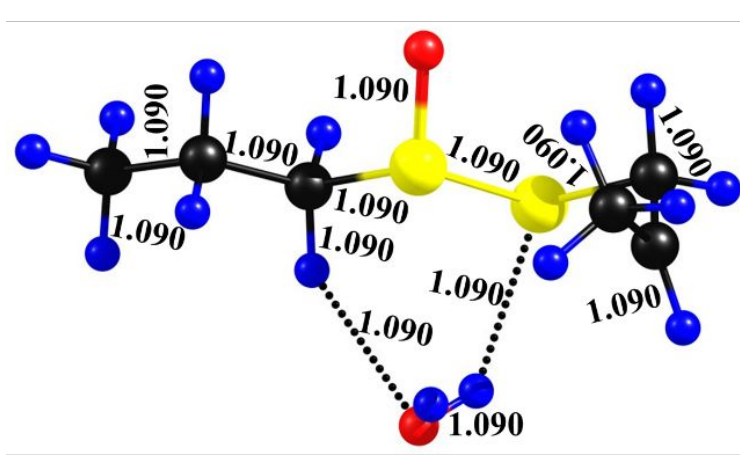

PC9

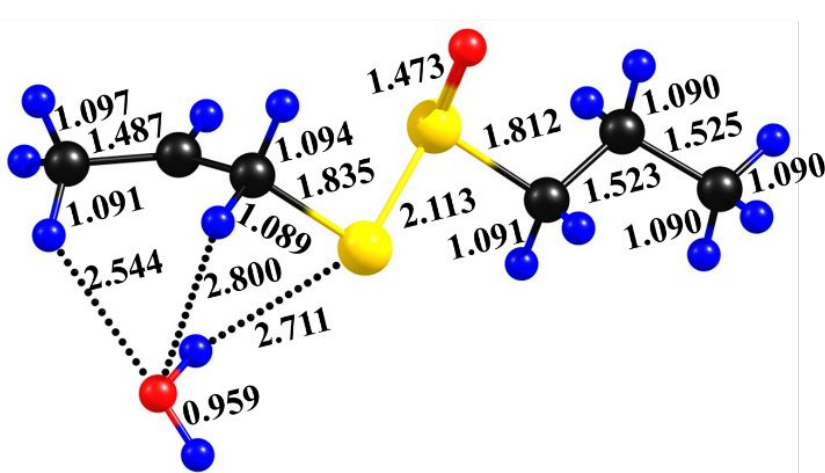

PC10

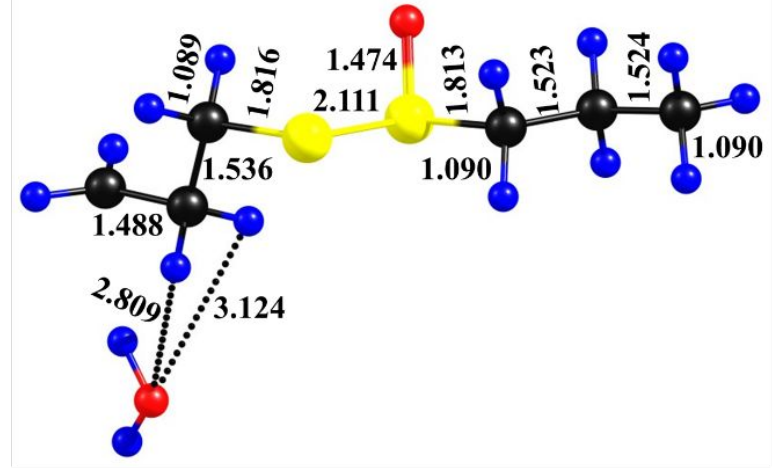

PC11 


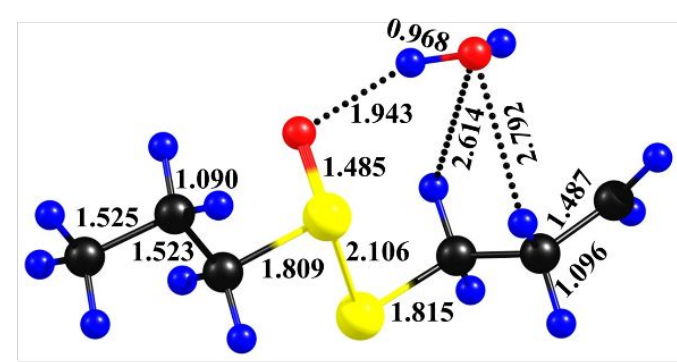

PC12
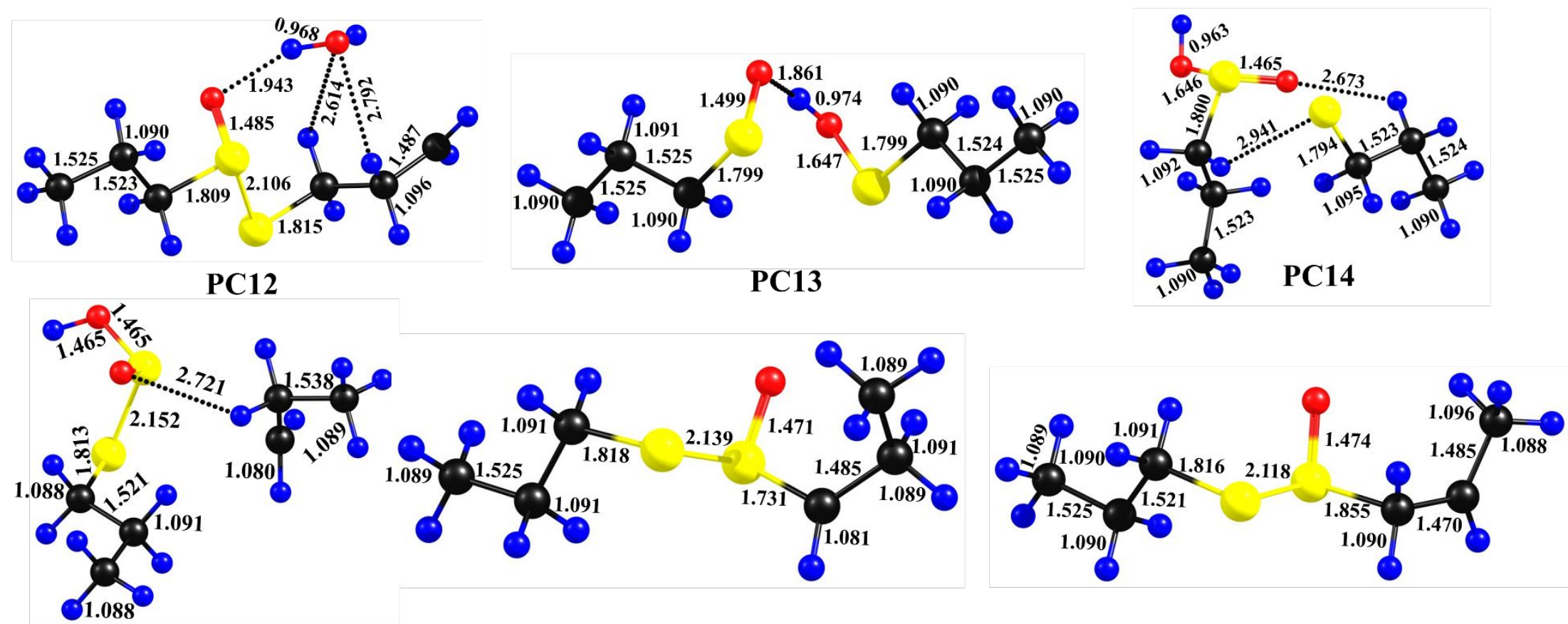

PC15

P1

P2

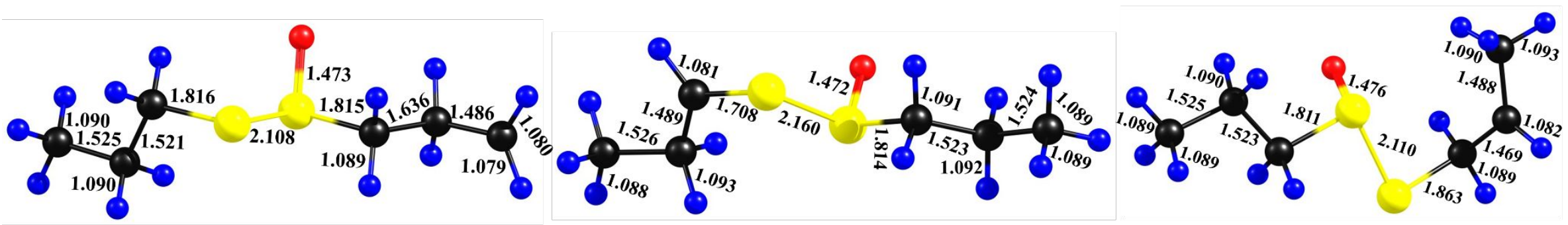

P3

P4

P5 


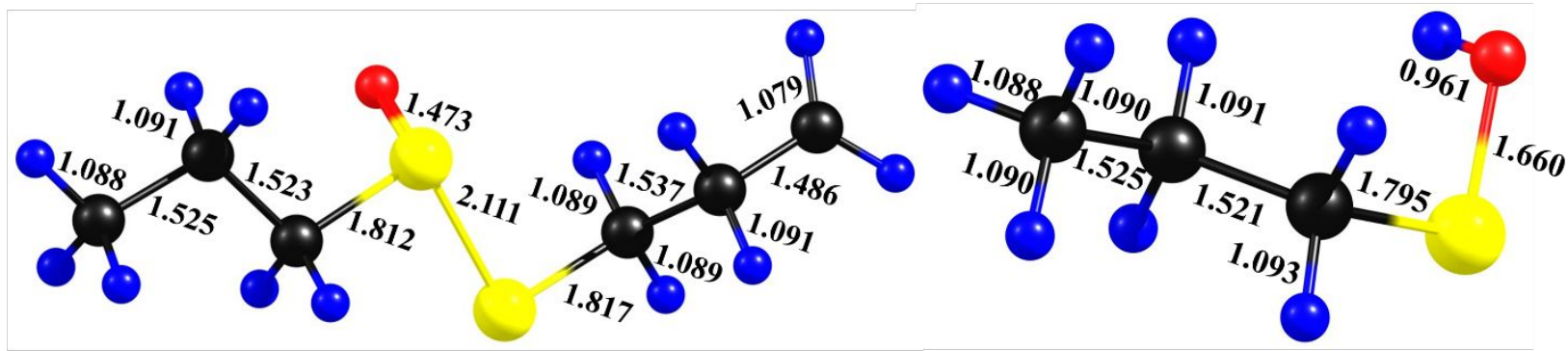

P6

P7

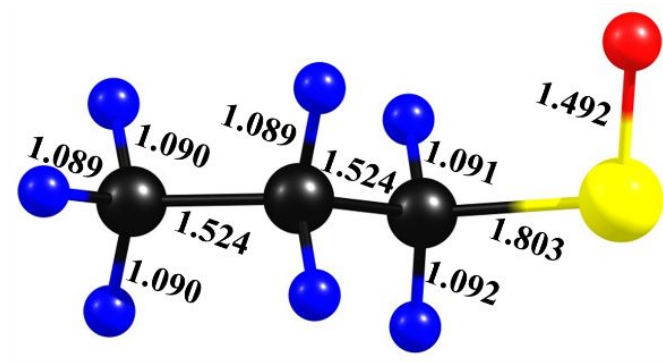

P8

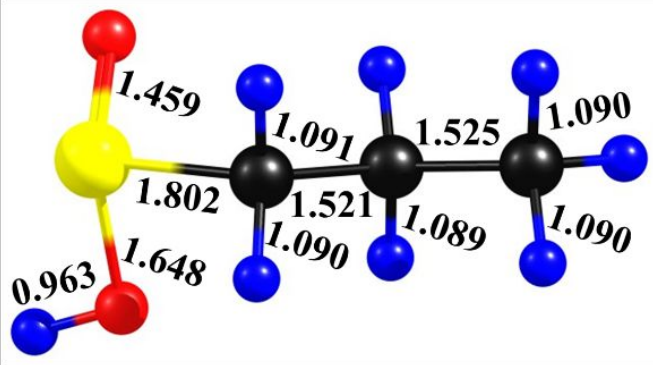

P9

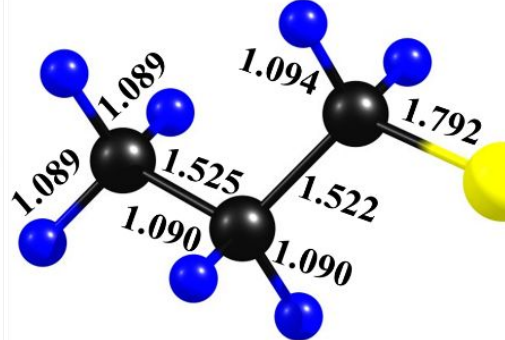

P10

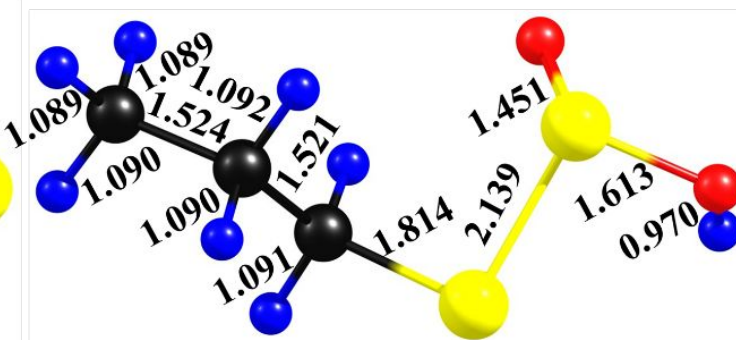

P11

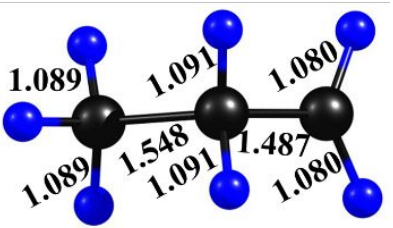

P12

Figure S1: Optimized structures of reactant complexes, product complexes, and products involved in the DPTS $+\bullet$ OH reaction system obtained at the M06-2X/6-311++G(3df,3pd) level of theory. The yellow, black, blue, and red colors represent sulfur, carbon, hydrogen, and oxygen atoms respectively. 


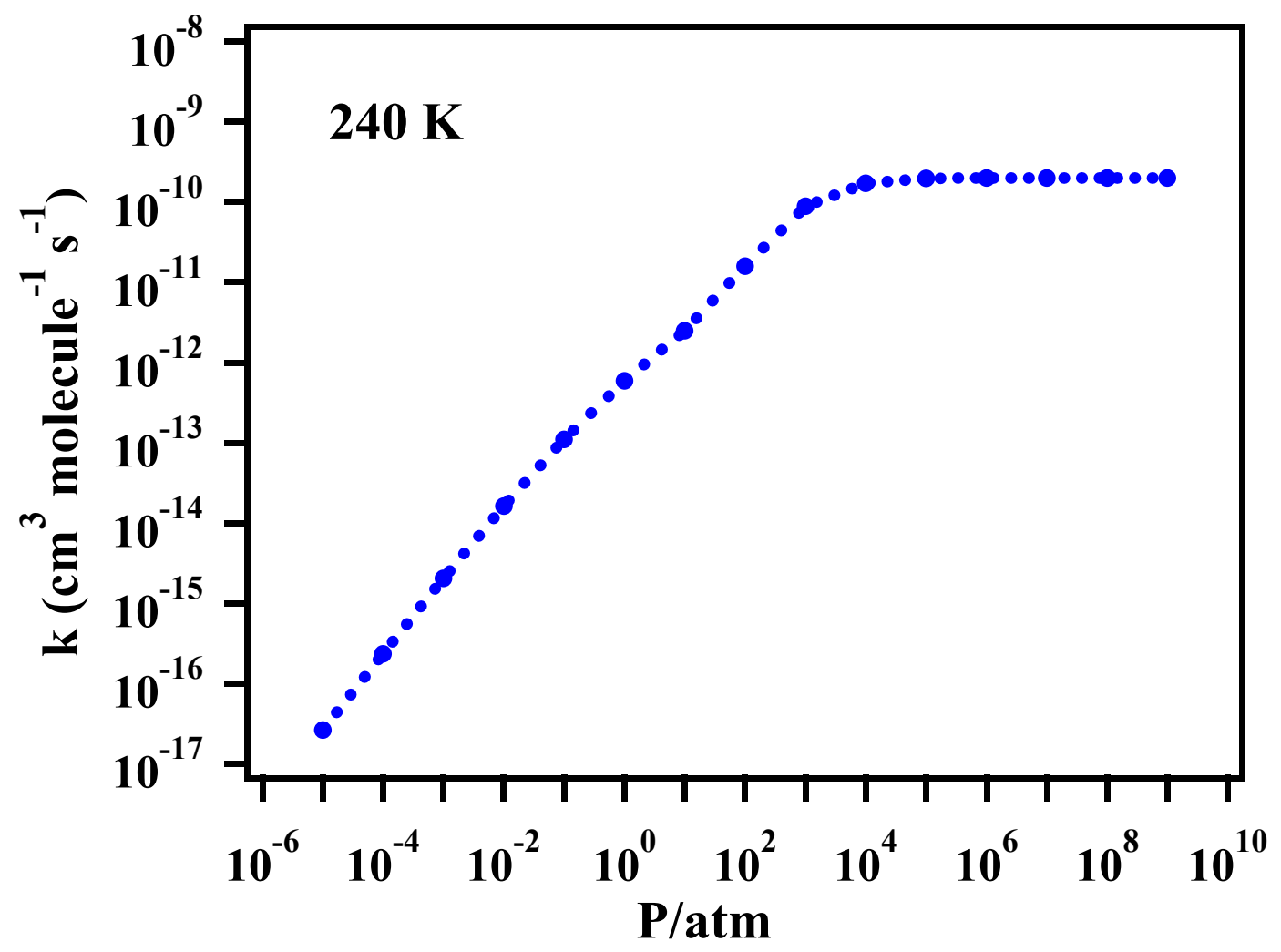

Figure S2. Rate coefficients at $240 \mathrm{~K}$ as a function of pressure for the reaction step involving association of dipropyl thiosulfinate with $\cdot O H$ leading to the formation of pre-reactive complex (RC5) for the major substitution channel (R12) involved in the DPTS $+\cdot \mathrm{OH}$ reaction. 


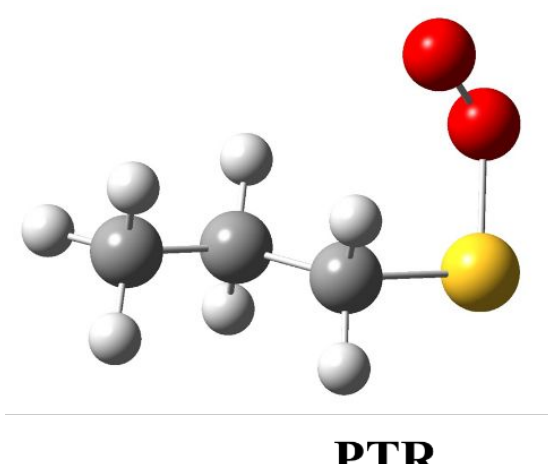

PTR

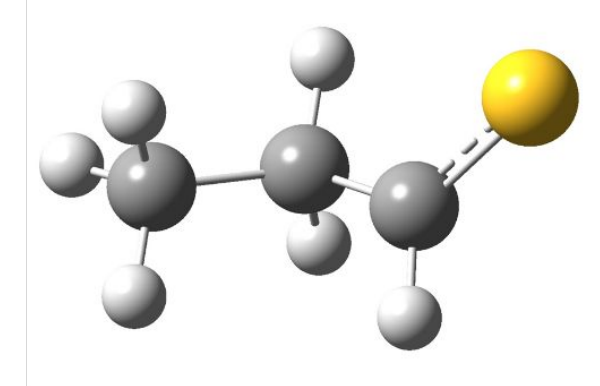

P13

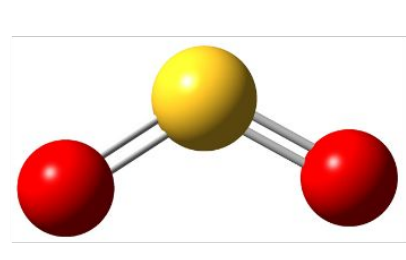

$\mathrm{SO}_{2}$

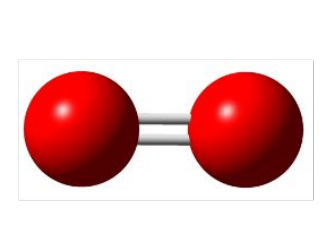

$\mathrm{O}_{2}$

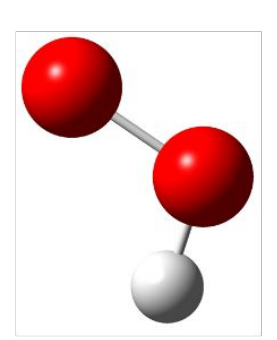

$\mathrm{HO}_{2}$

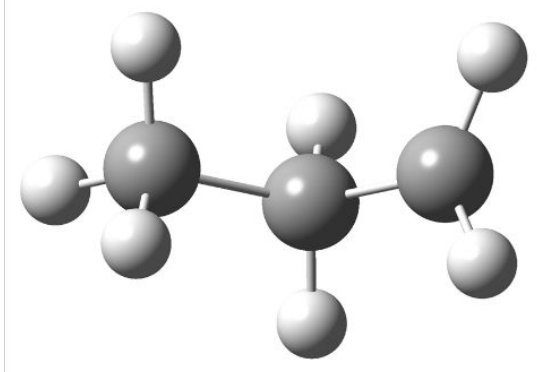

P16

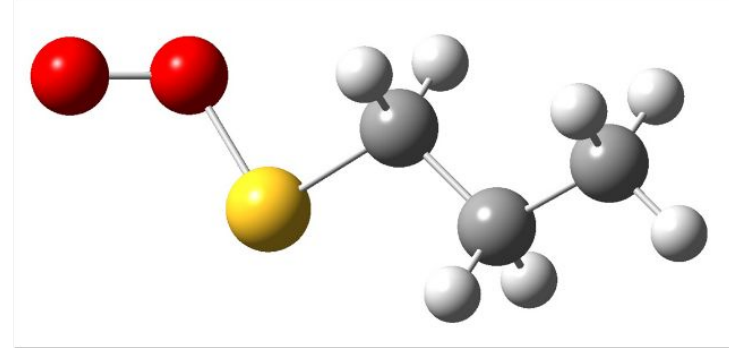

TS18

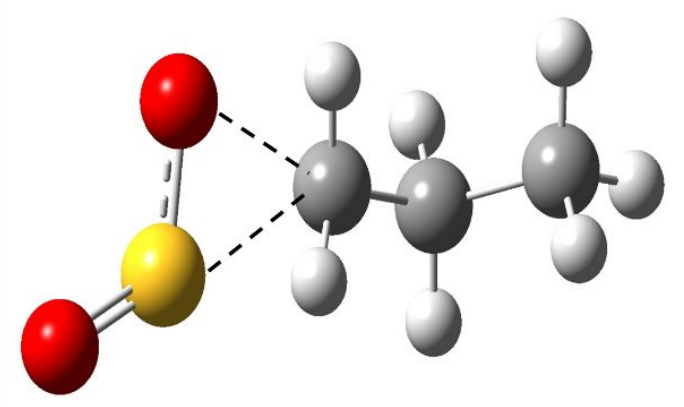

TS24

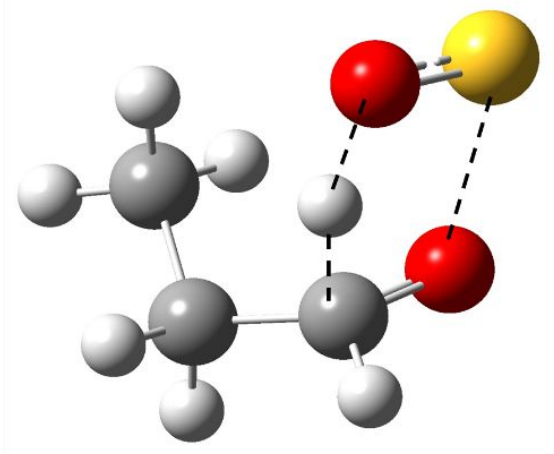

TS26

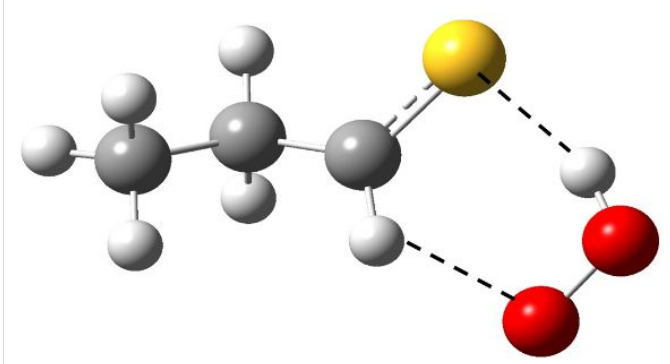

PC16

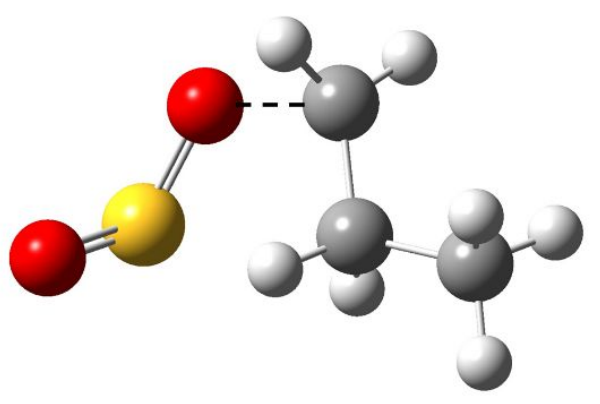

TS25

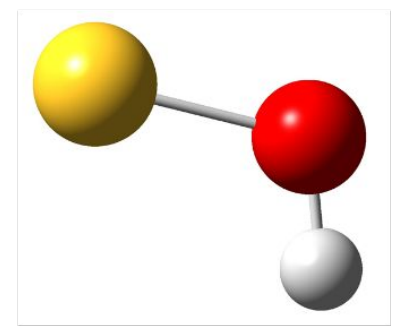

•SOH 


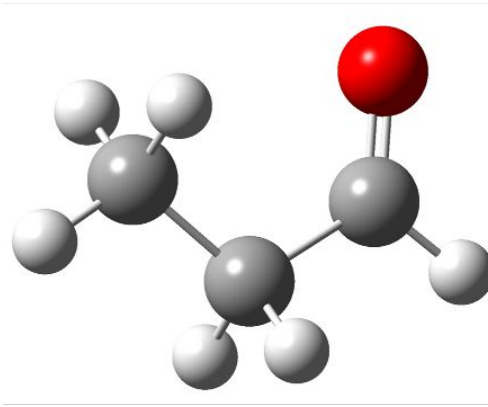

$\mathbf{P 1 7}$

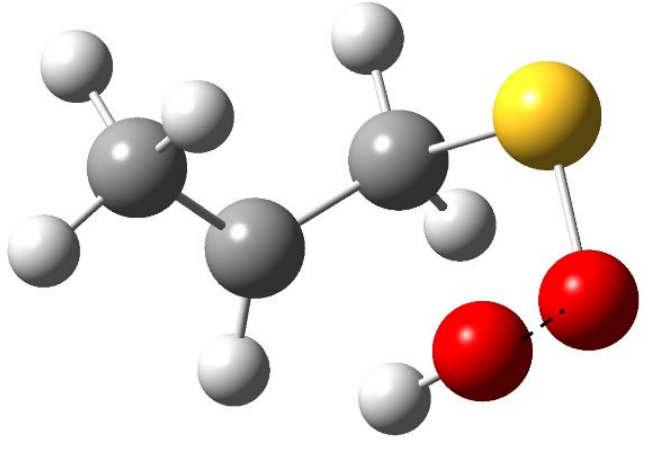

PC17

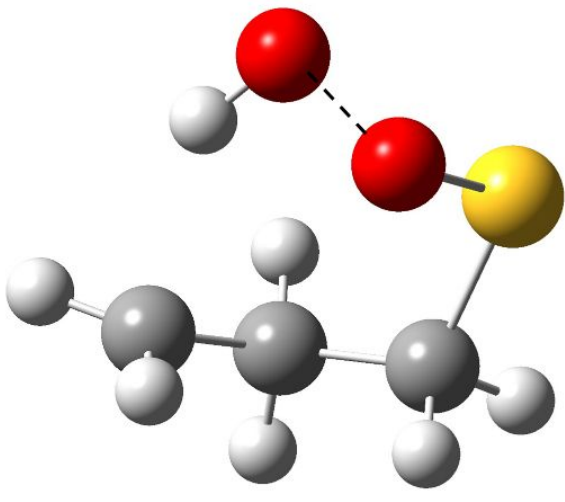

$\mathbf{P 1 5}$

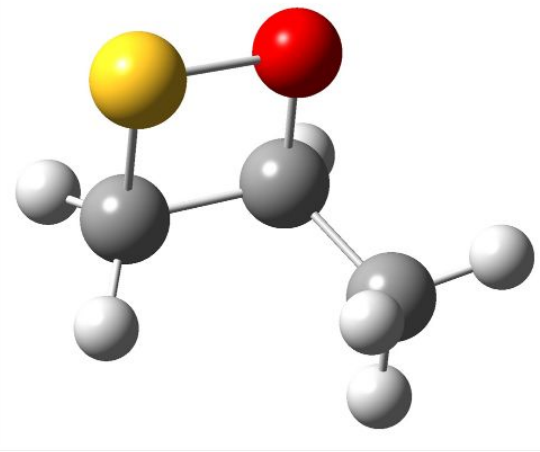

P14

Figure S3: Optimized structures of the various minima on the PES for the propanethiyl radical + atmospheric oxygen reaction system obtained at the M06-2X/6-311++G(3df,3pd) level of theory. The yellow, black, white, and red colors represent sulfur, carbon, hydrogen, and oxygen atoms, respectively. 